\author{
Universidade de São Paulo \\ Instituto de Física
}

\title{
ANÁLISE E MODELAGEM TERMODINÂMICA DE UM MODELO DE GÁS DE REDE PARA PONTES LÍQUIDAS
}

\author{
Alexandre Barros de Almeida \\ Orientador: Prof. Dr. Adriano Mesquita Alencar
}

Dissertação de Mestrado apresentada ao Instituto de Física para a obtenção do título de Mestre em Ciências

Comissão examinadora:

Prof. Dr. Adriano Mesquita Alencar (IFUSP)

Prof $^{\mathrm{a}}$. Dr ${ }^{\mathrm{a}}$. Kaline Rabelo Coutinho (IFUSP)

Prof. Dr. Roberto Fernandes Silva Andrade (UFBA)

São Paulo

2012 
FICHA CATALOGRÁFICA

Preparada pelo Serviço de Biblioteca e Informação do Instituto de Física da Universidade de São Paulo

Almeida, Alexandre Barros de

Análise e modelagem termodinâmica de um modelo de gás de rede para pontes líquidas. São Paulo, 2012.

Dissertação (Mestrado) - Universidade de São Paulo. Instituto de Física - Depto. de Física Geral

Orientador: Prof. Dr. Adriano Mesquita Alencar

Área de Concentração: Física

Unitermos: 1.Termodinâmica; 2. Mecânica estatística; 3.Respiração ruidosa.

USP/IF/SBI-100/2012 


\title{
Convenções e Lista de Símbolos
}

\author{
$\mathcal{F} \quad$ Energia Livre \\ $\mathbf{P}_{2} \quad$ Pressão do fluido 2 \\ $2 C \quad$ Curvatura de uma superfície \\ $2 h \quad$ Distância entre as placas \\ $2 h_{i} \quad$ Altura da ponte líquida \\ $2 h_{\text {min, } 1}$ Altura mínima para a formação de uma ponte líquida a partir de uma \\ gota \\ $2 h_{\min , 2}$ Altura mínima para a formação de uma ponte líquida a partir de \\ duas gotas \\ $2 h_{\text {min,c }}$ Distância crítica entre as placas da formação da ponte líquida \\ $2 h_{c} \quad$ Distância crítica entre as placas do rompimento da ponte líquida \\ $2 h_{i+1}$ Altura da ponte líquida \\ $\alpha \quad$ Número aleatório entre 0 e 1 \\ $\beta \quad \beta=\frac{1}{k_{\mathrm{B}} T}$ \\ $\Delta \mathcal{F} \quad$ Diferença de energia livre \\ $\Delta E$ Diferença de energia entre dois estados \\ $\Delta G$ Variação da energia livre de Gibbs \\ $\Delta P \quad$ Diferença de pressão \\ $\Delta U$ Diferença de energia interna \\ $\gamma_{g l} \quad$ Tensão superficial da interface gás-líquido
}


$\gamma_{g s} \quad$ Tensão superficial da interface gás-sólido

$\gamma_{l s} \quad$ Tensão superficial da interface líquido-sólido

$\gamma \quad$ Tensão superficial

$\mathcal{F}_{0,1} \quad$ Energia livre de uma única gota presa na placa

$\mathcal{F}_{b} \quad$ Energia livre da ponte líquida presa na placa

$\mathcal{H}$ Hamiltoniano do sistema

$\mathcal{N} \quad$ Número total de estados de um sistema

$\mathcal{P} \quad$ Probabilidade de obter o sistema no tempo $t$

$\mathcal{R} \quad$ Variável resumindo equações mais extensa

$\mathcal{Z} \quad$ Função de partição

$\mathcal{Z}_{i} \quad$ Função de partição da altura $2 h_{i}$

$\mathcal{Z}_{i+1} \quad$ Função de partição da altura $2 h_{i+1}$

$\mu$ Microlitros

$\mu \quad$ Potencial químico

$\Omega \quad$ Volume de líquido

$\phi_{r} \quad$ Variável resumindo equações mais extensa

$\pi \quad$ Constante matemática: $\pi=3,14159 \ldots$

$\rho \quad$ Raio da ponte líquida

$\mathrm{d}_{\mathrm{O}-\mathrm{H}}$ Tamanho da ligação entre o átomo oxigênio e hidrogênio

$\sigma \quad$ Variável resumindo equação mais extensa

$\sigma_{i}, \sigma_{j}, \sigma_{k}$ Possíveis estados acessíveis de um sistema

$\Sigma_{t} \quad$ Estado do sistema no instante de tempo $t$

$\sigma_{L J} \quad$ Distância entre duas moléculas de água na qual o potencial de LennardJones é nulo

$\tau \quad$ Uma unidade de tempo de Monte Carlo 
$\mathbf{P}_{1} \quad$ Pressão do fluido 1

$\Theta \quad$ Angulo de contato

$\tilde{E}(k, \phi)$ Variável resumindo equações mais extensa

$\tilde{F}(k, \phi)$ Variável resumindo equações mais extensa

$\varepsilon_{L J}$ Profundidade do poço do potencial de Lennard-Jones da interação entre duas moléculas de água

$\xi \quad$ Representação de reta normal

A Área

$a \quad a=R_{P} / R_{B}$

$A_{B} \quad$ Área da interface líquido-sólido

$A_{R} \quad$ Área da interface gás-líquido

$A_{T} \quad$ Área da interface gás-sólido

$B \quad$ Parâmetro de ajuste

b Variável resumindo equações mais extensa

$c_{i} \quad$ Número de ocupação

$d A \quad$ Variação infinitesimal da área de uma interface

$d r \quad$ Variação infinitesimal do raio

$d V_{1} \quad$ Variação infinitesimal do volume do meio 1

$d V_{2} \quad$ Variação infinitesimal do volume do meio 2

$d W \quad$ Variação infinitesimal do trabalho

e Carga do eletron

$E_{1} \quad$ Energia de um sistema com duas partículas interagindo na distância 1

$E_{m} \quad$ Hamiltoniano do sítio $m$ de uma rede

$E_{\text {cin }} \quad$ Energia cinética 
$E_{\text {interf }}$ Energia interfacial

$E_{\text {pot }}$ Energia potencial gravitacional

$E_{\text {tot }}$ Energia total

$E_{\sigma_{j}} \quad$ Energia do estado $\sigma_{j}$

$E_{\sqrt{2}}$ Energia de um sistema com duas partículas interagindo na distância $\sqrt{2}$

$E_{\sqrt{3}}$ Energia de um sistema com duas partículas interagindo na distância $\sqrt{3}$

$E_{\text {sep }}$ Energia de um sistema com duas partículas de líquido separadas por uma partícula de gás

$F(k, \phi), E(k, \phi)$ Integrais elípticas de Legendre

$g \quad$ Partículas de gás

$h_{P} \quad$ Constante de Planck

$i, j, k, m$ Índices

$J \quad$ Energia de troca

$J_{i j}^{1} \quad$ Energia de ligação de duas partículas na distância 1 , onde $i, j=s, l, g$

$J_{i j}^{2} \quad$ Energia de ligação de duas partículas na distância $\sqrt{2}$, onde $i, j=$ $s, l, g$

$J_{i j}^{3} \quad$ Energia de ligação de duas partículas na distância $\sqrt{3}$, onde $i, j=$ $s, l, g$

$J_{S} \quad$ Energia da ligação da molécula de água com a amostra

$J_{T} \quad$ Energia da ligação da molécula de água com a ponta do MFA

$J_{i j} \quad$ Energia de ligação de duas partículas, onde $i, j=s, l, g$

$k \quad$ Variável resumindo equações mais extensa

$k_{\mathrm{B}} \quad$ Constante de Boltzmann

L $\quad$ Lado de uma rede cúbica 
l Partículas de líquido

$M \quad$ Representação de um ponto numa superfície

$N \quad$ Número de partículas no sistema

n Número de mols

nn Primeiros vizinhos

$p \quad$ Probabilidade condicional

$P_{0} \quad$ Pressão normal de vapor

$P_{i} \quad$ Probabilidade de se obter uma diferença de energia potencial $\Delta U$ no espaço de configurações do da altura $2 h_{i}$

$P_{S} \quad$ Pressão de vapor sobre a superfície de um fluido

$P_{1} \quad$ Probabilidade de obter a energia $E_{1}$

$P_{\sqrt{2}} \quad$ Probabilidade de obter a energia $E_{\sqrt{2}}$

$P_{\sqrt{3}} \quad$ Probabilidade de obter a energia $E_{\sqrt{3}}$

$P_{i+1} \quad$ Probabilidade de obter uma diferença de energia potencial no espaço de configurações da altura $2 h_{i+1}$

$P_{\text {sep }} \quad$ Probabilidade de obter a energia $E_{\text {sep }}$

$q_{H} \quad$ Carga no átomo hidrogênio

$q_{O} \quad$ Carga no átomo oxigênio

$R \quad$ Constante universal dos gases

$r, r^{\prime} \quad$ Raio

$R_{0} \quad$ Raio da gota aproximada por uma esfera

$R_{0}^{\prime} \quad$ Raio da gota aproximada por uma esfera com metade do volume do caso com somente uma gota

$R_{1} \quad 1^{o}$ raio principal da curvatura de uma superfície

$R_{2} \quad 2^{o}$ raio principal da curvatura de uma superfície

$R_{B} \quad$ Raio da base da ponte líquida 
$R_{P} \quad$ Raio do "pescoço" da ponte líquida

$S \quad$ Entropia

$s \quad$ Partículas de sólido

$S_{a} \quad$ Sítios da superfície da amostra

$S_{p} \quad$ Sítios da superfície da ponta do MFA

T Temperatura

$t \quad$ Tempo

$U \quad$ Energia interna

u Variável resumindo equações mais extensa

$u_{0} \quad$ Energia interna por partícula de uma partícula de líquido cercada por líquidos

$U_{i} \quad$ Energia interna da altura $2 h_{i}$

$U_{i+1} \quad$ Energia interna da altura $2 h_{i+1}$

$V \quad$ Volume do sistema

$v \quad$ Variável resumindo equação mais extensa

$v_{\text {eff }} \quad$ Volume efetivo de um sítio da rede

$w_{j k} \quad$ Probabilidade de transição entre os estados $\sigma_{j}$ e $\sigma_{k}$

p Coordenadas de momento

r Coordenadas de posição

$\mathbf{p}_{E} \quad$ Pressão externa

$\mathbf{p}_{I} \quad$ Pressão interna

$\mathbf{p}_{T H} \quad$ Pressão de ruptura da membrana líquida

H-Ô-H Ângulo formado entre as ligações covalentes na molécula de água

MC Monte Carlo

MFA Microscópio de força atômica 
SPC Modelo da molécula de $\mathrm{H}_{2} \mathrm{O}$

TIP3P Modelo da molécula de $\mathrm{H}_{2} \mathrm{O}$ 


\section{Resumo}

Nesta dissertação, estudou-se um modelo tipo gás de rede em três dimensões para simular sistemas líquidos macroscópicos. Aplicou-se o modelo para o estudo das energias e forças envolvidas durante o processo de formação e ruptura de pontes líquidas entre duas placas planas. Esse estudo foi motivado por processos fisiológicos que acontecem no interior dos pulmões dos mamíferos. Além disso, foi feito um estudo das propriedades termodinâmicas do modelo. Com relação a aplicação fisiológica, observou-se que, no processo da formação da ponte líquida, a energia livre da ponte líquida é menor que a energia livre da gota, para diferentes sis-

temas líquidos. Com este resultado, fez a hipótese de que parte dessa energia é dissipada na forma de energia acústica. A emissão do som também deve ocorrer na ruptura da ponte líquida. Comparando a energia livre no processo de formação e ruptura da ponte líquida observou-se uma curva de histerese. Também foi verificado que para sistemas pequenos, a ponte líquida no modelo computacional se forma antes da previsão analítica. Para a análise termodinâmica, o modelo foi simplificado removendo as placas planas. Foi estudado o caso mais simples desse modelo que continha apenas duas partículas de líquido. Neste caso, calculouse o calor específico e a energia interna numericamente, e esses resultados foram comparados com cálculos analíticos, validando o modelo numérico. Posteriormente, realizou-se um estudo da transição de fase desse sistema. Em seguida, a energia livre e a força da ponte líquida sobre as placas foram estudadas para diferentes temperaturas utilizando duas metodologias. Na primeira metodologia a entropia foi desprezada, na segunda metodologia, foi utilizando o método "Overlapping Distribution" que considera a entropia do sistema. Foi concluído que a entropia tem um efeito muito pequeno nas condições estudadas. O modelo é viável para 
a modelagem de fluidos a nível macroscópico e que portanto pode ser utilizado para quantificar não só as forças internas de estruturas pulmonares como também avaliar as energias liberadas após o processo de ruptura ou formação dessas pontes. 


\begin{abstract}
This work studied a three dimension lattice gas model to simulate macroscopic liquid systems. We used the model to study the energy and the forces involved during the process of liquid bridge formation and rupture between two parallel planes. The motivarion of this study was a physiological processes which occur inside the mammals lungs. Furthermore, a study was made to elucidate thermodynamic properties of the model. Concerning to physiological application, it was observed that the free energy of liquid bridge is smaller than the free energy of the droplet, for different liquid systems. With this observation, was proposed that this energy is dissipated as an acoustic energy. This sound should also exist in the rupture of liquid bridge. Comparing the free energy of liquid bridge in the formation and rupture process was observed a hysteresis curve. It was also found an effect of finite size in the formation of small size of the liquid bridge. In the numerical model, the liquid bridge is always formed earlier than expected from the analytical model. In the thermodynamic study, the model was simplified removing both parallel planes. First, the simplest case of this model was studied, only two liquid particles in a large gas lattice. In this case, the specific heat and internal energy was numerically studied and the results was compared with analytical calculation. Subsequently, we carried out a study of the phase transition of this system. Then, the free energy and the force generated between two parallel planes due the presence of the liquid bridge. This studied was performed using two different temperatures and two distinct methods. In the first method the entropy was neglected, and in the second method not. The second method was the "Overlapping Distribution". It was concluded that the entropy has a very small effect in the studied conditions. The model is viable for modeling fluids at macroscopic level and therefore can be used to quantify not only
\end{abstract}


the internal forces of the lung structures, but evaluate the energies released after the rupture process of the formation of these bridges. 


\section{Agradecimentos}

De maneria especial, agradeço:

- ao professor Adriano M. Alencar pelo apoio em realizar este trabalho e pelas discussões e motivações em desenvolver novas ideias;

- à minha família que tem me ajudado desde sempre nas dificuldades;

- Aos meus amigos do grupo: Camila Cristina Won Ancken, Diana M. Martínez, Eduardo Redoschi, Mac Gayver S. Castro, Marcel Philippi Dorta, Mariana Sacrini Ayres Ferraz, Marcia Zotti Justo Ferreira, Danilo Furlan Kaid, Victor Mendizabal Coelho e Wagner Shin Nishitani pelos momentos de diversão e risadas e pelas sugestões na realização deste trabalho.

- às agências de fomento: Fundação de Amparo à Pesquisa do Estado de São Paulo (FAPESP) e Coordenação de Aperfeiçoamento de Pessoal de Nível Superior (CAPES) pela bolsa, e ao Conselho Nacional de Desenvolvimento Científico e Tecnológico (CNPq) pelo apoio à pesquisa. 


\section{Sumário}

1 Introdução 1

2 Modelos Analíticos e Numéricos da Ponte Líquida 11

2.1 A Equação de Young-Laplace . . . . . . . . . . . . . . . . . . 11

2.2 Soluções Analíticas . . . . . . . . . . . . . . . . . . . . . . . 14

2.3 Modelo de Gás de Rede . . . . . . . . . . . . . . . . . . . . . 22

2.4 Método Monte Carlo com Algoritmo de Metropolis . . . . . . 26

2.5 Dinâmica de Kawasaki . . . . . . . . . . . . . . . . . . . . . . 29

2.6 Energia Livre: Método "Overlapping Distribution" . . . . . . . 30

3 Resultados para o Modelo Macroscópico da Ponte Líquida 35

3.1 Estudo da Formação . . . . . . . . . . . . . . . . . . 36

3.1.1 Diferença de Energia Livre de uma Gota e da Ponte Líquida . . . . . . . . . . . . . . 36

3.1.2 O Efeito da Interação de Longo Alcance . . . . . . . . 37

3.1.3 Histerese na Energia Livre . . . . . . . . . . . . . . . 42

3.1.4 Força Exercida na Placa . . . . . . . . . . . . . . 43

3.2 Analise Termodinâmica do Modelo . . . . . . . . . . . . . . 45

3.2.1 Sistema com Duas Partículas do Líquido . . . . . . . . 46

3.2.2 Estudo do Efeito da Escala . . . . . . . . . . . . 51

3.2.3 Estudo da Transição de Fase do Líquido . . . . . . . . 53

3.2.4 Calculo da Energia Livre . . . . . . . . . . . . . . 55

4 Conclusão $\quad 65$

A A Equação de Kelvin $\quad 69$

B Artigo Submetido $\quad 71$

Referências Bibliográficas $\quad 93$ 


\section{Capítulo 1}

\section{Introdução}

Suponha que uma pequena quantidade de um fluido seja colocado sobre uma placa plana, o mesmo formará uma gota sobre a placa, com um ângulo de contato. Agora, uma segunda placa, paralela à primeira, é aproximada até encostar na superfície dessa gota. Existe uma distância mínima entre as placas na qual haverá a formação de uma ponte líquida unindo as duas placas. Nesta dissertação, desenvolveu-se um modelo computacional tipo gás de rede para estudar essa ponte líquida e algumas propriedades termodinâmicas desse fluido. Nesse contexto, o estudo das pontes líquidas é importante para o processo industrial de sinterização ${ }^{1}$ de fase líquida, fisiologia pulmonar e microscopia de força atômica (MFA). Antes de introduzir esse modelo computacional, será comentado um pouco mais sobre essas três motivações.

A primeira motivação apresentada está no processo de sinterização de fase líquida. Nesse processo, pontes líquidas surgem entres os grãos do pó

\footnotetext{
${ }^{1}$ A sinterização é um processo industrial para produção de cerâmicas e materiais metálicos a partir do pó de uma substância colocada em uma temperatura próxima a de fusão [1]. Nessa temperatura, os grãos começam a se fundir dando origem às cerâmicas e materiais metálicos.
} 
da substância, gerando uma força capilar que aumenta a velocidade de sinterização, organiza melhor o material e, consequentemente, gera um material mais resistente $[2,3]$.

Com relação à fisiologia pulmonar, que foi a motivação original desse trabalho, a superfície interna do pulmão é coberta por um fluido composto por água, biomoléculas e um outro fluido complexo denominado surfactante pulmonar [4-7]. O surfactante é responsável por regular a tensão superficial na interface ar-líquido do pulmão. Alterações na composição do surfactante pode diminuir a tensão superficial que, por sua vez, pode levar à formação de pontes líquidas nas vias aéreas respiratórias, dificultando, assim, as trocas gasosas nos sacos alveolares [8, 9]. Durante a inspiração, as pontes líquidas que estão bloqueando as vias aéreas, podem se romper devido às instabilidades mecânicas [10-12] e emitir um ruído discreto denominado de ruído de crepitação [13-15], que pode ser facilmente auscultado com um estetoscópio.

${ }^{2}$ Para compreender o ruído de crepitação [16], considere-se um tubo com ar dividido ao meio por uma membrana líquida. Hipoteticamente, um dos lados do tubo estaria conectado com a traqueia, aberto para a atmosfera, e o outro estaria conectado à parte interna do pulmão, o qual tem sua pressão continuamente reduzida devido à expansão da caixa torácica no processo de inspiração. No processo de inspiração, o gradiente de pressão é continuamente incrementado até que ocorra a ruptura da membrana. No instante que antecede a quebra da membrana no limiar de ruptura, as pressões se

\footnotetext{
${ }^{2} \mathrm{O}$ texto em itálico modificado da Ref. [16].
} 
equilibram seguindo a equação:

$$
\mathbf{p}_{I}+\mathbf{p}_{E}+\mathbf{p}_{T H}=0
$$

onde $\mathbf{p}_{I}$ é a pressão interna, $\mathbf{p}_{E}$ a pressão externa, respectivamente do lado esquerdo e direito na Fig. 1.1, e $\mathbf{p}_{T H}$ é a pressão de ruptura da membrana líquida. Essa pressão de ruptura será determinada pelas propriedades do
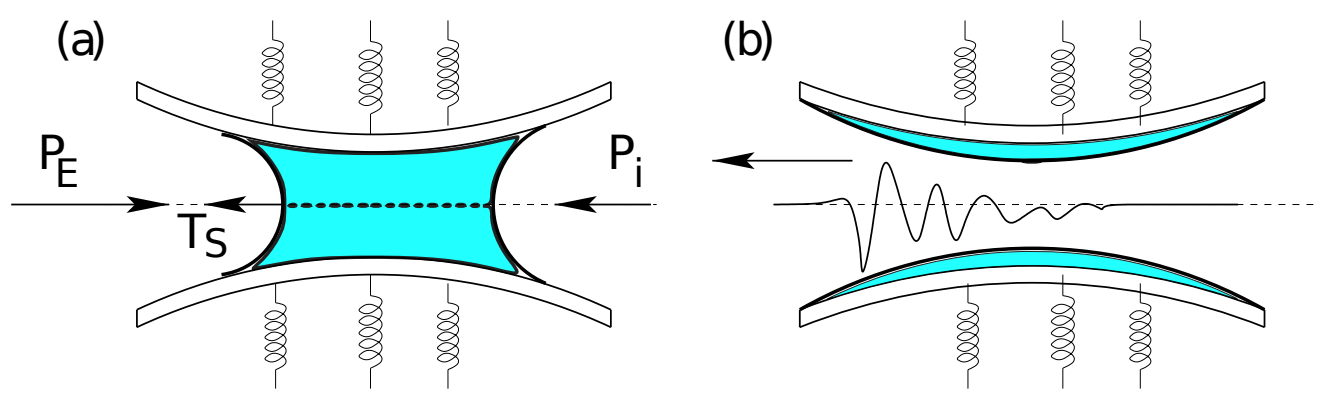

Figura 1.1: Figura adaptada da Ref. [16].(a) Tubo com uma ponte formada por uma membrana líquida submetida a uma pressão externa maior que a interna, $p_{e}$ e $p_{i}$ respectivamente. O equilíbrio das forças é feito pela tensão superficial $\gamma$; (b) gráfico esquemático da geração de um ruido de crepitação logo após a ruptura da membrana, a seta indica o sentido da propagação da onda acústica.

líquido que compõem a membrana, logo, dependera da tensão superficial da mesma.

A terceira motivação dessa dissertação possui aplicações na microscopia de força atômica (MFA) e foi onde o maior números de modelos matemáticos, análogos ao discutido nesta dissertação, foram encontrados. Essa técnica tem sido utilizada para estudar as pontes líquidas na escala nanométrica. Durante os experimentos com MFA em um ambiente que contenha umidade, uma ponte líquida será formada instantes antes da ponta do MFA tocar a 
superfície em estudo[17-19]. Na Figura 1.2, pode-se observar uma imagem obtida com um microscópio eletrônico de varredura de uma ponte líquida formada na ponta do MFA.

Essa ponte líquida nanométrica produz uma força capilar na ponta do MFA que altera a resolução das imagens obtidas [20]. Além disso, tem-se utilizado o MFA para estudar a validade da lei de Kelvin (Apêndice A), que relaciona a mudança na pressão de vapor devido à presença de uma interface vapor-líquido para escalas nanométricas [21, 22]. A equação de Kelvin pressupõe que o raio médio das moléculas seja muito menor do que a curvatura da superfície, o que não acontece na escala nanométrica.

A influência da ponte líquida na resolução do MFA e a questão da validade da lei de Kelvin motivaram a elaboração de modelos computacionais para estudar pontes líquidas na escala nanométrica. Tem-se utilizado um modelo estatístico tipo gás de rede e dinâmica molecular para estudar essas pontes líquidas.
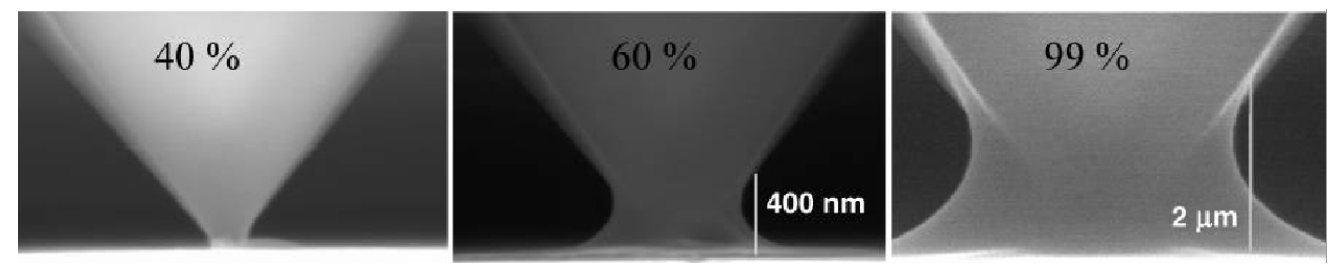

Figura 1.2: Imagens de pontes líquidas formadas na ponta do MFA, por Weeks e colaboradores [19]. Pode-se observar a ponte líquida formada para três valores de umidade relativa: 40\%, 60\% e 99\%. Na imagem do meio têmse uma ponte líquida de 400nm e na imagem da direita uma ponte líquida de $2 \mu \mathrm{m}$. Essas imagens foram feitas por um microscópio eletrônico de varredura acoplado ao MFA [19].

No primeiro modelo estatístico para o estudo da ponte líquida na ponta 
do MFA, proposto por Jang e colaboradores [23], foi utilizada uma rede em duas dimensões com condição periódica de contorno. Cada sítio da rede que esta ocupado por uma molécula de água, interage com os primeiros vizinhos da rede $n n$. Nesse estudo, o ensemble utilizado foi o grande canônico e as configurações do sistema foram geradas com o método de Monte Carlo com amostragem de Metropolis. Nesse modelo foi utilizado um Hamiltoniano do tipo:

$$
\mathcal{H}=-J \sum_{i, j=n n} c_{i} c_{j}-J_{T} \sum_{i=S_{p}} c_{i}-J_{S} \sum_{i=S_{a}} c_{i}-\mu N
$$

em que $c_{i}$ é o número de ocupação (0 ou 1) do i-ézimo elemento, $J$ é a energia de troca e neste caso corresponde à energia de ligação de hidrogênio entre duas moléculas de água, $J_{T}$ é a energia da ligação da molécula de água com a ponta do MFA, $J_{S}$ é a energia da ligação da molécula de água com a amostra, $\mu$ é o potencial químico, $N$ é o número de partículas no sistema, que neste caso uma partícula é o mesmo que uma molécula de água, $S_{p}$ são os sítios da superfície da ponta do MFA e $S_{a}$ são os sítios da superfície da amostra [23]. Nesse trabalho, foi explorado a estabilidade da ponte líquida variando a umidade relativa, a distância $2 h$ da ponta do MFA até a base da amostra e os tamanhos das pontas do MFA. Posteriormente, outros estudos foram realizados com esse modelo, no qual o objetivo principal foi medir a força que a ponte líquida exerce na ponta do MFA. Foram realizados estudos em duas dimensões [24], três dimensões [25, 26] e com rugosidades diferentes das pontas do MFA [27].

Pode-se também encontrar estudos aplicados ao MFA, nos quais foram 
empregados a teoria do funcional da densidade [26, 28-30], a teoria cinética do funcional da densidade [31] e dinâmica molecular, utilizando os modelos TIP3P [32] e SPC [33] para a molécula de água. Esses dois últimos modelos consideram a molécula de água rígida e possuem três sítios de interação.

Os parâmetros do modelo TIP3P e do modelo SPC podem ser encontrados na Tabela 1.1, em que $\sigma_{L J}$ é a distância entre duas moléculas de água na qual o potencial de Lennard-Jones é nulo, $\varepsilon_{L J}$ é a profundidade do poço do potencial de Lennard-Jones da interação entre duas moléculas de água, $\mathrm{d}_{\mathrm{O}-\mathrm{H}}$ é o tamanho da ligação $(\mathrm{O}-\mathrm{H})$ entre o átomo de oxigênio $(\mathrm{O}), q_{H}$ é a carga em H, qo é a carga em O e H-Ô-H é o ângulo formado entre as duas ligações O-H. .

Tabela 1.1: Parâmetros dos modelos SPC e TIP3P da molécula de água.

\begin{tabular}{|l|c|c|}
\hline & TIP3P & SPC \\
\hline$\sigma$ & $3,15061 \AA$ & $3,166 \AA$ \\
\hline$\varepsilon$ & $0,6364 \mathrm{kJmol}^{-1}$ & $0,650 \mathrm{kJmol}^{-1}$ \\
\hline $\mathrm{d}_{\mathrm{O}-\mathrm{H}}$ & $0,9572 \AA$ & $1,0000 \AA$ \\
\hline $\mathrm{qH}_{\mathrm{H}}$ & $+0,4170 e$ & $+0,410 e$ \\
\hline $\mathrm{q}_{\mathrm{O}}$ & $-0,8340 e$ & $-0,8200 e$ \\
\hline $\mathrm{H}-\hat{\mathrm{O}}-\mathrm{H}$ & $104.52^{\circ}$ & $109,47^{\circ}$ \\
\hline
\end{tabular}

A escala dos modelos estatísticos e dinâmicos, descritos anteriormente, para a ponte líquida formada no MFA foi baseada em parâmetros dimensionais e energéticos da molécula de água. Utilizar esses modelos na escala macroscópica exigiria um custo computacional muito grande. Além disso, seria complexo descrever todas as variáveis envolvidas. Assim, são necessários outros modelos de fluidos para lidar com problemas na escala macroscópica. 
Um modelo do tipo gás de rede para pontes líquidas na escala macroscópica foi introduzido por Alencar e colaboradores [34]. Esse modelo foi aplicado para entender um problema da fisiologia pulmonar: a emissão do ruído de crepitação a partir da ruptura da ponte líquida. Neste trabalho, fez-se a hipótese de que a energia sonora, que gera o ruído de crepitação, provém da energia armazenada na superfície da ponte líquida antes da ruptura, para diferentes ângulos de contato. Os resultados numéricos foram comparados com cálculos analíticos utilizando integração numérica das equações variacionais que descrevem o equilíbrio da ponte líquida, validando, assim, o modelo de gás de rede para pontes líquidas macroscópicas .

Nesta dissertação, foi dado continuidade ao estudo desse modelo da ponte líquida desenvolvido por Alencar e colaboradores. Fez-se um estudo mecânico da ponte líquida para uma aplicação à fisiologia pulmonar, análogo ao realizado por Alencar e colaboradores [34], para estudar a emissão do ruído de crepitação durante a formação da ponte líquida. Além disso, fez-se um estudo termodinâmico desse modelo.

No estudo mecânico da formação da ponte líquida, mediu-se propriedades elásticas da ponte líquida alterando a distância entre as placas. Quando essa distância é aumentada, existe uma distância crítica $2 h_{c}$ em que a ponte líquida torna-se instável e se rompe. Após a ruptura, uma única gota será formada na superfície de uma das placas ou duas gotas, uma em cada placa. Quando as placas se aproximam, há uma distância crítica $2 h_{\text {min,c }}$ em que haverá a formação da ponte líquida. Nessa dissertação, verificou-se a existência de uma curva do tipo histerese na energia livre quando a altura das placas é alterada. Observou-se um efeito de tamanho finito da rede na for- 
mação das pontes líquidas quando as placas eram aproximadas. Foi realizado também o cálculo da força exercida nas placas pela ponte líquida.

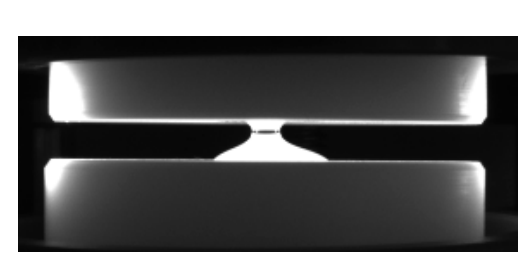

(a) Ponte líquida

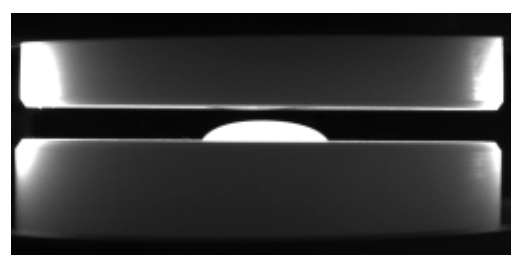

(b) Gota de água

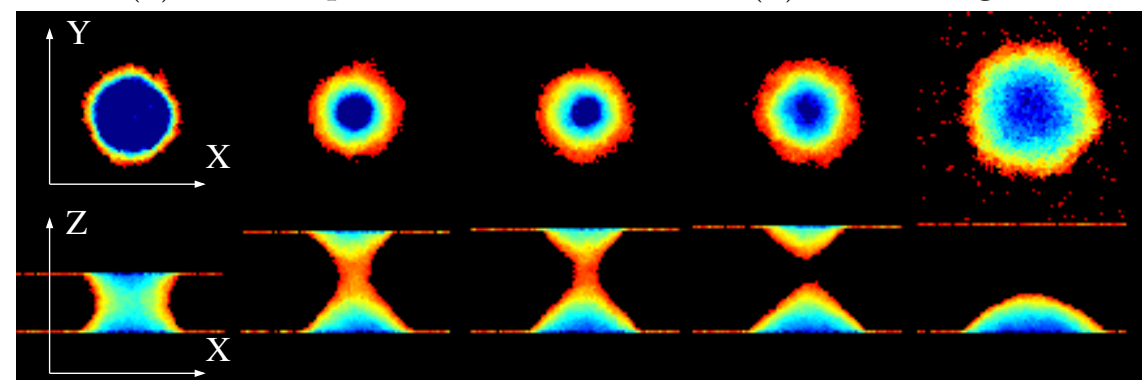

(c) Modelo da ponte líquida

Figura 1.3: (a) Ilustração de uma ponte líquida formada entre duas superfícies planas e (b) uma gota presa em uma das placas durante um experimento em que a ponte líquida é esticada até se romper. Nesse experimento, foi utilizado $200 \mu \ell$ de água e a placa plana era constituída de uma superfície hidrofílica. Pode-se observar que a ponte líquida e a gota estão assimétricas com relação ao eixo $Z$, pois o experimento foi realizado na presença da gravidade. As imagens foram feitas com um instrumento do nosso grupo utilizado para medir a curvatura das pontes líquidas. Em (c) tem-se uma ilustração de uma simulação do modelo gás de rede para pontes líquidas macroscópicas. O gradiente de cor do azul para o vermelho indica densidade de partículas do fluido: azul representa muitas partículas e vermelho poucas. A imagem superior é a projeção da ponte líquida no plano $X Y$ e a imagem inferior é a projeção da ponte líquida no plano $Z X$. A ponte líquida é esticada até se romper na altura critica $2 h_{c}$ e formar uma única gota. Neste caso, não existe a presença da gravidade.

Na Figura 1.3, pode-se observar imagens de uma ponte líquida e de uma gota obtidas a partir de um experimento e do modelo computacional proposto: na Fig. 1.3a tem-se a ponte líquida no momento da ruptura quando 
a distância entre placas está aumentando, na Fig. 1.3b tem-se apenas uma gota de água e na Fig. 1.3c a ilustração do modelo computacional quando se realiza uma simulação em que a distância entre as placas é aumentada.

Com relação ao estudo termodinâmico do modelo de gás de rede para pontes líquidas macroscópicas, fez-se um estudo da energia interna e do calor específico desse modelo. Inicialmente, estudou-se um sistema pequeno com apenas duas partículas de líquido para validar a metodologia. Posteriormente, o mesmo estudo foi realizado aumentando o tamanho da rede, para uma dada concentração de partículas de líquido no sistema. Então, fez-se um estudo da transição de fase com o aumento da temperatura e da densidade nesse modelo. Além disso, fez-se uma hipótese sobre o vínculo físico entre o modelo e os fluídos reais. Também utilizou-se o método "Overlapping Distribution" para calcular a energia livre do sistema e avaliar o efeito da entropia sobre a força exercida pela ponte líquida nas placas planas, uma informação importante em todos os aspectos físicos já discutidos.

No Capitulo 2, serão apresentados os modelos matemático e computacional para macroescala da ponte líquida. Serão discutidos também os métodos de Monte Carlo com algoritmo de Metropolis e o método "Overlapping Distribution" para o cálculo da energia livre. No Capitulo 3, serão apresentados os resultados obtidos da ponte líquida para quando a distância entre as placas é alterada e modelo do fluido para a escala macroscópica. Finalmente, no Capitulo 4 será feita a conclusão dos resultados aqui apresentados. 


\section{Capítulo 2}

\section{Modelos Analíticos e}

\section{Numéricos da Ponte Líquida}

Antes de entrar em detalhes sobre cálculo variacional utilizado para obter as propriedades físicas das pontes líquidas, será discutida a equação de YoungLaplace que é importante para o estudo da capilaridade. Posteriormente, serão realizados os cálculos para obter a curva da superfície de uma ponte líquida formada entre duas placas planas paralelas.

\subsection{A Equação de Young-Laplace}

A equação de Young-Laplace $[35,36]$ relaciona a diferença de pressão $\Delta P$ através da interface entre dois fluidos que possui uma tensão superficial $\gamma$ e uma curvatura $2 C$. Essa equação pode ser deduzida a partir de um exemplo simples que será discutido a seguir.

Seja um fluido 1 imerso em um fluido 2. Para minimizar a energia da 


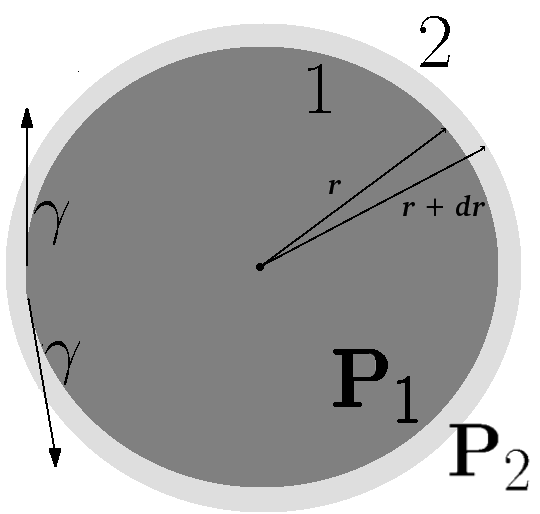

Figura 2.1: Ilustração do fluido $1 / 2$.

interface, o fluido 1 adquire o formato de uma esfera de raio $r$. Se a interface entre os fluidos 1 e 2 sofre uma variação infinitesimal do raio, $r^{\prime}=r+d r$, o trabalho infinitesimal $d W$ realizado pela pressão dos dois meios e pela tensão superficial na interface dos meios 1 e 2 é dado por:

$$
d W=-\mathbf{P}_{1} d V_{1}-\mathbf{P}_{2} d V_{2}+\gamma d A,
$$

em que $d V_{1}$ e $d V_{2}$ são, respectivamente, as variações infinitesimais do volume do fluido 1 e do fluido $2 ; \mathbf{P}_{1}$ e $\mathbf{P}_{2}$ são, respectivamente, as pressões do fluido 1 e do fluido $2 ; \gamma$ é a tensão superficial da interface dos fluidos 1 e $2 ; d A$ á uma variação infinitesimal da área interfacial dos dois meios.

No equilíbrio, tem-se que $d W=0$. Sendo $d V_{1}=4 \pi r^{2} d r, d A=8 \pi r d r$ e $d V_{2}=-d V_{1}$, pode-se obter:

$$
0=-\mathbf{P}_{1} 4 \pi r^{2} d r-\mathbf{P}_{2}\left(-4 \pi r^{2} d r\right)+\gamma 8 \pi r d r
$$




$$
\Delta P=\mathbf{P}_{1}-\mathbf{P}_{2}=\frac{2}{r} \gamma
$$

que é a equação de Young-Laplace para uma interface esférica de raio $r$. Na Equação 2.2, 2/r é a curvatura de uma esfera de raio $r$. Assim, a forma geral da equação de Young-Laplace para qualquer interface entre dois fluidos pode ser escrita como:

$$
\Delta P=\gamma 2 C,
$$

onde $2 C$ é a curvatura da interface,

$$
2 C=\left(\frac{1}{R_{1}}+\frac{1}{R_{2}}\right)
$$

e $R_{1}$ e $R_{2}$ são os raios principais da curvatura, ver Fig. 2.2.

Para calcular os raios principais de curvatura em um ponto $M$ de uma superfície qualquer, é necessário traçar uma reta normal $\xi$ através desse ponto. Então, dois planos ortogonais entre si devem ser desenhados de modo a conter a reta $\xi$. A interseção de cada um dos planos com a superfície terá um raio de curvatura contido em cada um dos planos. Na Figura 2.2, pode-se observar os raios de curvatura $R_{1}$ e $R_{2}$ contidos em cada um dos planos perpendiculares, sendo o plano que contem $R_{2}$ está evidenciado pela cor cinza. O raio de curvatura que estiver contido dentro da superfície geométrica de interesse será positivo e o raio de curvatura que estiver fora dessa geometria será negativo. Para o cálculo da curvatura da ponte líquida serão utilizadas coordenadas cilíndricas. Assim, $2 C$ pode ser reescrito em coordenadas cilíndricas como: 


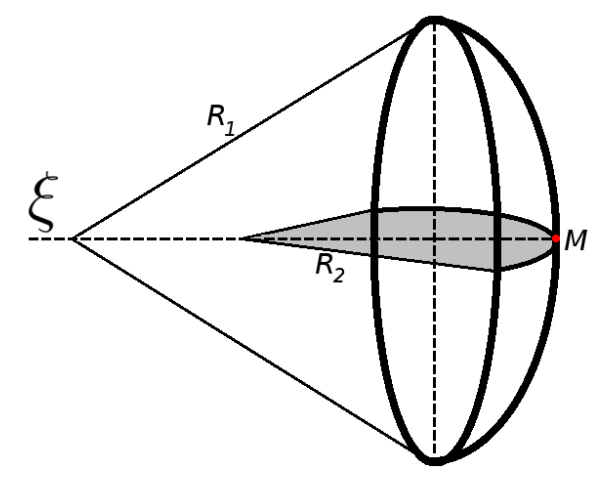

Figura 2.2: Ilustração dos raios principais de curvatura $R_{1}$ e $R_{2}$ que estão contidos em dois planos perpendiculares entre si, cuja interseção forma a reta normal $\xi$. O ponto $M$ está contido na superfície do fluido pelo qual passa a reta normal $\xi$.

$$
2 C=-\frac{\rho^{\prime \prime}(z)}{{\sqrt{1+\rho^{\prime}(z)^{2}}}^{3}}+\frac{1}{\rho(z) \sqrt{1+z^{\prime}(\rho)^{2}}} .
$$

em que o primeiro termo de $2 C$ é a curvatura contida no plano que passa através do eixo de simetria e o segundo termo é a curvatura contida num plano através da direção azimutal [37].

\subsection{Soluções Analíticas}

O estudo da estabilidade de pontes líquidas e outros fenômenos envolvendo a ação da tensão superficial na superfície de fluidos têm sido amplamente estudados $[38,39]$. Pode-se encontrar modelagens matemáticas sobre a estabilidade, curvatura e força de uma ponte líquida formada entre duas placas planas [40-43], em partículas esféricas iguais [44] e de tamanhos diferentes, sejam lisas [45] ou rugosas [46]. Pode-se encontrar também estudos para o processo de sinterização de fase líquida [3], na fisiologia pulmonar [47, 48] e 


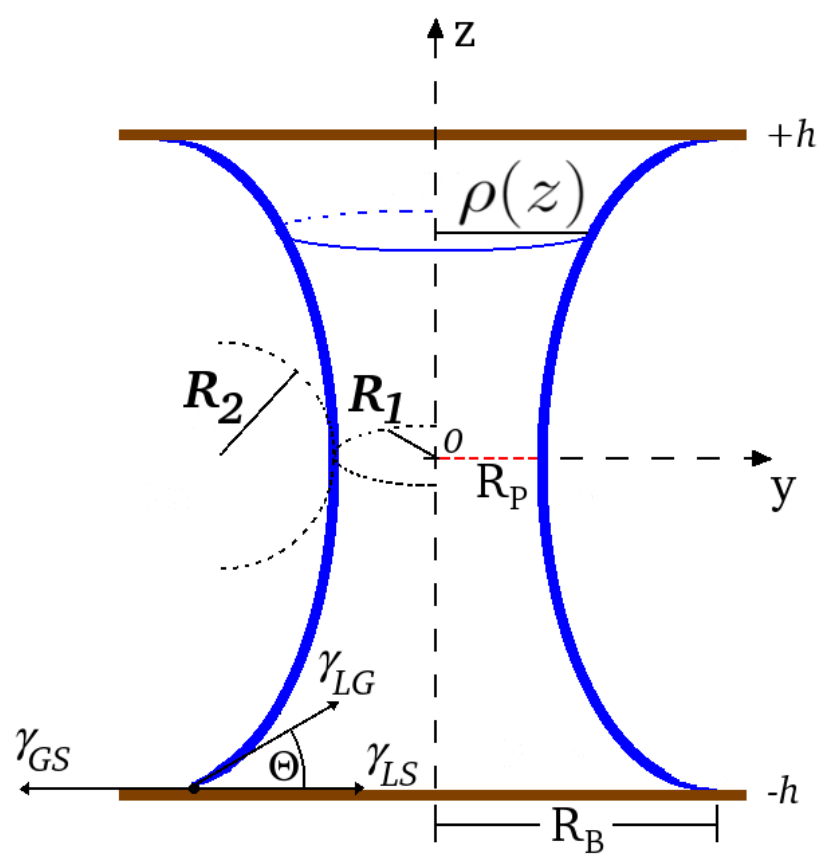

Figura 2.3: Ilustração de uma ponte líquida formada entre duas placas planas nas alturas $+h \mathrm{e}-h$. A superfície é dada pela rotação do raio $\rho(z)$ em torno do eixo $Z$. A curvatura da superfície $C(\Omega, h, \Theta)$ depende do volume da ponte líquida $\Omega$, da meia altura $h$ da ponte líquida e do ângulo de contato $\Theta$. O ângulo de contato $\Theta$ pode ser obtido das tensões superficiais $\gamma_{g s}, \gamma_{g l}$ e $\gamma_{l s}$. O raio destacado em vermelho $R_{P}$ é o raio do "pescoço" da ponte líquida. $R_{B}$ é o raio da base da ponte líquida.

sobre uma ponte líquida formada entre um plano e uma esfera [49].

Seja uma ponte líquida formada axialmente entre duas placas planas e paralelas dispostas nas alturas $-h$ e $+h$, e a origem do sistema está no centro da ponte líquida, como pode ser verificado na Fig. 2.3. O sistema de coordenadas adotado é o cilíndrico e o raio da ponte líquida é dado por $\rho(z)$. A superfície da ponte líquida será formada pela rotação do raio $\rho(z)$, sendo $-h<z<h$, em torno o eixo $Z$ da ponte líquida. Uma vez estabelecido o equilíbrio, a ponte líquida terá uma superfície com uma curvatura $C(\Omega, h, \Theta)$, que dependerá somente dos parâmetros $\Omega, h$ e $\Theta$ que representam, respecti- 
vamente, o volume de líquido, a meia altura e o ângulo de contato da ponte líquida.

Todas as características relevantes da ponte líquida entre os dois planos foram determinados em termos de integrais elípticas que dependem do ângulo de contato $\Theta$ e de um parâmetro adimensional $a$ que é a razão entre o raio do "pescoço" da ponte líquida $R_{P}$ e do raio da base $R_{B}: a=R_{P} / R_{B}$, como pode ser observado na Fig. 2.3. O "pescoço" da ponte líquida é definido como a região da ponte que possui o menor raio, e o raio base como o raio da região em que a ponte toca os planos.

A energia total $E_{\text {tot }}$ da ponte líquida pode ser escrita na forma geral como:

$$
E_{\text {tot }}=E_{\text {interf }}+E_{\text {cin }}+E_{\text {pot }}+U
$$

em que $E_{\text {interf }}$ é a energia armazenada na interface entre dois meios, $E_{\text {cin }}$ é a energia cinética da ponte líquida, $E_{\text {pot }}$ é a energia potencial gravitacional da ponte líquida e $U$ é a energia interna da ponte líquida. Como o sistema está em equilíbrio e na ausência da gravidade, o termo $E_{\text {cin }}$ é nulo e o termo $E_{\text {pot }}$ é constante. O termo $U$ também é constante pois não existe alteração na quantidade de matéria.

Por sua vez, a energia interfacial pode ser escrita como:

$$
E_{\text {interf }}=\gamma_{g l} A_{R}+\gamma_{l s} A_{B}+\gamma_{g s}\left(A_{T}-A_{B}\right)-\gamma_{g s} A_{T}
$$

em que $\gamma_{g l}$ e $A_{R}$ são, respectivamente, a tensão superficial e a área da interface gás-líquido; $\gamma_{l s}$ e $A_{B}$ são, respectivamente, a tensão superficial e a área da interface líquido-sólido; $\gamma_{g s}$ e $A_{T}$ são, respectivamente, a área total e a tensão 
superficial da interface gás-sólido. Como será realizada a minimização da energia, os termos constantes podem ser retirados. Assim, para efeito da minimização da energia pode-se considerar apenas $E_{\text {interf }}$, logo:

$$
E_{\text {interf }}=\gamma_{g l} A_{R}+\left(\gamma_{l s}-\gamma_{g s}\right) A_{B}
$$

A área da base $A_{B}$ pode ser reescrita em termos do raio da ponte líquida $\rho(z) \operatorname{nos}$ pontos $z=-h$ e $z=h$ :

$$
A_{B}=\pi\left(\rho(h)^{2}+\rho(-h)^{2}\right) .
$$

A área da interface ar-líquido $A_{R}$ pode ser reescrita como:

$$
A_{R}=\int_{h}^{-h} 2 \pi \rho(z) \sqrt{1+\rho^{\prime}(z)^{2}} d z
$$

em que $2 \pi \rho(z)$ é o comprimento de uma seção transversal da ponte líquida na altura $z$ e $\sqrt{1+\rho^{\prime}(z)^{2}} d z$ é um elemento infinitesimal do arco da função $\rho(z)$.

Assim, pode-se reescrever a energia total da ponte líquida como:

$$
E_{\text {interf }}=\pi\left(\gamma_{s l}-\gamma_{g s}\right)\left(\rho(h)^{2}+\rho(-h)^{2}\right)+\gamma_{g l} \int_{h}^{-h} 2 \pi \rho(z) \sqrt{1+\rho^{\prime}(z)^{2}} d z
$$

As possíveis superfícies geradas por pontes líquidas formam uma classe de estruturas geométricas denominadas superfícies de Delaunay. Se $\rho(z)$ é uma elipse, então $C>0$, e a superfície é conhecida como uma subclasse de Delaunay denominada ondulóide. Se $\rho(z)$ é uma hipérbole, então $C<0$, e a 
superfície é conhecida como nodóide. Se $\rho(z)$ é uma parábola, então $C=0$, e a superfície é conhecida como catenóide. As formas que essas superfícies assumem dependem das tensões superficiais entre os meios $\left(\gamma_{g l}, \gamma_{g s}\right.$ e $\left.\gamma_{s l}\right)$, que por sua vez estão relacionadas com o ângulo de contato $\Theta$, dado pela Eq. 2.16.

Para que a ponte líquida esteja em equilíbrio, a curvatura média $C(\Omega, h, \Theta)$ deve ser constante no tempo. Nesse caso, a diferença de pressão $\Delta P$ na Eq. 2.3 também se mantém constante, caracterizando um sistema em equilíbrio.

Outras condições de equilíbrio devem ser estabelecidas: não pode haver nenhum ponto de inflexão na superfície, ou seja, $\rho^{\prime \prime}(z) \neq 0$; o perfil da ponte líquida deve pertencer a uma família de soluções contínuas para as quais $\frac{\partial \Omega}{\partial C}<0$ para qualquer $\Omega \geq \Omega_{0}$, ou seja, quando a curvatura $2 C$ aumentar o volume $\Omega$ diminui.

Para calcular a função $\rho(z)$ que gera o perfil da superfície da ponte líquida, deve-se fazer o cálculo da minimização da energia total do sistema $E_{\text {interf }}$ com o vínculo de que o $\Omega$ do sistema seja constante. O cálculo analítico dessas superfícies esta discutido e pode ser encontrado em detalhes em outras fontes [34, 37, 39]. Sendo assim, a seguir serão apresentados apenas as equações necessárias para se obter: a altura mínima para a formação de uma ponte líquida a partir de uma gota $2 h_{\mathrm{min}, 1}$ ou duas gotas $2 h_{\mathrm{min}, 2}$ presas nas placas planas; a energia livre de uma única gota presa na placa $\mathcal{F}_{0,1}$; a energia livre da ponte líquida presa na placa $\mathcal{F}_{b}$, que são objetos de estudo dessa dissertação.

Para o caso da formação de duas gotas, uma em cada um dos planos, só é 


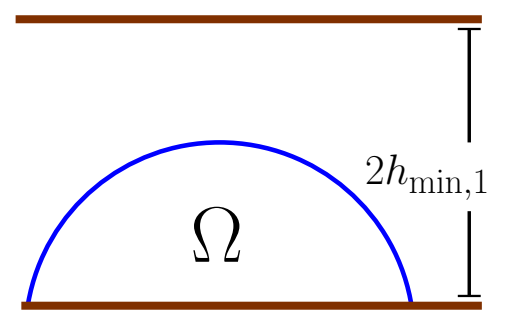

(a) Uma gota

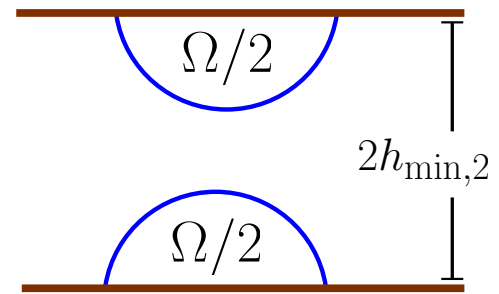

(b) Duas gota

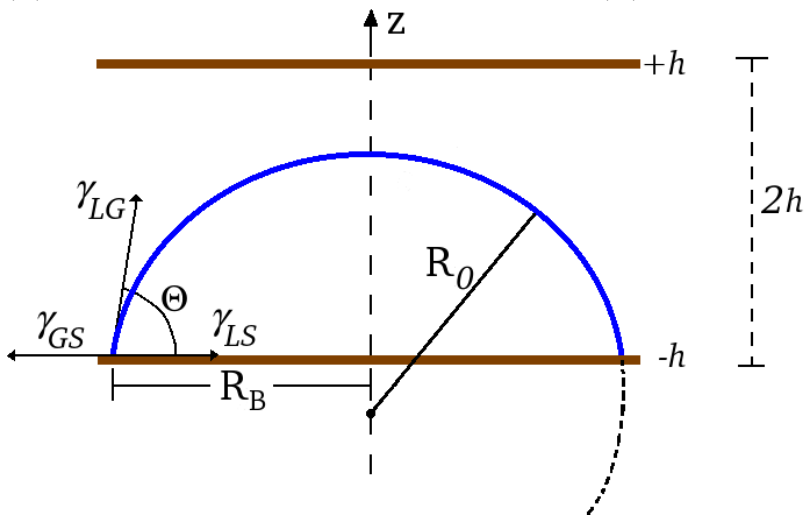

(c) Geometria da gota

Figura 2.4: Ilustração de uma (a) e duas (b) gotas presas entre as placas. Pode-se observar em (b) que que as gotas possuem metade do volume $(\Omega / 2)$ de uma única gota. A altura crítica para a formação da ponte líquida a partir de uma gota é $2 h_{\min , 1}$ e a partir de duas gotas é $2 h_{\min , 2}$. Em (c) estão representados os parâmetros geométricos para descrever a gota: ângulo de contato $\Theta$, tensões superficiais $\left(\gamma_{g s}, \gamma_{l g}\right.$ e $\left.\gamma_{l s}\right)$, raio $R_{B}$ da intersecção de um plano com uma esfera de raio $R_{0}$.

possível no modelo matemático e se as duas gotas forem simétricas. No caso do modelo matemático, essas gotas terão a mesma curvatura $2 C$. Assim, de acordo com a Eq. 2.3 a diferença de pressão $\Delta P$ será a mesma para as duas gotas, e por isso permanecerão em equilíbrio. No caso do modelo gás de rede da ponte líquida, como também no caso real, exite uma flutuação térmica do sistema. Esse efeito térmico pode fazer com que a curvatura de uma das gotas se torne maior. Consequentemente, essa gota vai coalescer na gota com $2 C$ menor, como pode ser verificado na Fig. 1.3c. 
Se existir apenas uma gota de líquido presa a um dos planos, o formato adquirido pela gota será de um seguimento esférico de raio $R_{0}$, com o centro na posição $\left(x=0, y=0, z= \pm\left[h+R_{0} \cos \Theta\right]\right)$ formando uma interseção circular com o plano de raio $R_{B}=R_{0} \sin \Theta$, onde:

$$
R_{0}=\left(\frac{6 \Omega}{2 \pi\left(2-3 \cos \Theta+\cos ^{3} \Theta\right)}\right)^{1 / 3},
$$

como pode ser verificado na Fig. 2.4a e na Fig. 2.4c. Nesse caso, a menor separação dos planos antes da formação da ponte líquida é dada por:

$$
2 h_{\min , 1}=R_{0}(1-\cos \Theta) .
$$

A Figura 2.4b ilustra as duas gotas de acordo com o modelo matemático. A distância mínima de separação entre as placas para a formação da ponte líquida a partir de duas gotas simétricas presas entre as placas é dada pela soma das alturas das duas gotas:

$$
2 h_{\min , 2}=R_{0}^{\prime}(1-\cos \Theta)+R_{0}^{\prime}(1-\cos \Theta) \text {. }
$$

em que $R_{0}{ }^{\prime}$ é o raio da gota aproximada por uma esfera com metade do volume do caso com somente uma gota. Assim:

$$
R_{0}{ }^{\prime}=\left(\frac{6 \frac{\Omega}{2}}{2 \pi\left(2-3 \cos \Theta+\cos ^{3} \Theta\right)}\right)^{1 / 3}
$$

Desse modo, a Eq. 2.8 pode ser reescrita como:

$$
2 h_{\min , 2}=2^{2 / 3} R_{0}(1-\cos \Theta)
$$




$$
2 h_{\min , 2}=2^{2 / 3} h_{\min , 1}
$$

A energia livre da ponte líquida $\mathcal{F}_{b}\left(\Omega, h_{\min }\right)$ é dada por [34]:

$$
\mathcal{F}_{b}(a) \equiv 2 \pi h_{\min }^{2} \frac{2 \sigma(a, 1)-\cos \Theta}{u(a, 1)^{2}}
$$

e a energia livre de uma única gota $\mathcal{F}_{0,1}(\Omega)$ é dada por:

$$
\mathcal{F}_{0,1}=\pi \gamma R_{0}^{2}\left[2(1-\cos \Theta)-\sin ^{2} \Theta \cos \Theta\right]
$$

sendo que sempre $\mathcal{F}_{b}\left(\Omega, h_{\text {min }}\right)<\mathcal{F}_{0,1}$ onde $a$ é determinado pela equação

$$
\Omega=2 \pi h_{\min }^{3} \frac{v(a, 1)}{u(a, 1)^{3}},
$$

e $u(a, 1), v(a, 1)$, e $\sigma(a, 1)$ podem ser expressos em termos das integrais elípticas de Legendre $F(k, \phi)$ e $E(k, \phi)$ como segue abaixo:

$$
\begin{aligned}
u(a, r)= & a \tilde{F}\left(k, \phi_{r}\right)+b \tilde{E}\left(k, \phi_{r}\right), \\
\sigma(a, r)= & (b+a) b \tilde{E}\left(k, \phi_{r}\right), \\
v(a, r)= & {\left[-\mathcal{R}(a, r)-a^{2} b \tilde{F}\left(k, \phi_{r}\right)\right.} \\
& \left.+b\left[3 a b+2\left(a^{2}+b^{2}\right)\right] \tilde{E}\left(k, \phi_{r}\right)\right] / 3,
\end{aligned}
$$

em que $b, k, \mathcal{R}(a, r), \phi_{r}, \tilde{F}(k, \phi)$ e $\tilde{E}(k, \phi)$ são definidos abaixo : 


$$
\begin{array}{ll}
b= & \left(\frac{1-a \sin \Theta}{\sin \Theta-a}\right), \\
k= & s \sqrt{b^{2}-a^{2}} / b, \\
\mathcal{R}(a, r)= & r \sqrt{\left(r^{2}-a^{2}\right)\left(b^{2}-r^{2}\right)}, \\
\phi_{r}= & \arcsin \sqrt{\left(b^{2}-r^{2}\right) /\left(b^{2}-a^{2}\right)}, \\
\tilde{F}(k, \phi)= & F(k, \pi / 2)-F(k, \phi), \\
\tilde{E}(k, \phi)= & E(k, \pi / 2)-E(k, \phi) .
\end{array}
$$

Essas equações serão utilizadas na análise para validar os resultados do modelo. A seguir, será descrito o modelo de gás de rede para a modelagem de pontes líquidas macroscópicas.

\subsection{Modelo de Gás de Rede}

O modelo da ponte líquida utilizado nessa dissertação está sendo desenvolvido para lidar com problemas na escala macroscópica. Os sistemas em estudo, características do líquido, gás e parede plana, serão caracterizados pelo ângulo de contato $\Theta$ que é dado por:

$$
\cos \Theta=\frac{\gamma_{l s}-\gamma_{s g}}{\gamma_{g l}}
$$

Este é um modelo tipo gás de rede em que as partículas do sistema interagem com duas paredes planas e paralelas separadas pela distância $2 h$. Podem existir três tipos de partículas na rede: líquido $(l)$, gás $(g)$ e sólido $(s)$. Cada uma dessas partículas do modelo representa uma unidade macroscópica. Assim, uma partícula de líquido pode ser entendida como uma pequena gotícula e uma partícula de gás como uma pequena caixa contendo 
gás difundido. As partículas de sólido formam as paredes paralelas, na qual a ponte líquida está fixada, e podem se mover na direção $z$ para alterar a altura da ponte líquida. Cada partícula da rede possui 26 sítios de interação: $6+12+8$, com distância $1, \sqrt{2}$ e $\sqrt{3}$, respectivamente. O valor da interação para as distâncias $\sqrt{2}$ e $\sqrt{3}$ foram reduzidas para simular um potencial de longo alcance; a interação entre as partículas de sólido e líquido com gás foram restritas para a distância 1 [34].

As interações nas distâncias $\sqrt{2}$ e $\sqrt{3}$ são necessárias para que a relação área/volume seja minimizada, como acontece com um fluido em equilíbrio. Para ilustrar esse fato, a Fig. 2.5 está mostrando dois exemplos de simulações de uma gota. Para que essa simulação fosse possível, as paredes planas foram removidas constituindo, assim, um sistema com partículas de líquido e gás. Foram utilizados 4000 partículas de líquido e uma rede de $60 \times 60 \times 60$. Para formar as gotas, inicialmente o sistema estava a uma temperatura elevada, $T=20 \mathrm{~J} / k_{\mathrm{B}}$, em que as partículas de líquido estavam vaporizadas. Então, o sistema foi esfriado até $T=0 J / k_{\mathrm{B}}$, formando uma gota. A Figura. $2.5 \mathrm{~b}$ está mostrando a gota quando se considera somente interações na distância 1. A superfície que minimiza a energia dessa gota é um cubo. A Figura 2.5c esta mostrando a gota quando se considera interações nas distâncias $1, \sqrt{2}$ e $\sqrt{3}$. Pode-se observar a formação de uma superfície esférica, que é a forma da superfície observada quando a energia é minimizada.

A energia desse modelo para pontes líquidas macroscópicas é dada pelo hamiltoniano:

$$
\mathcal{H}=\frac{1}{2} \sum_{1}^{V} E_{m}
$$




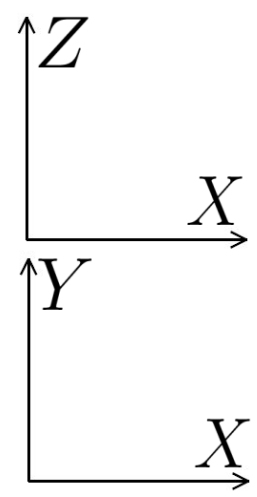

(a)

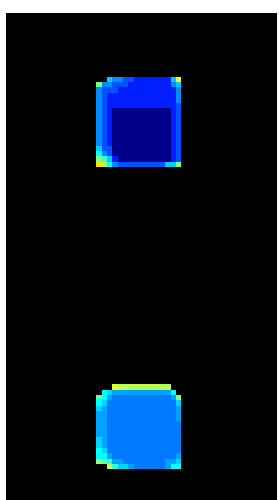

(b)

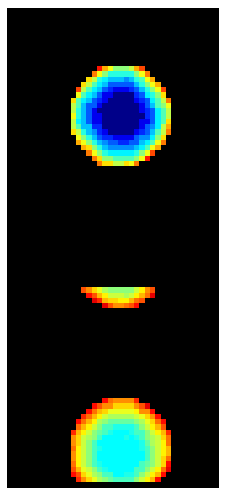

(c)

Figura 2.5: Comparação de duas simulações de gota na temperatura de $T=$ $0 \mathrm{~J} / k_{\mathrm{B}}$. Foram utilizados 4000 partículas de líquido e uma rede de $60 \times 60 \times 60$. Em (a) tem-se a ilustração do sistema de coordenadas da rede. Em (b) podese observar o que acontece quando se considera somente interação na distância 1, e em (c) interações de nas distâncias $1, \sqrt{2}$ e $\sqrt{3}$.

sendo,

$$
E_{m}=\sum_{k=1}^{26} J_{i(m) j(k)},
$$

em que $E_{m}$ é o hamiltoniano do sítio $m$ da rede, $J_{i j}$ é a energia potencial de interação de cada uma das combinações possíveis das partículas $i, j=s, l, g$ e $k$ são os sítios vizinhos ao sítio $m$. Os parâmetros $J_{i j}$ são ajustados de modo a simular um gás de rede e também obter um ângulo de contato $\Theta$ que é o parâmetro que caracteriza o sistema em estudo. Analogamente ao gás de rede, considerou-se a interação entre sítios ocupados como sendo $J_{l l}=-1$. Nós consideramos que os sítios vazios representam gás e a interação entre esses sítios é dada por $J_{g g}=0$. Considerou-se, também, que existe uma interação fraca entre as partículas de gás com sólido $\left(J_{g s}\right)$ e com líquido $\left(J_{g l}\right)$, fato desconsiderado no modelo clássico do gás de rede. Os valores dessas interações estão nas Tabelas 2.1, 2.2 e 2.3. Na Seção 3.2 serão avaliados os 
efeitos das interações das distâncias $\sqrt{2}$ e $\sqrt{3}$. Para simplificar a notação, serão utilizados os índices $J_{i j}^{1}, J_{i j}^{2}$ e $J_{i j}^{3}$, respectivamente, para as interações nas distâncias $1, \sqrt{2}$ e $\sqrt{3}$.

Tabela 2.1: Parâmetros das interações $J_{i j}^{1}$ entre as partículas na distância 1.

\begin{tabular}{|c|c|c|c|}
\hline & gás & líquido & sólido \\
\hline gás & $J_{g g}=0$ & $J_{g l}=-0,02$ & $J_{g s}=-0,032$ \\
\hline líquido & $J_{g l}=J_{l g}$ & $J_{l l}=-1,00$ & $J_{l s}=-1,45$ \\
\hline sólido & $J_{g s}=J_{s g}$ & $J_{l s}=J_{s l}$ & \\
\hline
\end{tabular}

Tabela 2.2: Parâmetros das interações $J_{i j}^{2}$ entre as partículas na distância $\sqrt{2}$.

\begin{tabular}{|c|c|c|c|}
\hline & gás & líquido & sólido \\
\hline gás & $J_{g g}=0$ & $J_{g l}=0$ & $J_{g s}=0$ \\
\hline líquido & $J_{g l}=J_{l g}$ & $J_{l l}=-0,7$ & $J_{l s}=-0,7$ \\
\hline sólido & $J_{g s}=J_{s g}$ & $J_{l s}=J_{s l}$ & \\
\hline
\end{tabular}

Tabela 2.3: Parâmetros das interações $J_{i j}^{3}$ entre as partículas na distância $\sqrt{3}$.

\begin{tabular}{|c|c|c|c|}
\hline & gás & líquido & sólido \\
\hline gás & $J_{g g}=0$ & $J_{g l}=0$ & $J_{g s}=0$ \\
\hline líquido & $J_{g l}=J_{l g}$ & $J_{l l}=-0,4$ & $J_{l s}=0$ \\
\hline sólido & $J_{g s}=J_{s g}$ & $J_{l s}=J_{s l}$ & \\
\hline
\end{tabular}

Alterando as interações $J_{i j}$ é possível alterar as tensões superficiais e assim o valor do ângulo de contato $\Theta$ [34]. Nas análises realizadas nessa dissertação, o valor de $\Theta$ foi ajustado alterando o valor da interação $J_{s l}$. Por exemplo, para $J_{s l}=-1,45$ obtém-se $\Theta=50^{\circ}$. Desse modo, é possível caracterizar o sistema em estudo. 
As simulações desse modelo foram realizadas com método de Monte Carlo com algoritmo de Metropolis [50,51]. Não há nenhuma troca entre partículas iguais, e as trocas entre partículas de gás e líquido são realizadas aleatoriamente de acordo com a dinâmica de Kawasaki [52] e ponderadas pelo fator de Boltzmann, enquanto que as partículas de sólido são mantidas fixas para manter a geometria das placas. A seguir, será descrito o método de Monte Carlo com algoritmo de Metropolis e em seguida a dinâmica de Kawasaki.

\subsection{Método Monte Carlo com Algoritmo de Metropolis}

Simulação computacional é uma poderosa ferramenta para o entendimento de problemas físicos complexos para os quais não existe uma solução analítica [53, 54]. O método de Monte Carlo com algoritmo de Metropolis é uma ferramenta utilizada para obter configurações aleatórias do espaço de fase de um sistema com uma hamiltoniana conhecida, onde cada configuração obtida com esse método depende somente da configuração anterior, definindo uma cadeia de Markov [52].

Dado um sistema com $\mathcal{N}$ possíveis estados acessíveis $\sigma_{j}$, o estado do sistema no instante de tempo $t$ é dado por $\Sigma_{t}=\sigma_{j}$. A probabilidade de obter um estado $\sigma_{j}$ dado a ocorrência de um estado $\sigma_{k}$, é dado pela probabilidade condicional $p\left(\sigma_{j} \mid \sigma_{k}\right)$. Em uma cadeia de Markov, a ocorrência do estado $\Sigma_{t}$ depende somente do estado anterior do sistema $\Sigma_{t-1}$, de modo que a de-

pendência dos estados anteriores a $t-1$ é nula: $p\left(\Sigma_{t-2} ; \Sigma_{t-3} ; \ldots ; \Sigma_{t-\infty} \mid \Sigma_{t}\right)=$ 
0. Assim, a probabilidade de obter o sistema no tempo $t$ com estado $\sigma_{j}$ $\left(\mathcal{P}\left(\Sigma_{t}=\sigma_{j}\right)\right)$ é dada por:

$$
\mathcal{P}\left(\Sigma_{t}=\sigma_{j}\right)=w_{j k} \mathcal{P}\left(\Sigma_{t-1}=\sigma_{k}\right)
$$

onde,

$$
w_{j k}=p\left(\Sigma_{t-1}=\sigma_{j} \mid \Sigma_{t}=\sigma_{k}\right)
$$

é a probabilidade de transição do estado $\sigma_{k}$ no instante $t-1$ para o estado $\sigma_{j}$ no instante $t$ que deve satisfazer as seguintes condições:

$$
w_{j k} \geqslant 0 \quad \quad \sum_{j} w_{j k}=1
$$

A probabilidade de transição entre os estados $\sigma_{j}$ considerando agora o tempo $t$ uma variável contínua pode ser obtida pela equação mestra,

$$
\frac{d \mathcal{P}\left(\sigma_{j}, t\right)}{d t}=-\sum_{k} w_{j k} \mathcal{P}\left(\sigma_{j}, t\right)+\sum_{k} w_{k j} \mathcal{P}\left(\sigma_{k}, t\right)
$$

onde $\mathcal{P}\left(\Sigma_{t}=\sigma_{j}\right)=\mathcal{P}\left(\sigma_{j}, t\right)$. No equilíbrio, a probabilidade de transição para um dado estado se torna independente do tempo. Assim:

$$
\frac{d \mathcal{P}\left(\sigma_{j}, t\right)}{d t}=0
$$

Logo, da Eq. 2.19 pode-se obter:

$$
w_{j k} \mathcal{P}\left(\sigma_{j}, t\right)=w_{k j} \mathcal{P}\left(\sigma_{k}, t\right)
$$

No ensemble canônico, a probabilidade de ocorrência do estado $\mathcal{P}\left(\sigma_{j}, t\right)$ 
é dado por:

$$
\mathcal{P}\left(\sigma_{j}, t\right)=\frac{e^{-\frac{E_{\sigma_{j}}}{k_{\mathrm{B}} T}}}{\mathcal{Z}},
$$

em que $\mathcal{Z}$ é a função de partição, $k_{\mathrm{B}}$ é a constante de Boltzmann, $T$ é a temperatura e $E_{\sigma_{j}}$ é a energia do estado $\sigma_{j}$. Aplicando a Eq. 2.21 na Eq. 2.20, pode-se obter:

$$
\begin{array}{ll}
w_{j k}=w_{k j} e^{-\frac{\Delta E}{k_{\mathrm{B}} T}} & \Delta E>0 \\
w_{j k}=w_{k j} & \Delta E<0,
\end{array}
$$

em que $\Delta E=E_{s_{j}}-E_{s_{k}}$. De acordo com a literatura, costuma-se escrever $w_{k j}=1 / \tau$, onde $\tau$ é uma unidade de tempo de Monte Carlo. Desse modo, $w_{k j}$ pode ser interpretado como uma probabilidade de transição por unidade de tempo.

A seguir será descrito o algoritmo para gerar as configurações do método de Monte Carlo com amostragem de Metropolis. Posteriormente a dinâmica de Kawasaki será apresentada.

1. Sorteia um estado inicial $\Sigma_{t}=\sigma_{j}$

2. Sorteia um estado final $\Sigma_{t+1}=\sigma_{k}$

3. Calcula a diferença de energia entre esses dois estados: $\Delta E=E_{\sigma_{j}}-E_{\sigma_{k}}$

(a) Se $\Delta E<0$ :

- A configuração $\Sigma_{t+1}=\sigma_{k}$ é aceita

- Uma nova configuração deve ser gerada no passo 2: $\Sigma_{t+2}$ 
(b) Se $\Delta E>0$ :

i. Faz-se o teste $w_{j k}=e^{-\frac{\Delta E}{k_{\mathrm{B}} T}}$

ii. Gerar um número aleatório $\alpha$, tal que $0<\alpha<1$

iii. Se $\alpha<w_{k j}$ :

- A configuração $\Sigma_{t+1}=\sigma_{k}$ é aceita

- Uma nova configuração deve ser gerada no passo 2: $\Sigma_{t+2}$

iv. Se $\alpha>w_{k j}$ :

- A configuração $\Sigma_{t+1}=\sigma_{k}$ é recusada

- Uma nova configuração deve ser gerada no passo 2: $\Sigma_{t+2}$

\subsection{Dinâmica de Kawasaki}

No modelo de Ising os possíveis estados de spins podem ser $\sigma_{i}= \pm 1$. Em uma dinâmica clássica do modelo de Ising com Método de Monte Carlo um sítio da rede é sorteado e então calcula-se a probabilidade desse spin alterar seu valor: $\sigma_{i}=+1$ para $\sigma_{i}=-1$, e vice-versa. Isso faz com que ocorram flutuações na energia e na magnetização.

Por sua vez, na dinâmica de Kawasaki [52] isso ocorre de forma diferente, ou seja, a proporção entre spins com valores $\sigma_{i}=+1$ e $\sigma_{i}=-1$ permanece constante, mantendo a magnetização constante. Na dinâmica de Kawasaki com Método de Monte Carlo dois spins da rede são sorteados. Em seguida calcula-se a probabilidade de troca desses spins. Se a nova configuração for aceita, os spins mudam de posição mantendo a mesma magnetização. A 
Figura 2.6 faz uma comparação da dinâmica clássica de uma simulação do modelo de Ising e da dinâmica de Kawasaki.

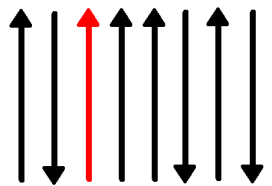

(a) Ising: I

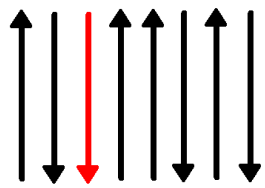

(b) Ising: $\mathrm{F}$

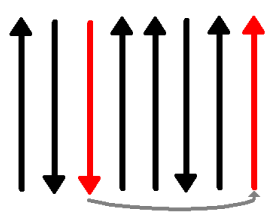

(c) Kawasaki: I

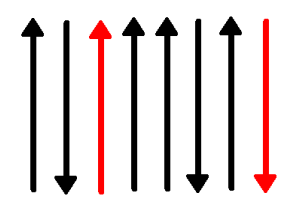

(d) Kawasaki: F

Figura 2.6: Ilustração comparando a dinâmica clássica de Ising com a dinâmica de Kawasaki. As setas pretas representam spins que não sofreram trocas. As setas vermelhas ilustram spins sorteados antes e depois das trocas (a) e (b) são as configurações inicial (I) e final (F) do Ising clássico, respectivamente; (c) e (d) são as configurações inicial (I) e final (F) da dinâmica de Kawasaki, respectivamente.

\subsection{Energia Livre: Método "Overlapping Dis- tribution"}

Para problemas simples, como uma série de osciladores harmônicos desacoplados e o sólido de Einstein, o cálculo da energia livre é trivial. Entretanto, problemas complexos, como o caso da ponte líquida estudada nesta dissertação, $\mathcal{F}(N, V, T)$ é uma grandeza difícil de ser calculada. Assim, existem diferentes métodos para calcular a energia livre, como integração termodinâmica, potencial de força média, método de "Umbrella" e o "Overlapping Distribution" que será discutido a seguir.

A energia livre $\mathcal{F}(N, V, T)$ de um sistema no ensemble canônico pode ser escrita da seguinte forma: 


$$
\mathcal{F}(N, V, T)=-k_{\mathrm{B}} T \ln (\mathcal{Z})
$$

com,

$$
\mathcal{Z}=h_{P}^{-3 N} \iint \exp \left\{\frac{-\mathcal{H}(\mathbf{p}, \mathbf{r})}{k_{\mathrm{B}} T}\right\} d \mathbf{p} d \mathbf{r}
$$

onde $\mathcal{Z}, N, V$ e $T$ representam, respectivamente, a função de partição, o número de partículas, volume e temperatura do sistema; $\mathcal{H}(\mathbf{p}, \mathbf{r})$ é o hamiltoniano do sistema, sendo $\mathbf{p}$ e $\mathbf{r}$ as coordenadas de momento e posição, respectivamente, das $N$ partículas no espaço de fase; $h_{P}$ é a constante de Planck e $k_{\mathrm{B}}$ é a constante de Boltzmann.

Suponha que tenha sido gerada uma série de configurações de duas alturas consecutivas $2 h_{i}$ e $2 h_{i+1}$ utilizando o método de Monte Carlo com algoritmo de Metropolis. Nesse caso, o hamiltoniano não possui o termo de energia cinética e se torna dependente somente das posições das partículas do sistema: $\mathcal{H}(\mathbf{p}, \mathbf{r})=U(\mathbf{r})$. A diferença de energia livre $\Delta \mathcal{F}$ entre os estados $2 h_{i}$ e $2 h_{i+1}$ é dada por:

$$
\begin{gathered}
\Delta \mathcal{F}=-k_{\mathrm{B}} T \ln \left(\frac{\mathcal{Z}_{i}}{\mathcal{Z}_{i+1}}\right) \\
\Delta \mathcal{F}=-k_{\mathrm{B}} T \ln \left(\frac{\int \exp \left\{\frac{-U_{i}(\mathbf{r})}{k_{\mathrm{B}} T}\right\} d \mathbf{r}}{\int \exp \left\{\frac{-U_{i+1}(\mathbf{r})}{k_{\mathrm{B}} T}\right\} d \mathbf{r}}\right) .
\end{gathered}
$$

Seja $\Delta U=U_{i}(\mathbf{r})-U_{i+1}(\mathbf{r})$ a diferença de energia interna entre as configurações das alturas $2 h_{i}$ e $2 h_{i+1}$. Com essas informações, pode-se calcular a probabilidade de se obter uma diferença de energia potencial $\Delta U$ no espaço 
de configurações de $2 h_{i}$, denominada $P_{i}(\Delta U)$. No ensemble canônico, essa probabilidade $P_{i}(\Delta U)$ é dada por $[52,54,55]$ :

$$
P_{i}(\Delta U)=\frac{\int \exp \left\{-\frac{U_{i}(\mathbf{r})}{k_{\mathrm{B}} T}\right\} \delta\left(U_{i}(\mathbf{r})-U_{i+1}(\mathbf{r})-\Delta U\right) d \mathbf{r}}{\mathcal{Z}_{i}}
$$

Fazendo a substituição de $U_{i}(\mathbf{r})=\Delta U+U_{i+1}(\mathbf{r})$ na exponencial da Eq. 2.24, pode-se obter:

$$
P_{i}(\Delta U)=\frac{\int \exp \left\{-\frac{U_{i+1}(\mathbf{r})+\Delta U}{k_{\mathrm{B}} T}\right\} \delta\left(U_{i}(\mathbf{r})-U_{i+1}(\mathbf{r})-\Delta U\right) d \mathbf{r}}{\mathcal{Z}_{i}},
$$

que expandindo gera:

$P_{i}(\Delta U)=\frac{\mathcal{Z}_{i+1}}{\mathcal{Z}_{i}} \exp \left\{-\frac{\Delta U}{k_{\mathrm{B}} T}\right\} \frac{\int \exp \left\{\frac{-U_{i+1}(\mathbf{r})}{k_{\mathrm{B}} T}\right\} \delta\left(U_{i}(\mathbf{r})-U_{i+1}(\mathbf{r})-\Delta U\right) d \mathbf{r}}{\mathcal{Z}_{i+1}}$.

Sendo $P_{i+1}(\Delta U)$, a probabilidade de obter uma diferença de energia potencial $\Delta U$ no espaço de configurações da altura $2 h_{i+1}$, pode-se reescrever a Eq. 2.24 para $2 h_{i+1}$. Assim:

$$
P_{i+1}(\Delta U)=\frac{\int \exp \left\{\frac{-U_{i+1}(\mathbf{r})}{k_{\mathrm{B}} T}\right\} \delta\left(U_{i}(\mathbf{r})-U_{i+1}(\mathbf{r})-\Delta U\right) d \mathbf{r}}{\mathcal{Z}_{i+1}} .
$$

Substituindo a Eq. 2.26 na Eq. 2.25 obtem-se: 


$$
P_{i}(\Delta U)=\frac{\mathcal{Z}_{i+1}}{\mathcal{Z}_{i}} \exp \left\{\frac{-\Delta U}{k_{\mathrm{B}} T}\right\} P_{i+1}(\Delta U)
$$

Calculando o logaritmo da Eq. 2.27 e substituindo a Eq. 2.23 obtem-se:

$$
\begin{gathered}
\ln \left(P_{i}(\Delta U)\right)=\ln \left(\frac{\mathcal{Z}_{i+1}}{\mathcal{Z}_{i}} \exp \left\{\frac{-\Delta U}{k_{\mathrm{B}} T}\right\} P_{i+1}(\Delta U)\right) \\
\ln \left(P_{i}(\Delta U)\right)=\ln \left(\frac{\mathcal{Z}_{i+1}}{\mathcal{Z}_{i}}\right)-\frac{\Delta U}{k_{\mathrm{B}} T}+\ln \left(P_{i+1}(\Delta U)\right) \\
\ln \left(P_{i}(\Delta U)\right)=\frac{\Delta \mathcal{F}-\Delta U}{k_{\mathrm{B}} T}+\ln \left(P_{i+1}(\Delta U)\right) .
\end{gathered}
$$

Isolando $\Delta \mathcal{F}$ em um dos lados, obtem-se:

$$
\Delta \mathcal{F}=k_{\mathrm{B}} T\left(\left(\ln \left(P_{i+1}(\Delta U)\right)-\frac{\Delta U}{k_{\mathrm{B}} T}\right)-\ln \left(P_{i}(\Delta U)\right)\right)
$$

Assim, a diferença de energia livre $\Delta \mathcal{F}$ entre duas alturas consecutivas pode ser obtida pela diferença de um ajuste de cada um dos termos do lado direito da Eq. 2.29, que podem ser ajustados por um polinômio. Esses polinômios serão iguais entre si a menos de uma constante. O valor desta constante corresponde ao valor da energia livre.

Um outro modo de obter a diferença de energia livre $\Delta \mathcal{F}$ provem da integração em $\Delta U$ da Eq. 2.27. Multiplicando ambos os lados da Eq.2.23 pela $\exp \{\}$ e substituindo o resultado na Eq. 2.27: 


$$
P_{i}(\Delta U)=\exp \left\{\frac{\Delta \mathcal{F}-\Delta U}{k_{\mathrm{B}} T}\right\} P_{i+1}(\Delta U)
$$

Sendo $P_{i}(\Delta U)$ e $P_{i+1}(\Delta U)$ distribuições normalizadas, ou seja:

$$
\int_{-\infty}^{+\infty} P_{i}(\Delta U) \mathrm{d}(\Delta U)=1
$$

e

$$
\int_{-\infty}^{+\infty} P_{i+1}(\Delta U) \mathrm{d}(\Delta U)=1
$$

Integrando-se a Eq. $2.30 \mathrm{em} \Delta U$ :

$$
\begin{gathered}
\int_{-\infty}^{+\infty} P_{i}(\Delta U) \mathrm{d}(\Delta U)=\int_{-\infty}^{+\infty} \exp \left\{\frac{\Delta \mathcal{F}-\Delta U}{k_{\mathrm{B}} T}\right\} P_{i+1}(\Delta U) \mathrm{d}(\Delta U) \\
1=\exp \left\{\frac{\Delta \mathcal{F}}{k_{\mathrm{B}} T}\right\} \int_{-\infty}^{+\infty} \exp \left\{-\frac{\Delta U}{k_{\mathrm{B}} T}\right\} P_{i+1}(\Delta U) \mathrm{d}(\Delta U)
\end{gathered}
$$

ou seja,

$$
\Delta \mathcal{F}=-k_{\mathrm{B}} T \ln \left(\int_{-\infty}^{+\infty} \exp \left\{-\frac{\Delta U}{k_{\mathrm{B}} T}\right\} P_{i+1}(\Delta U) \mathrm{d}(\Delta U)\right)
$$

As Equações 2.29 e 2.32 podem ser utilizadas para encontrar numericamente a energia livre no método de Monte Carlo. Nessa dissertação vários métodos foram testados, todavia a Eq 2.29 mostrou uma estabilidade numérica maior. No capitulo de Resultados serão apresentado os cálculos numéricos utilizando um método baseado na Eq. 2.29. 


\section{Capítulo 3}

\section{Resultados para o Modelo}

\section{Macroscópico da Ponte Líquida}

Realizaram-se dois estudos com esse modelo de gás de rede para pontes líquidas macroscópicas. O primeiro estudo foi dedicado ao processo de formação da ponte líquida inspirado em um problema da fisiologia pulmonar: a emissão de um ruído de crepitação durante a formação da ponte líquida que foi observado experimentalmente por Vysheskiy e colaboradores [56].

O outro estudo foi dedicado às propriedades termodinâmicas de uma rede simplificada do modelo, ou seja, sem utilizar as placas planas. Estudou-se as transições de fase alterando a temperatura e a densidade. Também foi estudado o efeito de escala alterando o tamanho do sistema mas mantendo a concentração de partículas de líquido constante. Outro estudo foi para entender os efeitos das interações de longo alcance. Além disso, a energia livre do modelo foi estimada utilizando o método "Overlapping Distribution". 


\subsection{Estudo da Formação}

Recentemente, Vysheskiy e colaboradores [56] examinaram sistematicamente a conexão entre os ruídos de crepitação de inspiração e o incomum ruído de crepitação de expiração, cujo mecanismo de geração do som não é trivial. Durante a expiração, pontes líquidas se formam no interior das vias aéreas. A diminuição da energia livre da superfície devido à formação da ponte líquida foi aqui estudada e fez-se a hipótese que a diminuição dessa energia está relacionada com esses ruídos de crepitação. A seguir serão discutidos e apresentados os resultados obtidos.

\subsubsection{Diferença de Energia Livre de uma Gota e da Ponte Líquida}

A diferença de energia livre $\Delta \mathcal{F}$ de uma ponte líquida e de uma gota foi calculada para diferentes ângulos de contato $\Theta$. Na Figura 3.1 pode-se observar a comparação dos resultados numéricos e analíticos da energia livre $\mathcal{F}_{b}\left(\Omega, h_{\min }\right)$ da ponte líquida na altura mínima para sua formação $2 h_{\min }$, calculado a partir da Eq. 2.11, e a energia livre de uma única gota $\mathcal{F}_{0,1}(\Omega)$ presa na placa utilizando a Eq.2.12. Para o modelo numérico utilizou-se apenas a energia interna $U$ calculada a partir da Eq.2.17 e considerando apenas o agregado que forma a ponte líquida e a gota. Para qualquer ângulo de contato, a energia livre da ponte líquida é menor que a energia livre da gota. Assim, no momento em que a gota encosta na placa uma ponte líquida será formada, e a perda dessa energia pode ser dissipada na forma de energia sonora. 


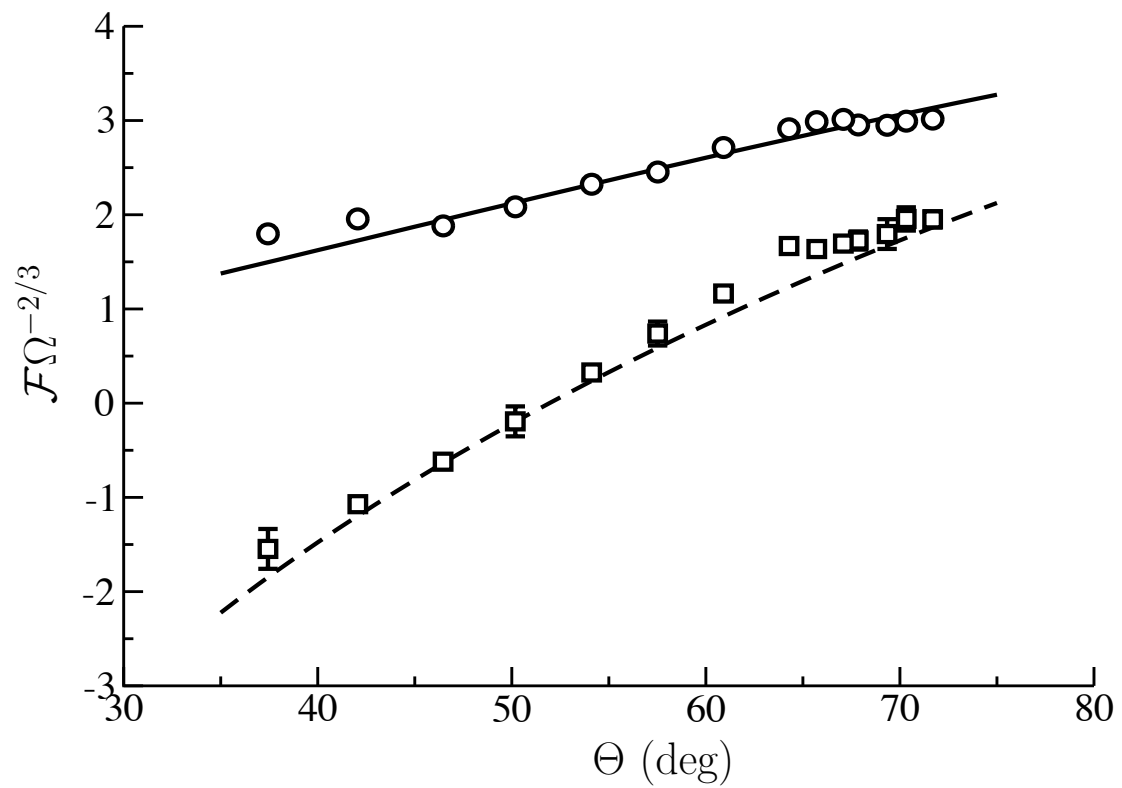

Figura 3.1: Energia livre em função do ângulo de contato $\Theta$, no momento da formação da ponte líquida. A linha contínua é a solução analítica para o máximo de energia livre da gota (Eq.2.12) e os círculos abertos são os respectivos resultados numéricos. A linha tracejada é a solução analítica para o máximo da energia livre da ponte líquida (Eq. 2.11) e os quadrados abertos são os respectivos resultados numéricos.

\subsubsection{O Efeito da Interação de Longo Alcance}

Durante o estudo da formação da ponte líquida verificou-se um efeito de tamanho finito no modelo de gás de rede para pontes líquidas macroscópicas. De acordo com o cálculo analítico, a ponte líquida será formada se a separação entre os planos for igual ou menor que a distância mínima $2 h_{\min , 1}$ para uma gota e $2 h_{\min , 2}$ para duas gotas, obtidos respectivamente das equações Eq. 2.7 e Eq. 2.8. Entretanto, como pode ser verificado na Fig. 3.2, a ponte líquida sempre se formará em uma outra altura $2 h_{\text {min,c }}$ localizada entre $2 h_{\min , 1}$ e $2 h_{\min , 2}$. Essa condição é válida para todos os valores de $\Theta$ testados. 


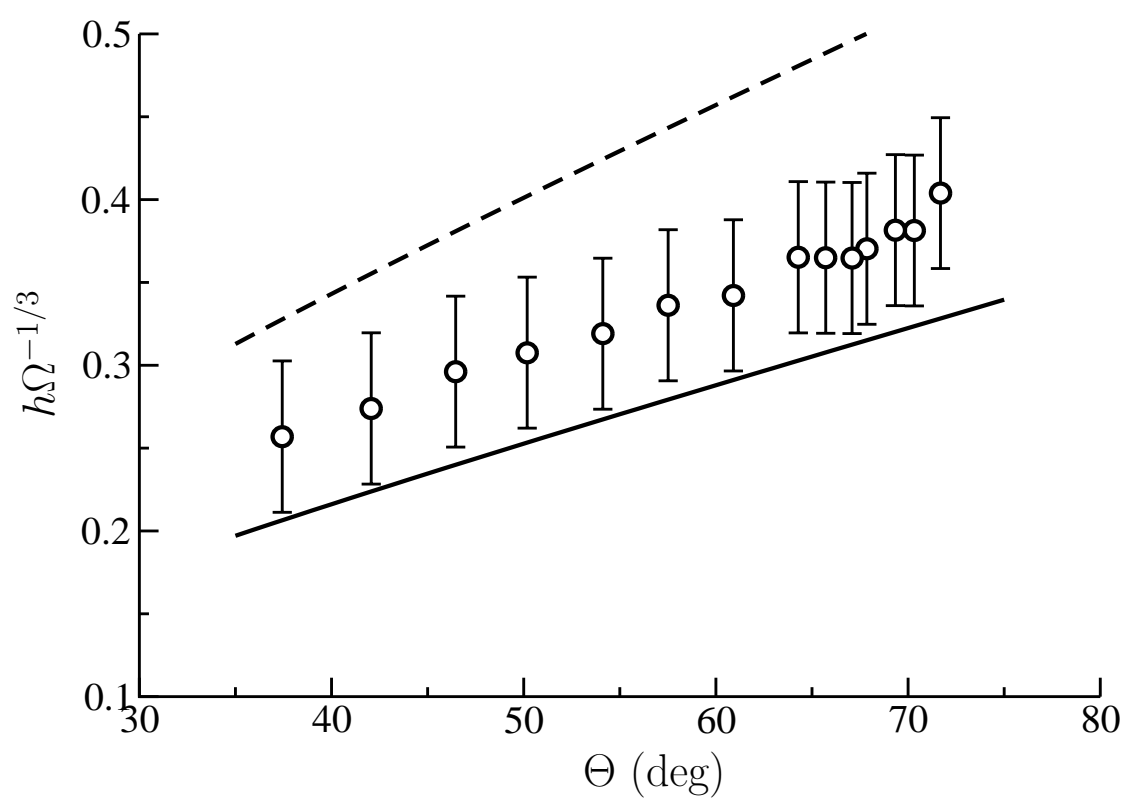

Figura 3.2: Distância mínima entre os dois planos na qual as gotas são estáveis. A linha sólida é o resultado do cálculo analítico para uma gota utilizando a Eq. 2.7; a linha tracejada é o resultado analítico para duas gotas utilizando a Eq. 2.8. Os círculos indicam o valor de $2 h_{\text {min,c }}$ para o qual a ponte se forma efetivamente durante a simulação numérica. As barras de erro verticais correspondem à resolução de um sítio da rede para a distância na qual a ruptura é observada.

De acordo com a Fig. 3.2, a formação da ponte líquida sempre acontece antes da posição mínima $2 h_{\min , 1}$ previsto do cálculo analítico. Devido ao efeito de temperatura, haverá sempre partículas de líquido na superfície dos dois planos e no "bulk", formando o vapor. Quando a gota presa em um dos planos ficar três sítios de distância do plano oposto, o vapor nessa região de separação vai gerar uma instabilidade atraindo mais vapor. Consequentemente, será iniciada uma avalanche que irá levar à formação da ponte líquida, como ilustrado na Fig. 3.3. Este efeito de vapor é muito forte e a maioria das pontes líquidas se formarão neste ponto, o que explica a diferença nos resul- 
tados do cálculo analítico e do modelo do gás de rede para pontes líquidas macroscópicas.
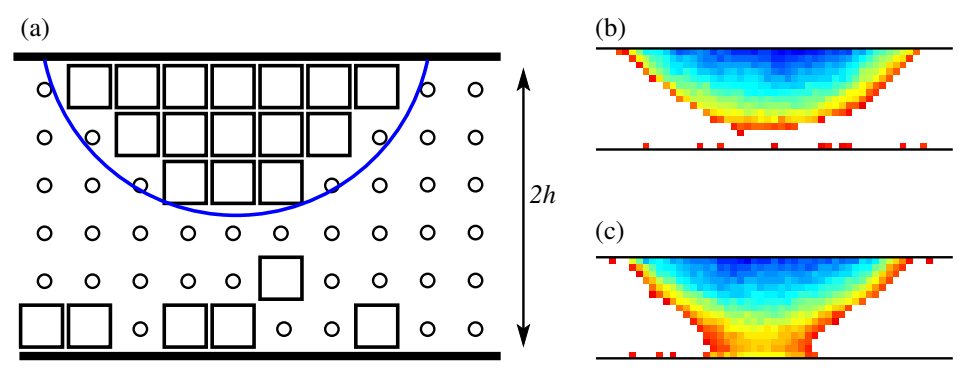

Figura 3.3: Ilustração do modelo instantes antes da formação da ponte líquida. Em (a) as partículas de líquido, gás e parede são representas por quadrados, círculos e uma linha espessa, respectivamente. As partículas de líquidos desconectadas da ponte líquida ou das placas planas são denominadas vapor. Quando as partículas de líquido estão ligadas aos planos, diz-se que estão "molhando" o plano. Todas as demais partículas de líquido formam a gota. Esta ilustração destaca o efeito da presença de vapor entre a gota e o plano "molhado" na parte inferior. Isso pode levar à formação da ponte líquida antes da previsão teórica. Em (b), pode-se observar uma configuração do modelo instantes antes da formação da ponte líquida. O topo da gota está a três sítios de distância do plano inferior "molhado". Em (c), tem-se o instante da formação da ponte líquida.

Então, estudou-se esse efeito de vapor mais detalhadamente para diferentes tamanhos de rede. Todos os parâmetros das interações $J_{i, j}$ foram fixos em um valor que gera um ângulo de contato de aproximadamente $60^{\circ}$. Então a escala do sistema foi variada de forma a manter constante a fração de partículas ar/líquido. Para cada tamanho do sistema no modelo numérico, calculou-se o valor exato de $\Theta$, o valor crítico numérico em que a ponte líquida é formada $2 h_{\min , \mathrm{c}}$ e as soluções analíticas $2 h_{\min , 1}$ e $2 h_{\min , 2}$. Verificou-se que o ângulo de contato $\Theta$ não muda muito para diferentes tamanhos de rede.

Na Figura 3.4 está sendo comparado o valor numérico de $2 h_{\text {min,c }}$ na qual 
a ponte líquida é formada, deslocada de três sítios $\left(2 h=2 h_{\min , \mathrm{c}}-3\right)$ com o resultado analítico $2 h_{\min , 1}$ em função do tamanho do sistema. As barras de erros representam o tamanho de um sítio da rede normalizada pelo volume da ponte líquida $\Omega^{1 / 3}$. Pode-se observar que o erro decresce com o tamanho do sistema. À medida que o tamanho do sistema aumenta, o valor numérico converge para o valor analítico esperado.

No trabalho de Alencar e colaboradores sobre o processo da ruptura da ponte líquida [34] não foi encontrado nenhum efeito de tamanho finito pois a ponte líquida já está formada e todo o sistema é praticamente contínuo. Logo, um único sítio tem um efeito desprezível, ver Fig. 3.3c. Entretanto, durante o processo de formação da ponte líquida, foi encontrado esse efeito de interação de longo alcance, aqui três sítios, que pode ser desprezado para sistemas muito grandes. Em outras palavras, uma partícula de vapor entre a gota e a placa pode levar a formação da ponte líquida, o que justifica $2 h_{\text {min,c }}$ ser maior que $2 h_{\min , 1}$, ver Fig. 3.3b. 


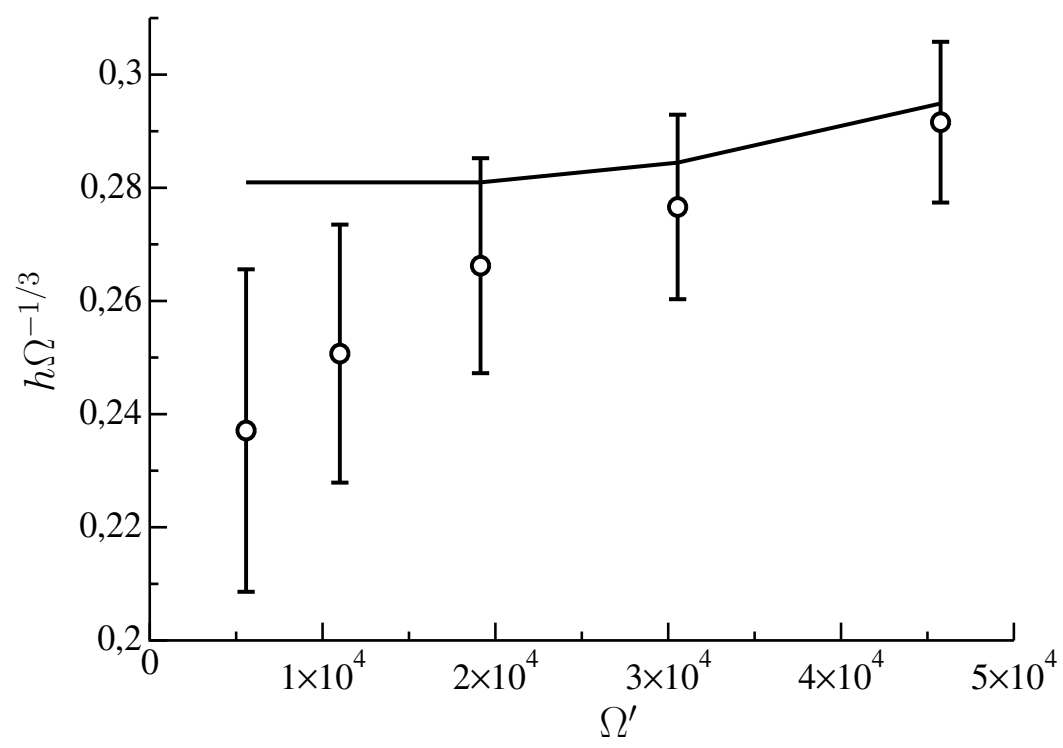

Figura 3.4: Análise da altura mínima crítica $2 h_{\text {min,c }}$ para a formação da ponte líquida utilizando cinco tamanhos de redes cúbicas diferentes e mantendo a fração de partículas de líquido/gás constante. O ângulo de contato foi mantido em aproximadamente $\Theta \approx 60^{\circ}$. As dimensões das redes utilizadas foram 40, 50, 60, 70 e 80, com os respectivos números total de partículas de líquido $\Omega^{\prime}$ : 5574, 11000, 19140, 30544 e 45760. A linha contínua é o resultado analítico para a altura mínima da formação da ponte líquida $2 h_{\min , 1}$, normalizado pelo volume da ponte líquida $\Omega^{1 / 3}$. Esse cálculo é baseado nas médias do volume e médias do ângulo de contato $\Theta$ para 30 simulações. Podese observar que o cálculo analítico não é constante, pois o ângulo de contato e a razão $\Omega / \Omega^{\prime}$ alteram com o tamanho da rede. Os círculos são as meias distâncias da altura crítica para formação da ponte líquida normalizadas $2 h_{\text {min,c }} \Omega^{-1 / 3}$ e subtraída de três sítios da rede para cada tamanho de rede. As barras de erro, acima e abaixo do ponto médio, representam o tamanho normalizado de um sítio da rede. 


\subsubsection{Histerese na Energia Livre}

Na Figura 3.5 foram comparadas as simulações para quando há formação da ponte líquida e quando há ruptura. Pode-se observar uma curva de histerese com duas descontinuidades da energia livre. Isso indica um possível mecanismo para a geração dos ruídos de crepitação gerados no pulmão durante o processo de inspiração e expiração $[13,56]$.

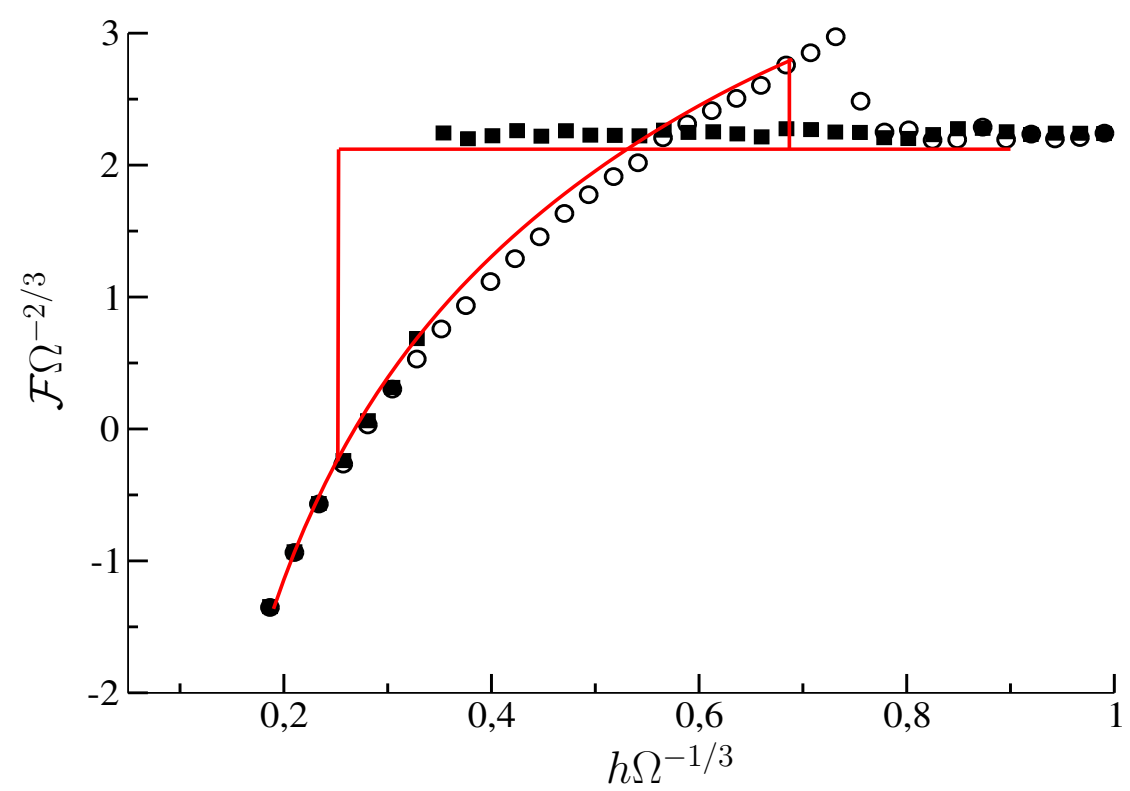

Figura 3.5: Energia livre total normalizada pelo volume da ponte líquida $\mathcal{F} \Omega^{-2 / 3}$ em função da meia distância normalizada $h$ durante a formação da ponte líquida (quadrados) e da ruptura da ponte líquida (círculos). A linha vertical tracejada representa $2 h_{\mathrm{c}}=0,687$ e $2 h_{\min , 1}=0,247$ obtidos analiticamente [34]. As linhas contínuas são os cálculos analíticos da normalização energia livre total $\mathcal{F}$ para a ponte líquida e para a gota. Nestas simulações foram utilizados $\Theta \approx 50^{\circ}, 10000$ partículas de líquido e tamanho de rede de $60 \times 60 \times 52$. A histerese na energia livre destaca duas regiões de instabilidade, quando a ponte líquida é esticada e quando a gota é aproximada da placa plana. Cada ponto na curva é uma média de 40000 passos de Monte Carlo. 


\subsubsection{Força Exercida na Placa}

Foi realizado um estudo sobre a força que a ponte líquida exerce nas placas planas. A contínua diminuição da energia livre à medida que a distância entre as placas $2 h$ diminui irá gerar uma força atrativa entre as mesmas [43]. A movimentação dos planos é quase-estática, ou seja, sempre que uma das placas move o sistema espera atingir o equilíbrio antes de uma nova movimentação entre as placas planas. Existe uma força externa $F_{e}$, que é igual à força resultante devido à tensão superficial da ponte líquida que pode ser calculada a partir do gradiente de energia:

$$
F_{e}=-\frac{1}{2} \frac{\partial U}{\partial h}
$$

Na Figura 3.6, é apresentado $F_{e}$ calculada numericamente da energia do agregado que forma a ponte líquida conforme Eq.3.1, normalizada pelo volume da ponte líquida para três ângulos de contato $\Theta=39^{\circ}, 50^{\circ}$ e $66^{\circ}$. Os resultados obtidos são análogos aos resultados analíticos obtidos para os mesmos ângulos de contato, aplicando a Eq.2.11 na Eq.3.1. Pode-se notar que a força aumenta à medida que $2 h$ diminui. Isso irá levar ao colapso entre os planos como observado em tubos flexíveis. Uma via aérea real irá se manter colapsada até que a força no tecido para reabrir a via-aérea seja igual à força da tensão superficial. Pode-se interpretar a força calculada, apresentada na Fig. 3.6, como a força necessária para reabrir a via aérea. Essa força é muito grande para pequenas distâncias $2 h$, e diminui à medida que $2 h$ aumenta. Portanto, uma vez que a força do plano seja maior que a força necessária para manter o plano estável, a placa irá mover, quebrando a ponte liquida 
muito rapidamente devido ao perfil da força em função de $2 h$.

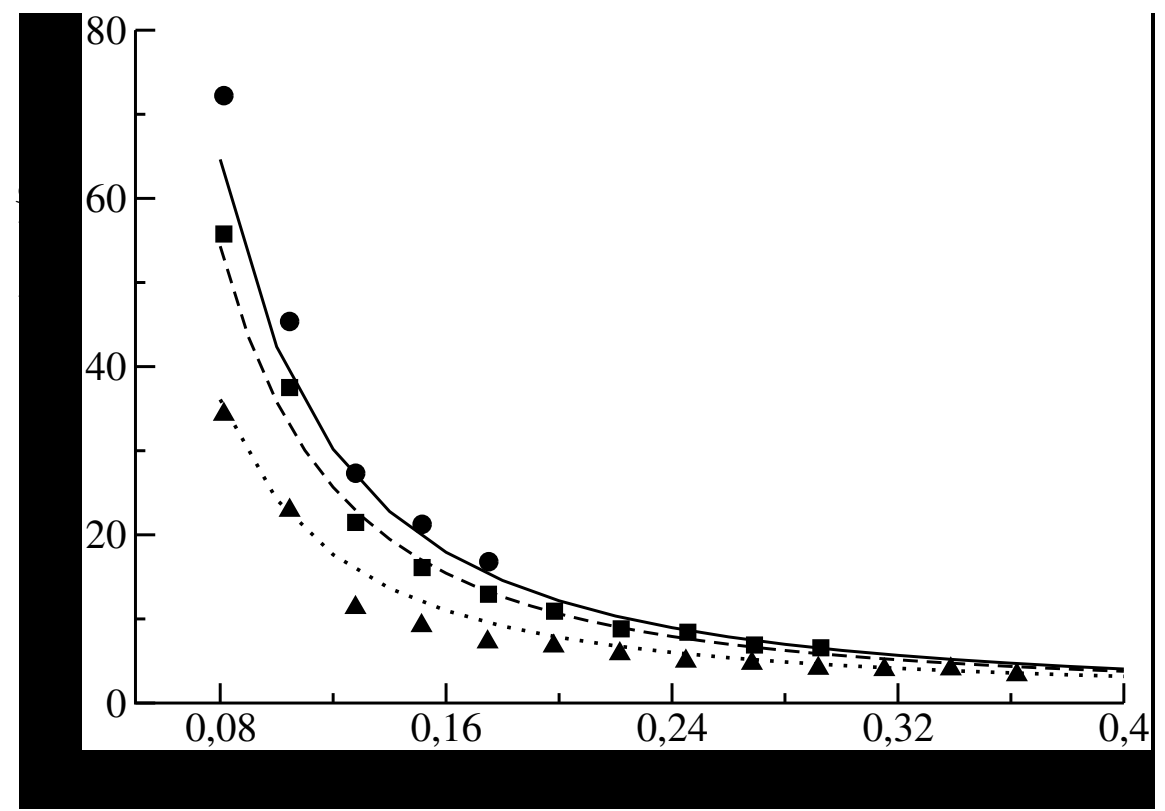

Figura 3.6: Força entre as planos para três diferentes ângulos de contato $\Theta$ calculados usando a Eq.(3.1). Circulo, quadrado e triângulo representam $\Theta$ igual a $39^{\circ}, 50^{\circ}$ e $66^{\circ}$, respectivamente. As linhas (-) das cores preta, azul e vermelha representam, respectivamente, os cálculos analíticos dos ângulos $\Theta$ igual a $39^{\circ}, 50^{\circ}$ e $66^{\circ}$. Utilizou-se uma rede de tamanho $60 \times 60 \times 52$ e com 10000 partículas de líquido. 


\subsection{Analise Termodinâmica do Modelo}

Para realizar o estudo termodinâmico do fluido, utilizou-se uma versão simplificada do modelo, ou seja, sem as placas planas. Estudou-se somente o modelo de gás de rede de partículas microscópicas com interações nas distâncias de $1, \sqrt{2}$ e $\sqrt{3}$.

Em uma etapa inicial, realizou-se o cálculo analítico desse modelo em uma rede de três dimensões de lado " $L$ " com duas partículas de líquido $(l)$ e $\left(L^{3}-2\right)$ partículas de gás $(g)$. A função de partição foi calculada e a partir desta calculou-se a energia interna e o calor específico. Estas equações analíticas foram comparadas com resultados das simulações.

No segundo estudo, analisou-se o efeito da escala do sistema mantendo sempre a mesma concentração de partículas. Foi estudado como a energia do agregado é alterada para $T=0 \mathrm{~J} / k_{\mathrm{B}}$.

No terceiro estudo, analisou-se a transição de fase do sistema. A temperatura crítica foi determinada. A curva de transição de fase também foi determinada.

Fez-se também o cálculo da força exercida na placa devido a ponte líquida para diferentes temperaturas. Numa primeira analise foi considerado somente a energia interna do sistema e depois foi considerado a energia livre. Para isto, foi utilizado o método de "Overlapping Distribution".

Finalmente, discutiu-se uma possível interpretação física para o modelo de gás de rede na escala macroscópica. 


\subsubsection{Sistema com Duas Partículas do Líquido}

Quando duas partículas de líquido estão interagindo através dos primeiros vizinhos, ou seja, as partículas estão separadas por uma distância de 1, a energia é dada por:

$$
E_{1}=\left(J_{l l}^{1} \times 1\right)+\left(J_{g l}^{1} \times 10\right)=(-1 \times 1)+(-0,02 \times 10)=-1,2 J,
$$

em que o primeiro termo representa a interação entre as partículas de líquido e o segundo termo a interação entre as partículas de líquido com as partículas de gás. Se as partículas de líquido estão interagindo via os segundos vizinhos, distância de $\sqrt{2}$, e seguindo a mesma ordem dos termos, a energia é dada por:

$$
E_{\sqrt{2}}=\left(J_{l l}^{2} \times 1\right)+\left(J_{g l}^{1} \times 12\right)=(-0,7 \times 1)+(-0,02 \times 12)=-0,94 J .
$$

Para terceiros vizinhos, distância de $\sqrt{3}$, a energia é dada por:

$$
E_{\sqrt{3}}=\left(J_{l l}^{3} \times 1\right)+\left(J_{g l}^{1} \times 12\right)=(-0,4 \times 1)+(-0,02 \times 12)=-0,64 J .
$$

Finalmente, quando as partículas de líquido estão separadas por uma distância maior que o terceiro vizinho, não existirá interação líquido-líquido e a energia é dada por:

$$
E_{\text {sep }}=J_{g l}^{1} \times 12=-0,02 \times 12=-0,24 J \text {. }
$$

Para cada uma dessas energias, determinou-se o número de configurações 
existentes considerando a condição periódica de contorno no modelo. Como exemplo, será realizado o cálculo do número de configurações possíveis para duas partículas de líquido que interagem via primeiros vizinhos, ver Fig.3.7.

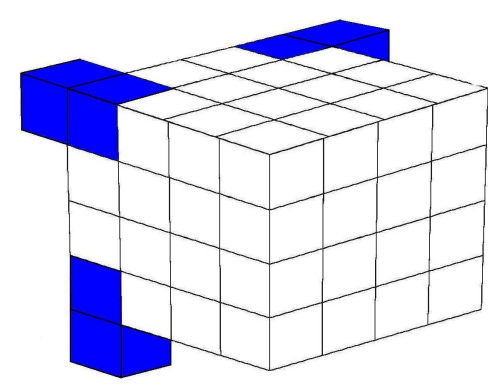

Figura 3.7: Ilustração da condição de contorno periódica utilizada no cálculo das configurações para a situação de duas partículas interagindo via primeiros vizinhos.

Deve-se calcular quantas configurações existem na rede quando se rotaciona e translada essas duas partículas ligadas via primeiros vizinhos. Como existem seis sítios na distância 1 , existem $6 / 2=3$ maneiras de combinar essas duas partículas, pois não existe no modelo troca de partículas iguais. Essas três maneiras contemplam a rotação. Agora, considerando o fato dessas duas partículas poderem se transladar na rede, existem $L \times L \times L$ maneiras, já considerando a condição periódica de contorno. Assim, o número total de configurações possíveis é de $3 \times L^{3}$.

O raciocínio é análogo para as partículas interagindo via segundos e terceiros vizinhos. No caso de segundo vizinho, as partículas possuem 12/2 =6 maneiras de se combinar. Isto resulta, após as translações, num total de $6 \times L^{3}$ configurações totais do sistema. Analogamente, para terceiros vizinhos, as partículas de líquido podem se combinar de $8 / 2=4$ maneiras resultando num total de $4 \times L^{3}$. O número de configurações, para as partículas separadas por 
uma distância maior que terceiro vizinho, é o número de configurações totais menos o número de configurações das partículas interagindo via primeiros, segundos e terceiros vizinhos.

Com esses resultados calculou-se as probabilidades $P_{1}, P_{\sqrt{2}}, P_{\sqrt{3}}$ e $P_{\text {sep }}$ de se obter uma configuração para cada energia. O número total de configurações possíveis é dado pela permutação do número total de partículas no sistema $L^{3}$ ! dividido pelo número de permutações das partículas iguais: 2! para o caso das partículas de líquido, e $\left(L^{3}-2\right)$ ! para o caso das partículas de gás. Assim, a probabilidade de obter as partículas juntas via interações de primeiros, segundos e terceiros vizinhos, e sem interagir em uma rede de lado $L$ são dadas por:

$$
\begin{gathered}
P_{1}=\frac{3 \times L^{3}}{\frac{L^{3} !}{2 ! \times\left(L^{3}-2\right) !}}=\frac{6}{L^{3}-1} \\
P_{\sqrt{2}}=\frac{12}{L^{3}-1} \\
P_{\sqrt{3}}=\frac{8}{L^{3}-1} \\
P_{\text {sep }}=1-P_{1}-P_{\sqrt{2}}-P_{\sqrt{2}}
\end{gathered}
$$

Considerando $L=4$ pode-se obter: $P_{1} \approx 0,09523, P_{\sqrt{2}} \approx 0,1904, P_{\sqrt{3}} \approx$ 0,1269 e $P_{\text {sep }} \approx 0,5873$. Realizando uma simulação com 2000000 passos de MC obtive-se os seguintes valores das probabilidades para cada energia: $P_{1} \approx 0,09529, P_{\sqrt{2}} \approx 0,1908, P_{\sqrt{3}} \approx 0,1266$ e $P_{\text {sep }} \approx 0,5871$. 
Agora, é possível calcular as propriedades termodinâmicas desse sistema. Como o modelo foi implementado no ensemble canônico $(N, V, T)$, para calcular as propriedades termodinâmicas, basta calcular a função de partição canônica $\mathcal{Z}$. Assim:

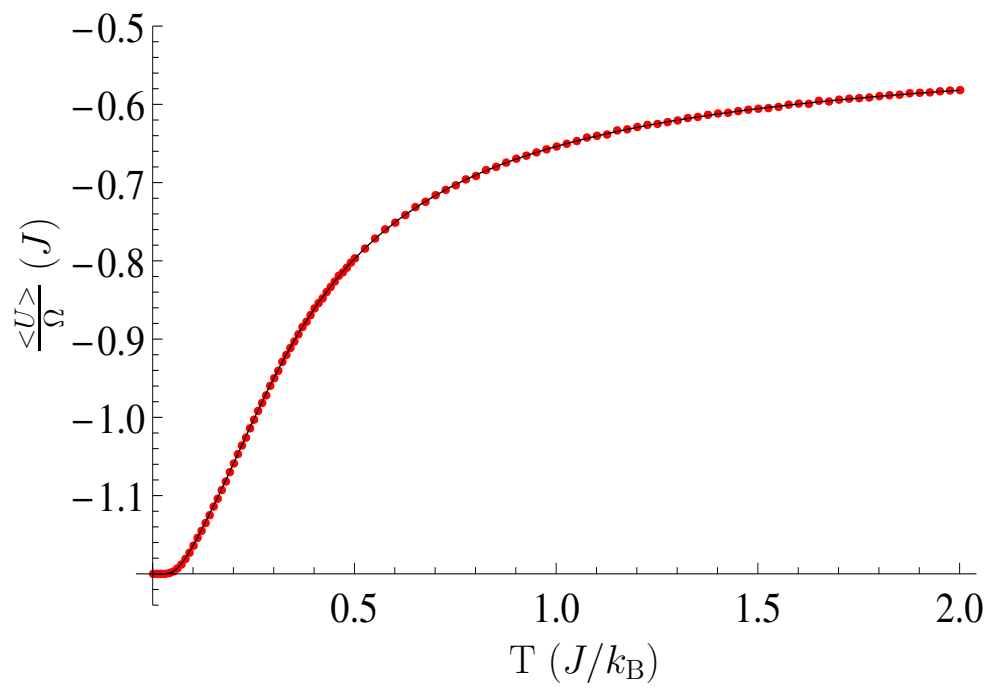

Figura 3.8: Energia interna média por partícula de líquido $\frac{\langle U>}{\Omega}(J)$ em função da temperatura $T\left(J / k_{\mathrm{B}}\right)$. Comparação entre o cálculo analítico dado pela Eq. 3.9 (linha preta) e o resultado numérico do modelo para pontes líquidas macroscópicas (círculos vermelhos) para um sistema contendo somente duas partículas de líquido.

$$
\begin{gathered}
\mathcal{Z}=\sum_{i} e^{\frac{-E_{i}}{k_{\mathrm{B}} T}} \\
\mathcal{Z}=\frac{1}{2} L^{3}\left(\left(L^{3}-27\right) e^{-\frac{E_{\mathrm{sep}}}{T k_{B}}}+6 e^{-\frac{E_{1}}{T k_{B}}}+12 e^{-\frac{E \sqrt{2}}{T k_{B}}}+8 e^{-\frac{E \sqrt{3}}{T k_{B}}}\right) .
\end{gathered}
$$

A energia interna do sistema pode ser escrita como: 


$$
U=-\frac{\partial \ln \mathcal{Z}(\beta))}{\partial \beta} \quad \beta=\frac{1}{k_{\mathrm{B}} T} .
$$

Assim, pode-se obter:

$U(T)=-\frac{-\left(L^{3}-27\right) E_{\mathrm{sep}} e^{-\frac{E_{\mathrm{sep}}}{T k_{B}}}-6 E_{1} e^{-\frac{E_{1}}{T k_{B}}}-12 E_{\sqrt{2}} e^{-\frac{E_{\sqrt{2}}}{T k_{B}}}-8 E_{\sqrt{3}} e^{-\frac{E_{\sqrt{3}}}{T k_{B}}}}{\left(L^{3}-27\right) e^{-\frac{E_{\mathrm{sep}}}{T k_{B}}}+6 e^{-\frac{E_{1}}{T k_{B}}}+12 e^{-\frac{E_{\sqrt{2}}}{T k_{B}}}+8 e^{-\frac{E_{\sqrt{3}}}{T k_{B}}}}$.

O calor específico é obtido como:

$$
c(T)=\frac{\partial U(T)}{\partial T}
$$

Realizou-se simulações com duas partículas de líquido numa rede de lado $L=4$ alterando a temperatura do sistema. Para cada temperatura foram realizados 500000 passos de $\mathrm{MC}$, em que cada ciclo corresponde a tentar trocar todas as partículas de líquido por gás. Os resultados então foram comparados com as equações analíticas. Nos gráficos mostrados na Fig. 3.8 e 3.9 a seguir são apresentados os resultados obtidos do modelo e do cálculo analítico para a energia interna por partícula e para o calor específico por partícula. Esse estudo foi realizado para demostrar a concordância entre o modelo, em uma forma simples, com o esperado analiticamente, validando os cálculos numéricos. 


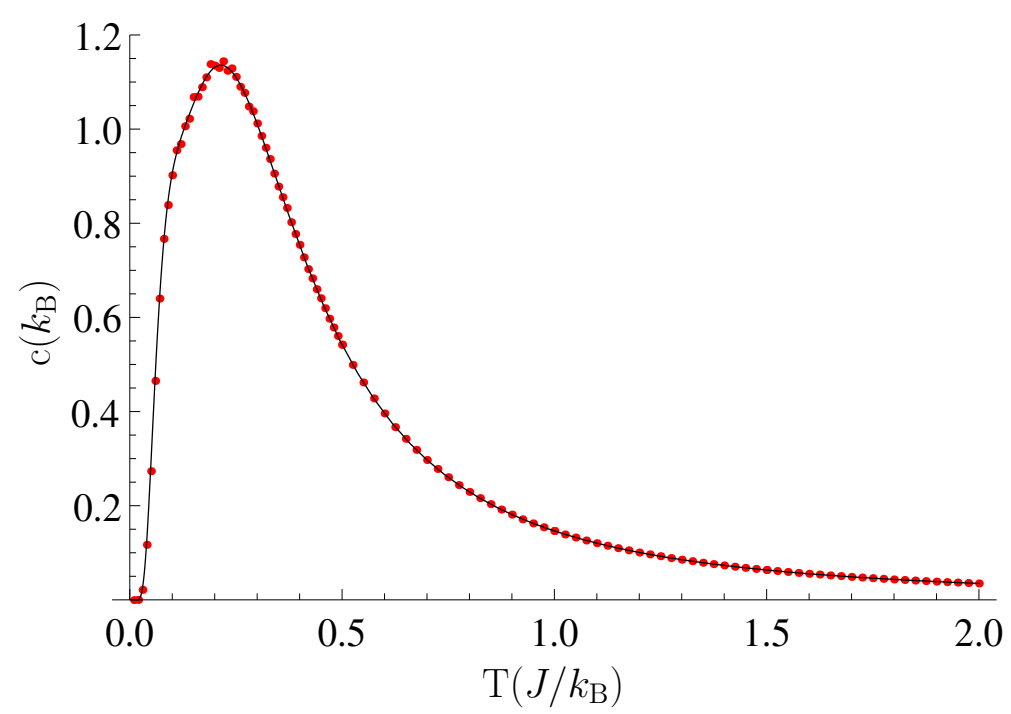

Figura 3.9: Calor específico $c\left(k_{\mathrm{B}}\right)$ em função da temperatura $T\left(J / k_{\mathrm{B}}\right)$. Comparação entre o cálculo analítico dado pela Eq. 3.10(linha preta) e o resultado numérico do modelo para pontes líquidas macroscópicas (círculos vermelhos) para um sistema contendo somente duas partículas de líquido.

\subsubsection{Estudo do Efeito da Escala}

Realizou-se o estudo do efeito da escala do sistema ainda utilizando o modelo simplificado sem as paredes planas. Esse estudo foi realizado na temperatura de $T=0 \mathrm{~J} / k_{\mathrm{B}}$ e a concentração de partículas de líquido em relação às partículas de gás foi mantida constante para diferentes tamanhos do modelo. Esse estudo foi realizado para três valores da energia do potencial de interação das partículas de líquido $J_{l l}$, como apresentados nas Tabelas 2.1, 2.2 e 2.3. Os valores utilizados foram:

1) $J_{l l}^{1}=-0,5 J, J_{l l}^{2}=-0,35 J$ e $J_{l l}^{3}=-0,2 J$;

2) $J_{l l}^{1}=-1,0 \mathrm{~J}, J_{l l}^{2}=-0,7 \mathrm{~J}$ e $J_{l l}^{3}=-0,4 J$;

3) $J_{l l}^{1}=-1,5 J, J_{l l}^{2}=-0,85 J$ e $J_{l l}^{3}=-0,6 J$.

Com esses valores estudou-se como a energia interna por partícula do 


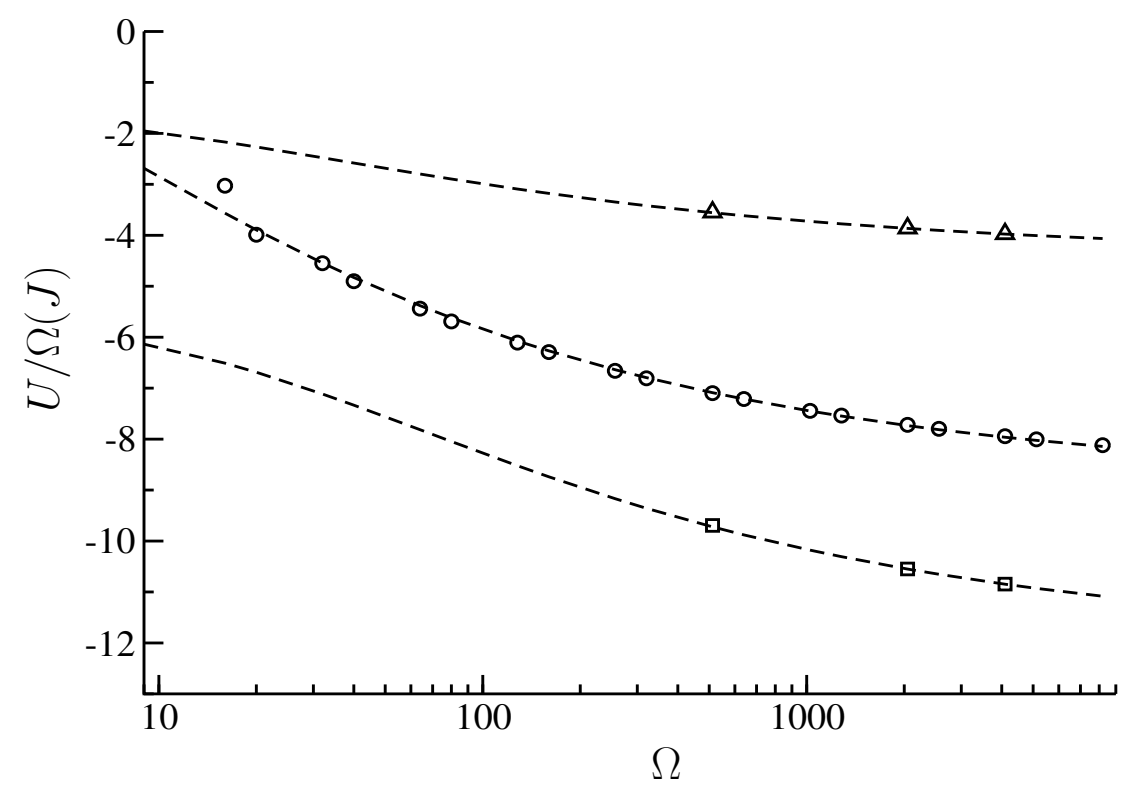

Figura 3.10: Energia interna média por partícula de líquido $U / \Omega(J) \mathrm{em}$ função do número de partículas de líquido $\Omega$ e para três valores da energia potencial de interação das partículas de líquido $J_{l l}$. A proporção de partículas de líquidos e partículas de gás foi mantida constante para os diferentes valores de $\Omega$. Este estudo foi realizado para a temperatura $T=0 \mathrm{~J} / \mathrm{k}_{\mathrm{B}}$.

agregado $U / \Omega$ varia para diferentes volumes de líquidos $\Omega$, ver Fig. 3.10. Encontrou-se uma equação que depende do parâmetro de interação $J_{l l}$ e que ajusta os dados da Fig. 3.10. Essa equação é dada por:

$$
\frac{U}{\Omega}=u_{0}+\frac{B}{\left(v_{e f f}+\Omega\right)^{1 / 3}},
$$

em que $B$ é um parâmetro de ajuste que esta relacionado com a energia da interface e com a relação área/volume do agregado, $u_{0}$ é a energia interna por partícula de uma partícula de líquido cercada por líquidos e é dado por:

$$
u_{0}=\frac{\left(6 \times J_{l l}^{1}\right)+\left(12 \times J_{l l}^{2}\right)+\left(8 \times J_{l l}^{3}\right)}{2} .
$$


A constante $v_{\text {eff }}$ é o volume efetivo de um sítio da rede e é dado por:

$$
v_{e f f}=\left(\frac{\left(6 \times \frac{1}{L_{s}}\right)+\left(12 \times \frac{\sqrt{2}}{L_{s}}\right)+\left(8 \times \frac{\sqrt{3}}{L_{s}}\right)}{26}\right)^{3} V_{s}=2,84 V_{s}
$$

onde $L_{s}$ é o lado de um sítio da rede e $V_{s}$ é o volume de um sítio da rede.

Para cada um daqueles três valores de $J_{l l}$ obteve-se os seguintes valores de $u_{0}$ e $B$ :
1) $u_{0}=-4,4 J$ e $B=6,87$
2) $u_{0}=-8,8 J$ e $B=14,05$;
3) $u_{0}=-12,0 J$ e $B=18,48$.

\subsubsection{Estudo da Transição de Fase do Líquido}

Neste estudo da transição de fase ainda foi utilizado o modelo de pontes líquidas sem as paredes planas. Como já foi discutido na Sec.3.2.1, em que validou-se o modelo computacional comparando os resultados de um sistema de duas partículas com resultados analíticos, nessa parte do estudo montamos um modelo um pouco mais complexo. Aqui a concentração de partículas de líquido é mantida constante, enquanto o tamanho do sistema cresce. Inicialmente, estudou-se como se dá a variação da energia média por partícula de líquido e o calor específico alterando a temperatura de $2 J / k_{\mathrm{B}}$ até $1 J / k_{\mathrm{B}}$ (ver Fig. 3.11 e Fig. 3.12) para 32, 64, 128, 256, 512, 1024, 2048, 4096, 8192 e 16384 partículas de líquido. Note que a medida que o tamanho do sistema cresce, a transição de fase se desloca para a direita e tende a se tornar mais abrupta.

Também foi realizado um estudo em que a energia média por partícula de 


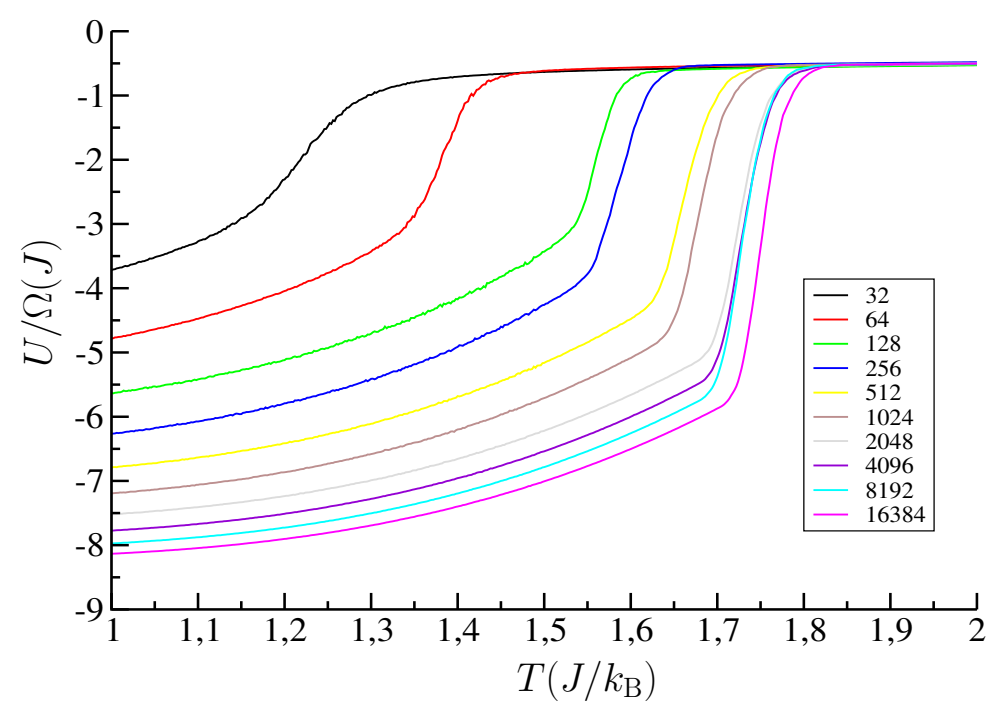

Figura 3.11: Energia interna média por partícula de líquido $U / \Omega(J)$ em função da variação da temperatura $T\left(J / k_{\mathrm{B}}\right)$. A legenda no gráfico mostra o número de partículas de líquido utilizados de forma a manter a concentração de partículas de líquido e gás constante.

líquido era calculada em função da densidade de partícula de líquido, para diferentes valores de temperaturas (ver Fig. 3.13). Uma simulação análoga a esta foi realizada para o modelo de Ising clássico em 3D (ver Fig. 3.14). Diferentemente do modelo de Ising clássico, em que existe simetria entre os os potenciais dos spins $\sigma_{i}=+1$ e $\sigma_{i}=-1$, no modelo aqui desenvolvido essa simetria não existe, ou seja, a situação de densidade zero para líquido é completamente diferente da situação de densidade 1. Isso ocorre devido às energias potenciais de interação escolhidos $\left(J_{i j}^{1}, J_{i j}^{2}\right.$ e $J_{i j}^{3}$, onde $\left.i, j=s, l, g\right)$ entre as partículas. 


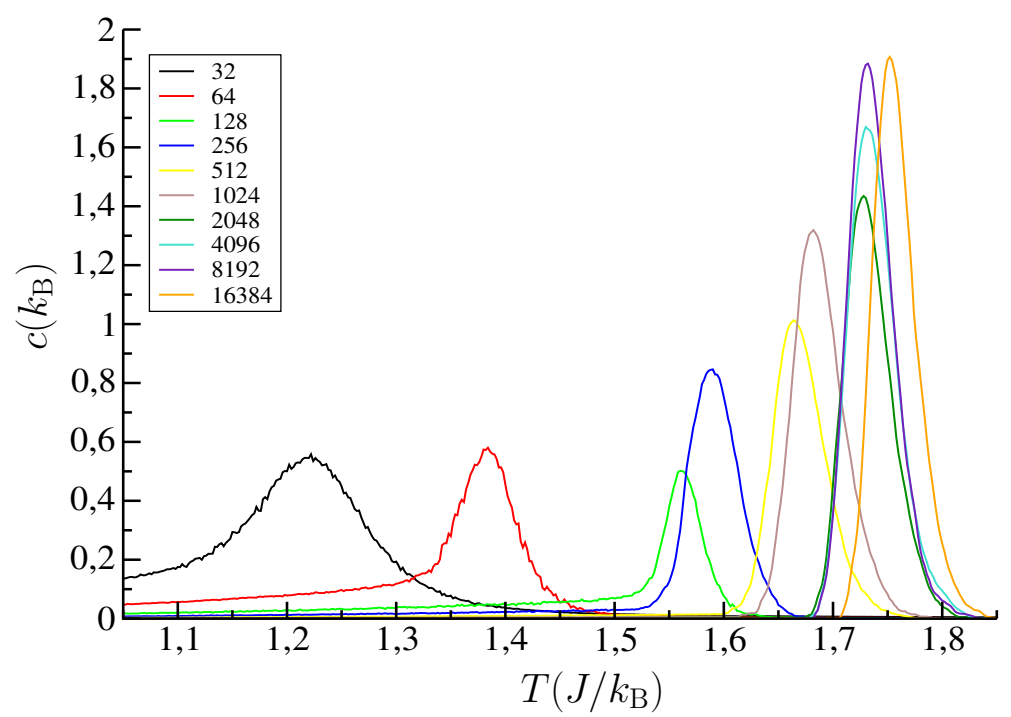

Figura 3.12: Calor específico $c\left(k_{\mathrm{B}}\right)$ em função da variação da temperatura $T\left(J / k_{\mathrm{B}}\right)$. A legenda no gráfico mostra o número de partículas de líquido utilizados de forma a manter a concentração de partículas de líquido e gás constante.

\subsubsection{Calculo da Energia Livre}

Para calcular a energia livre $\mathcal{F}$ e a força, no modelo completo, ou seja, não só contendo as partículas de líquido e ar mas também contendo as paredes planas, o método "Overlaping Distribution", discutido na Sec.2.6, foi utilizado. Como discutido na Sec. 2.6, só é possível calcular a diferença de energia livre entre dois estados, $A$ e $B$. Ou seja, não é possível saber o valor exato da energia livre. No problema em questão, estes estados $A$ e $B$ representam duas alturas consecutivas da ponte líquida $2 h$ e $2 h+1$ e a distribuição de energia para duas alturas consecutivas, como exemplo, está mostrado na Fig. 3.15.

As probabilidades mencionadas nas Eq. 2.24 e 2.26 foram obtidas utilizando o método de Monte Carlo e o algoritmo, calcula a energia livre entre 


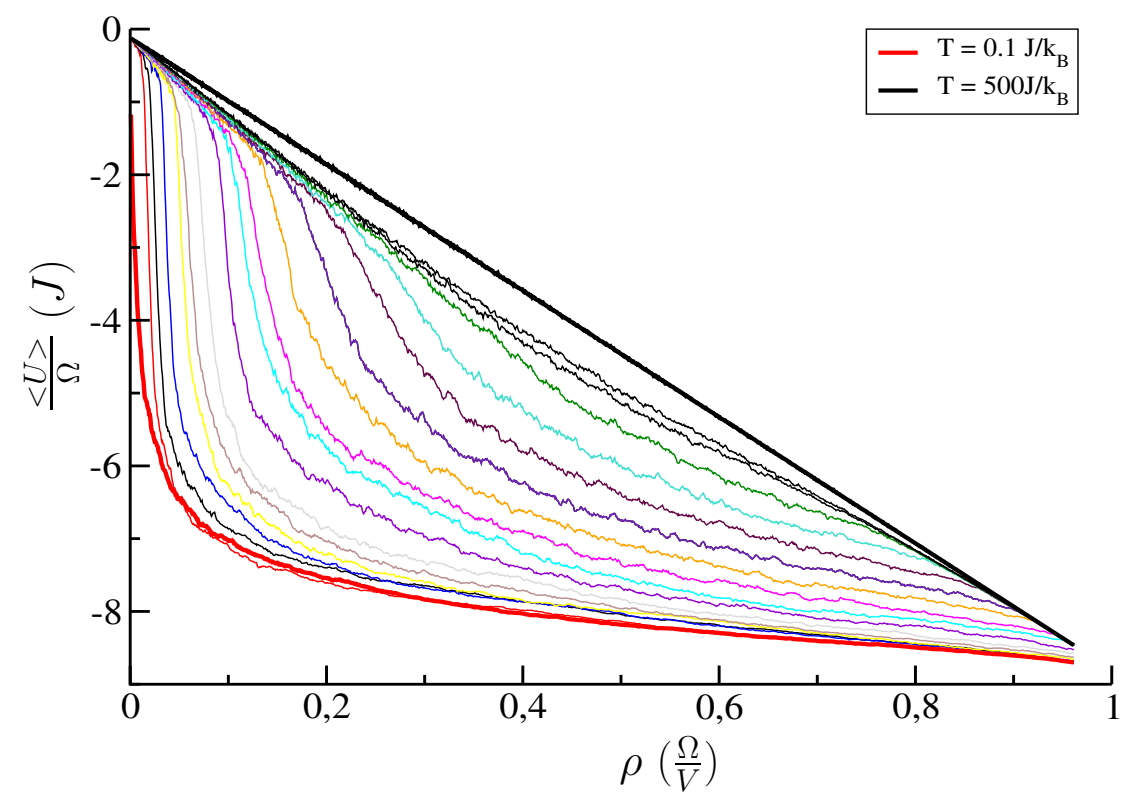

Figura 3.13: Estudo do modelo de gás de rede para pontes líquidas macroscópicas. No gráfico tem-se a energia interna média por partícula de líquido $U / \Omega(J)$ em função da variação da densidade de partículas de líquido $\rho\left(\frac{\Omega}{V}\right)$.

duas alturas consecutivas.

No algoritmo utilizado para calcular $\Delta \mathcal{F}$ foi empregado o método de Monte Carlo discutido na Sec. 2.4. Inicialmente são geradas amostragens da energia das configurações da ponte líquida formada em duas alturas consecutivas: $2 h$ e $2 h+1$. Em seguida, o método de Monte Carlo é aplicado. Faz-se um sorteio de um valor de energia para a amostragem da configuração da altura $2 h$ e da altura $2 h+1$. Então calcula-se a diferença de energia $\Delta E$. Se $\Delta E$ é menor que 0 a configuração é aceita. Caso contrário faz-se o teste da probabilidade, como discutido na Sec.2.4. Pode-se interpretar que se $\Delta E$ é menor que 0 , então a placa troca de posição, ou seja sai da altura $2 h$ para a altura $2 h+1$, e vice versa. A placa pode repetir este processo de troca de al- 


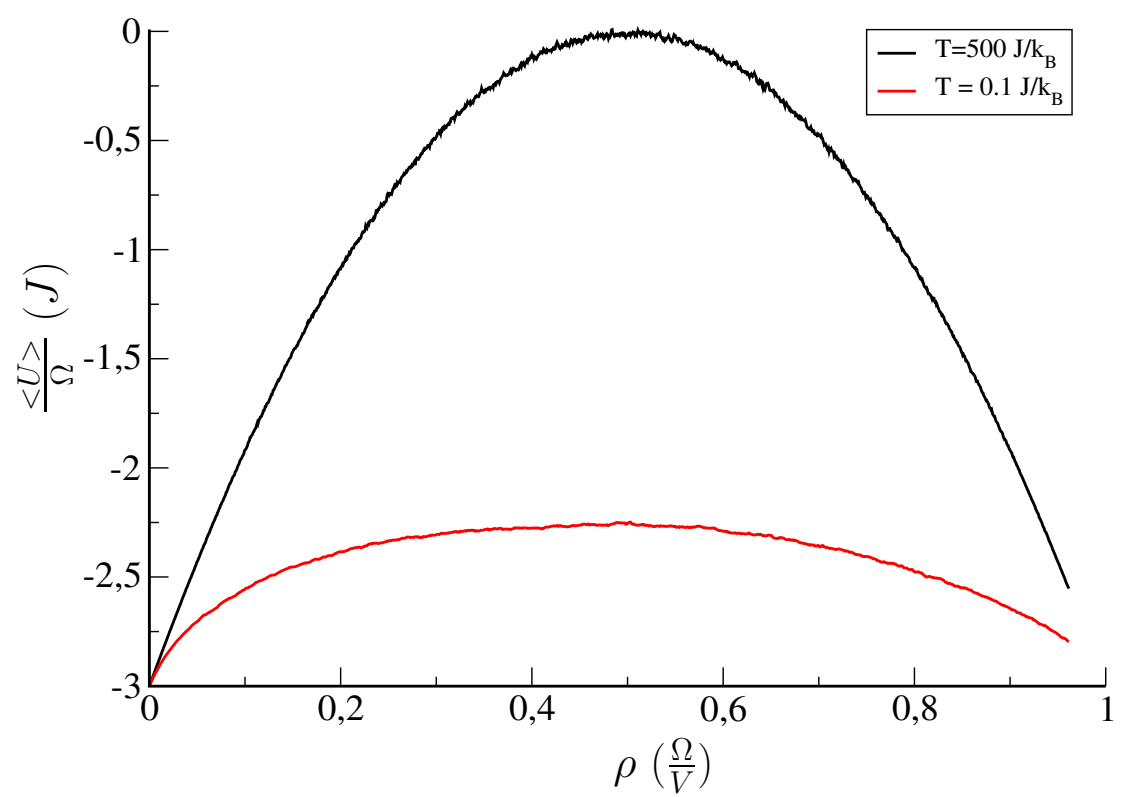

Figura 3.14: Estudo do modelo de Ising em três dimensões. No gráfico temse a energia interna média por partícula de líquido $U / \Omega(J)$ em função da variação da densidade de partículas de líquido $\rho\left(\frac{\Omega}{V}\right)$.

tura muitas vezes. Com isso é possível calcular a probabilidade de encontrar a placa na $2 h$ e na altura $2 h+1$.

Utilizando essas probabilidades, pode-se encontrar a diferença de energia livre $\mathcal{F}$ (Eq. 2.29) da ponte líquida e da força aplicada nas placas, calculados utilizando o método "Overlapping Distribution", para diferentes temperaturas. Para efeito de comparação, a energia e a força também foram calculadas utilizando a Eq.2.17 para calcular a energia do agregado e a Eq.3.1 para calcular a força da ponte líquida na placa;

Nestas simulações foram utilizados 10000 partículas de líquido e a dimensão da rede foi de $60 \times 60 \times 52$. Cada configuração de energia corresponde à tentativa de trocar todas as 10000 partículas de líquido por partículas de gás. Para calcular a energia livre realizou-se 3000000 de sorteios entre duas al- 


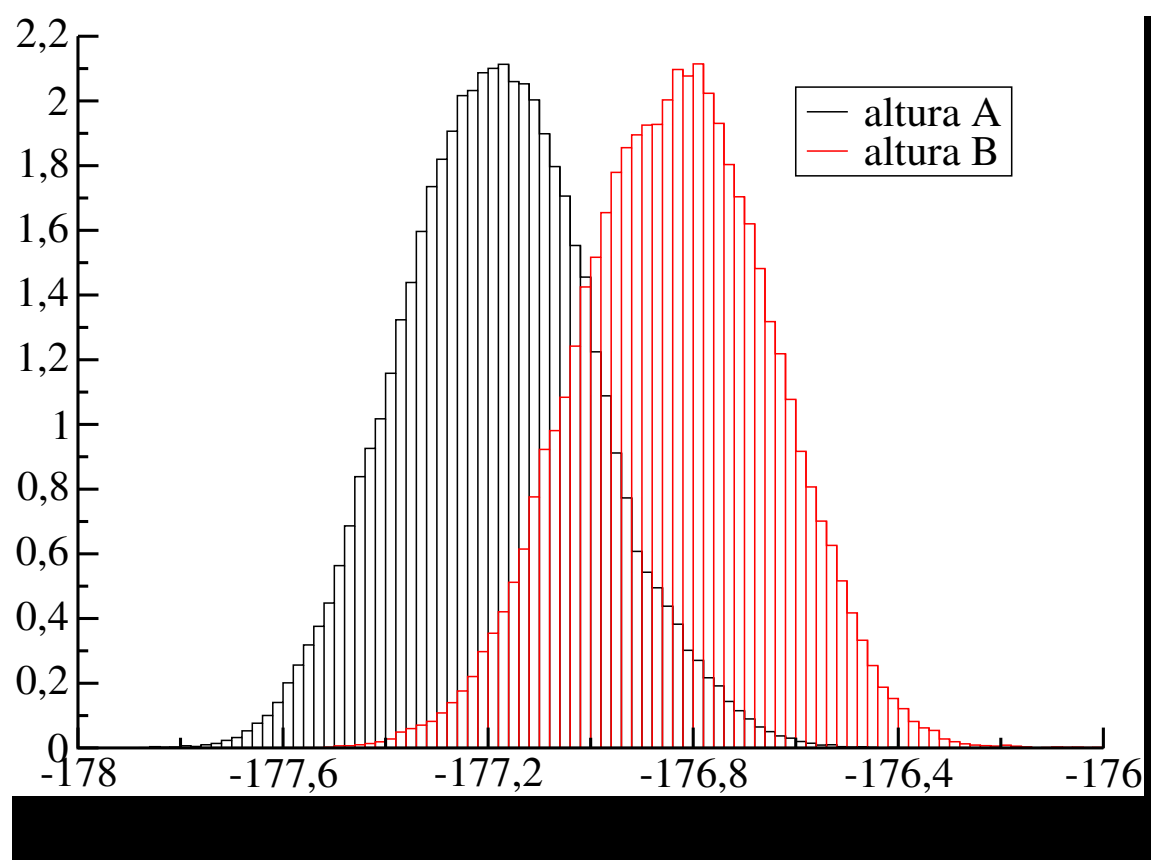

Figura 3.15: Comparação entre a distribuição de energia normalizada entre duas alturas: $h=26$ (preto) e $h=27$ (vermelho) para a temperatura $T=$ 1.25. Estes histogramas foram construídos com 198000 configurações

turas consecutivas. As temperaturas $T$ utilizadas foram: $0,7 \mathrm{~J} / k_{\mathrm{B}}, 0,9 \mathrm{~J} / k_{\mathrm{B}}$, $0,93 \mathrm{~J} / k_{\mathrm{B}}, 1,25 \mathrm{~J} / k_{\mathrm{B}}, 1,40 \mathrm{~J} / k_{\mathrm{B}}$ e $1,50 \mathrm{~J} / k_{\mathrm{B}}$ e o número de configurações utilizadas, respectivamente, para cada uma destas temperaturas foi: 198000, 160000, 63435, 198000, 209000 e 248000.

Na Figura 3.16 está apresentado a energia média para cada altura e temperatura $T$ utilizando a Eq. 2.17 e as barras de erro representam o desvio padrão. Esse desvio padrão também pode ser observado nos histogramas apresentados na Fig. 3.15. A Fig. 3.17 mostra a energia livre calculada utilizando o método de "Overlaping Distribution" e a Fig. 3.18 mostra a comparação entre os dois métodos.

De forma análoga a força exercida pelo líquido nas placas planas foi cal- 


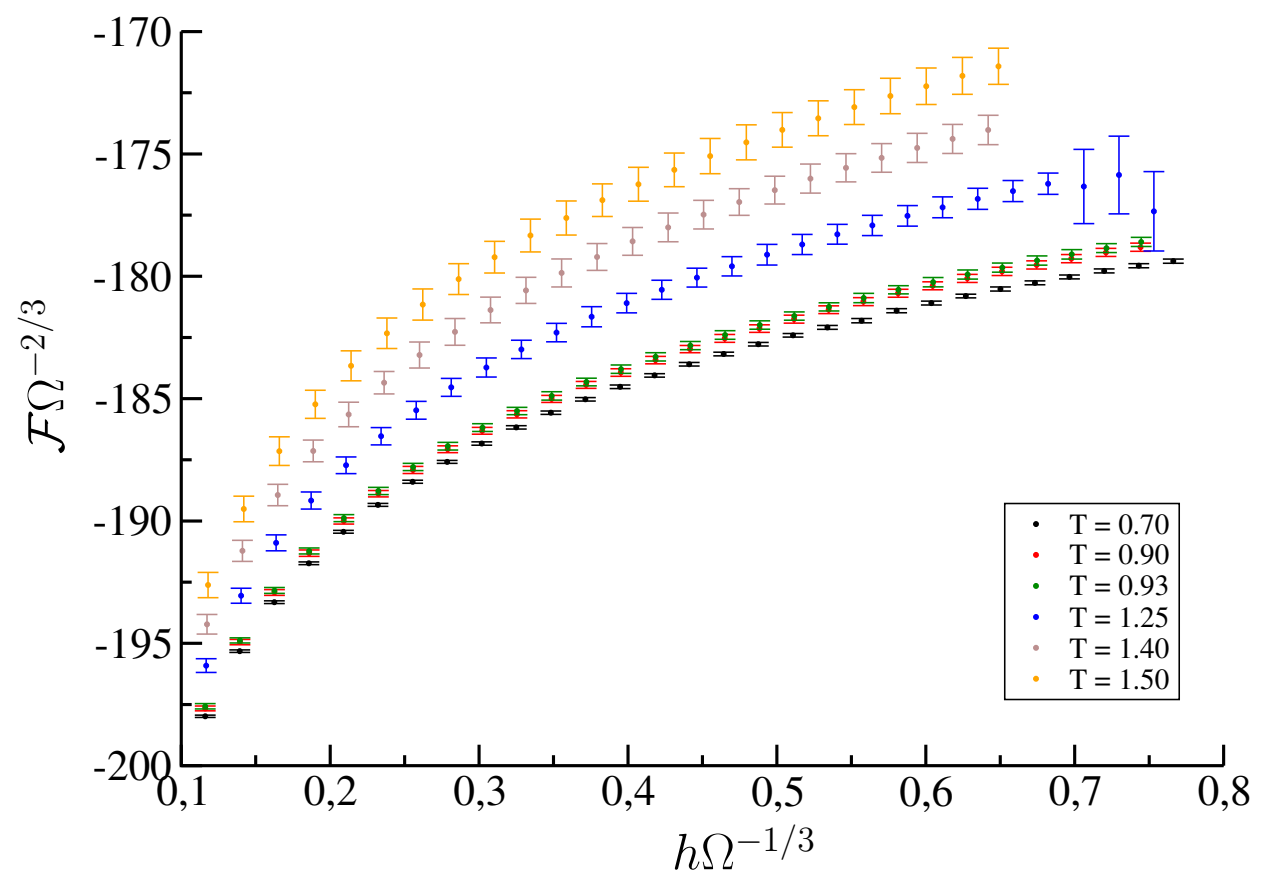

Figura 3.16: Gráfico da energia interna da ponte líquida normalizada pelo volume da ponte líquida em função da meia altura entre as placas normalizada pelo volume. Para cada uma das temperaturas $0,7 \mathrm{~J} / k_{\mathrm{B}}, 0,9 \mathrm{~J} / k_{\mathrm{B}}$, $0,93 \mathrm{~J} / k_{\mathrm{B}}, 1,25 \mathrm{~J} / k_{\mathrm{B}}, 1,40 \mathrm{~J} / k_{\mathrm{B}}$ e $1,50 \mathrm{~J} / k_{\mathrm{B}}$, foram utilizados os seguintes números de configurações: 198000, 160000, 63435, 198000, 209000 e 248000. As barras de erro representam o desvio padrão para cada altura.

culada utilizando os dois métodos, energia interna Fig. 3.19, e "Overlaping Distribution" Fig. 3.20. Os dois métodos foram comparados na Fig. 3.21. 


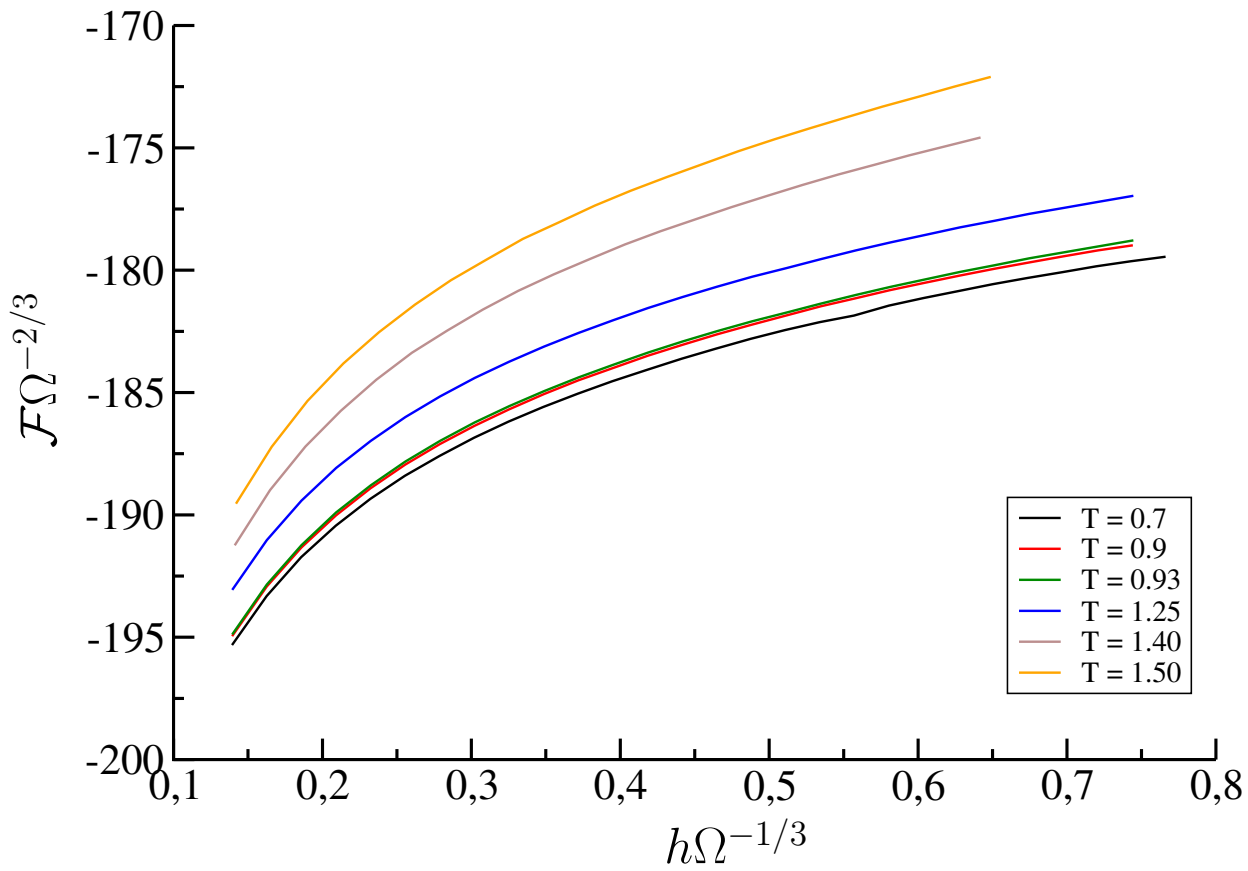

Figura 3.17: Gráfico da energia livre normalizada pelo volume $\mathcal{F} / \Omega^{-2 / 3}$ em função da altura normalizada pelo volume $h / \Omega^{-1 / 3}$. A diferença de energia livre foi calculada utilizando o método "Overlaping Distribution". Como este método calcula a diferença de energia livre entre dois estados, para calcular a energia livre de cada altura, as diferenças de energia livre foram somadas. 


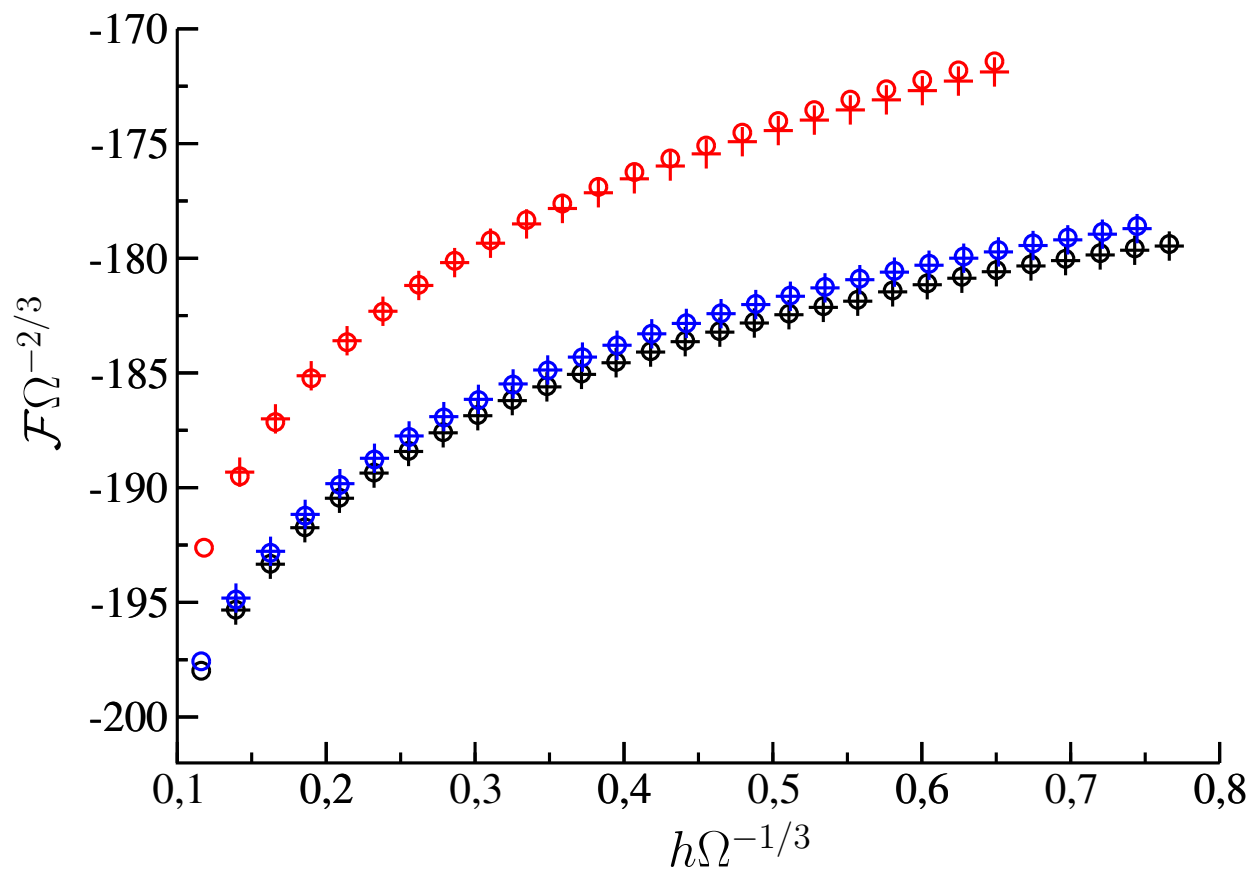

Figura 3.18: Comparação da energia livre utilizando o método de "Overlapping Distribution" representado pelo símbolo +, e a energia interna do sistema representado por um círculo, para três temperaturas diferentes: $T=0,70 \mathrm{~J} / k_{\mathrm{B}}, T=0,93 \mathrm{~J} / k_{\mathrm{B}}$ e $T=1,50 \mathrm{~J} / k_{\mathrm{B}}$, respectivamente nas cores preto, azul e vermelho. 


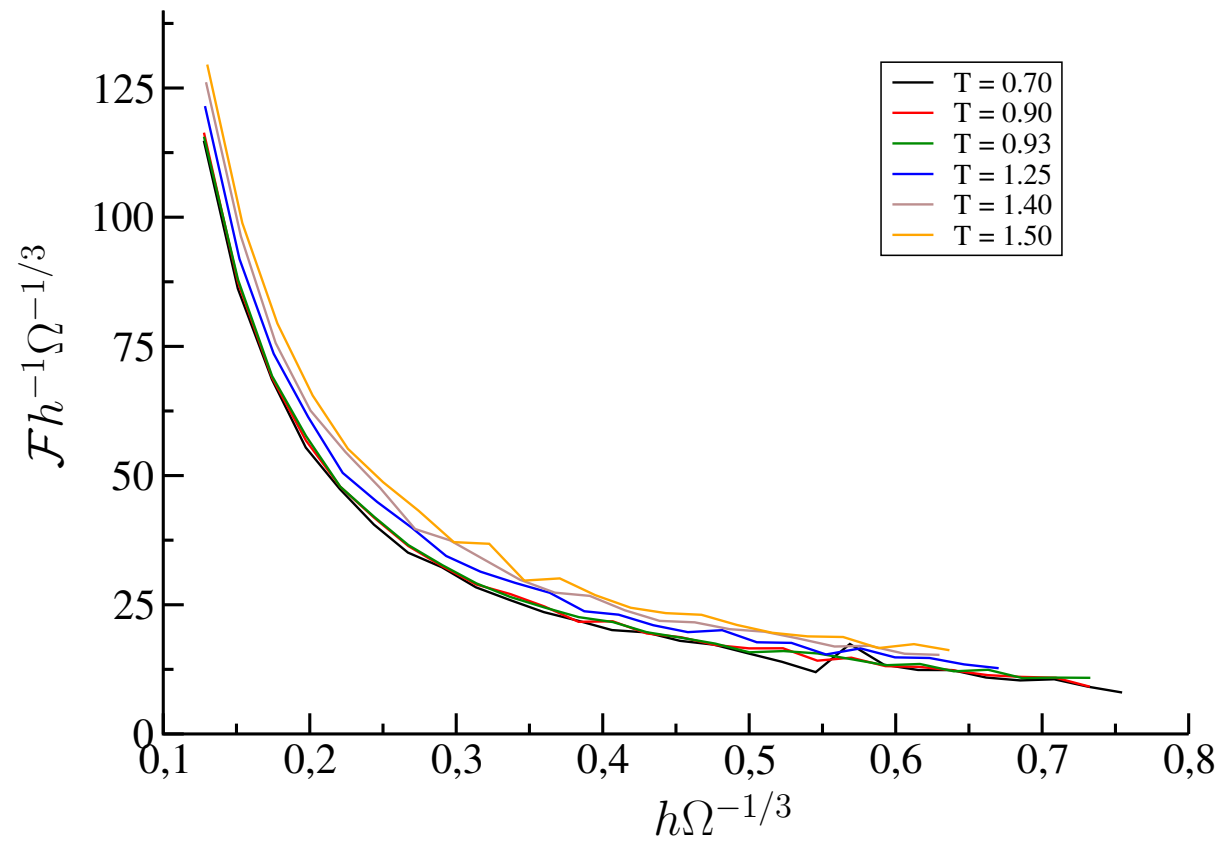

Figura 3.19: Gráfico da força normalizada pelo volume nas placas planas em função da altura normalizada pelo volume para as temperaturas $0,7 \mathrm{~J} / k_{\mathrm{B}}$, $0,9 J / k_{\mathrm{B}}, \quad 0,93 \mathrm{~J} / k_{\mathrm{B}}, 1,25 \mathrm{~J} / k_{\mathrm{B}}, 1,40 \mathrm{~J} / k_{\mathrm{B}}$ e $1,50 \mathrm{~J} / k_{\mathrm{B}}$. Neste caso foi utilizado a diferença de energia interna para calcular a força. 


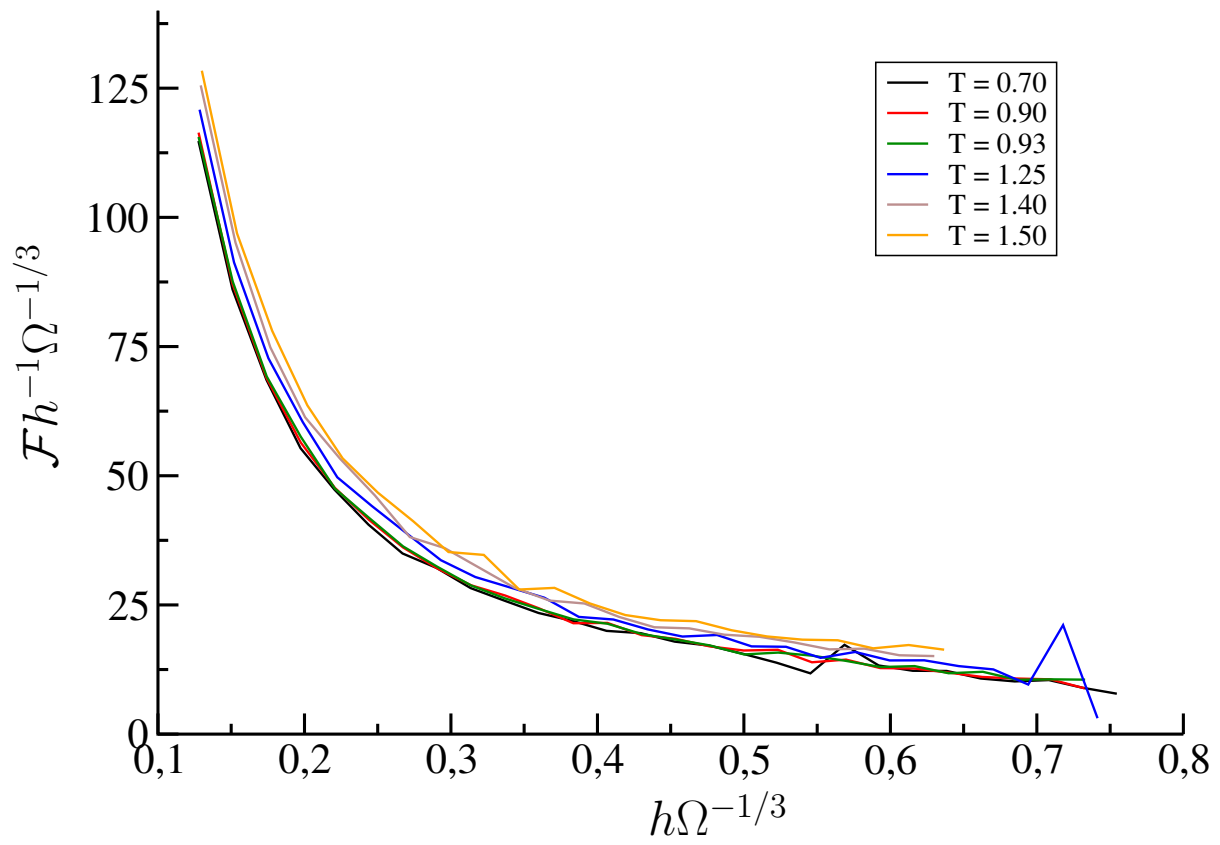

Figura 3.20: Gráfico da força na placa para cada altura e temperatura utilizando o método "Overlapping Distribution". 


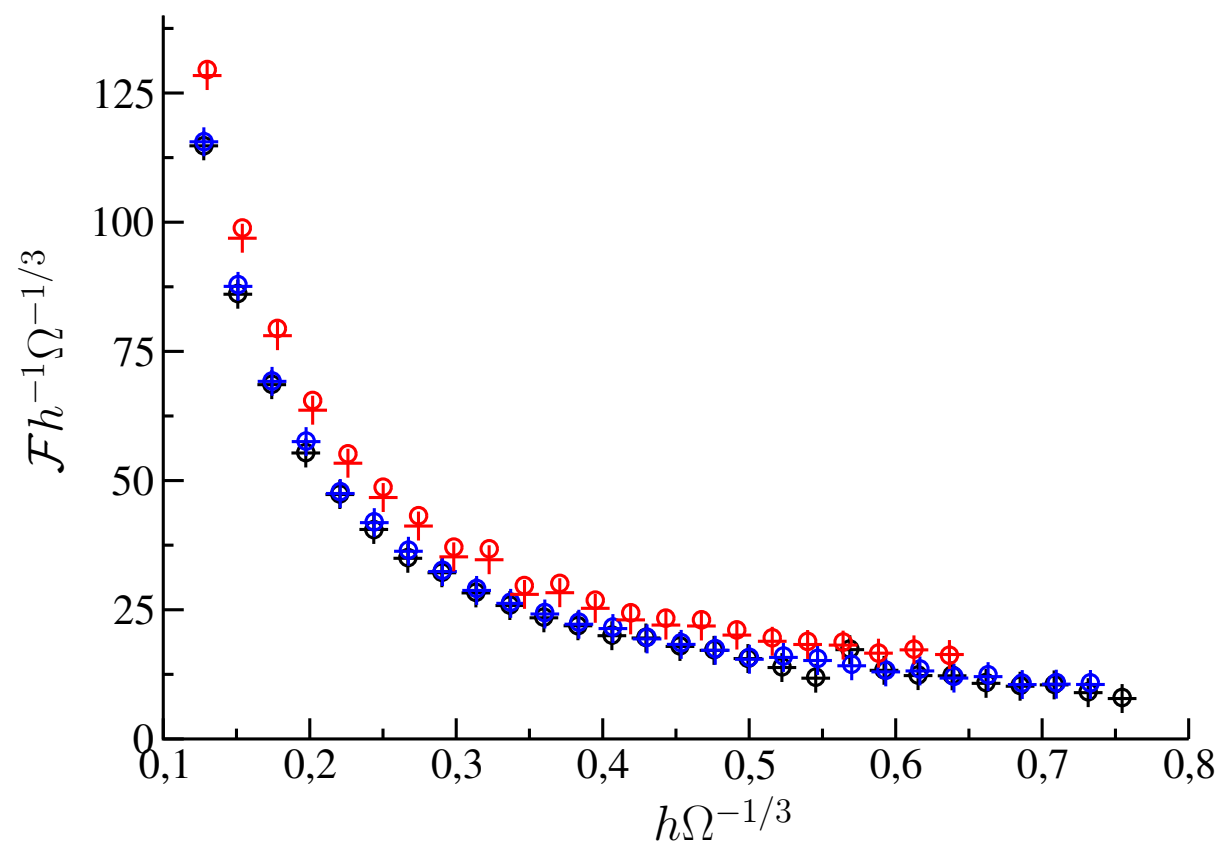

Figura 3.21: Comparação da força utilizando o método de "Overlapping Distribution", +, e a energia interna do sistema, círculos, para três temperaturas diferentes: $T=0,70 \mathrm{~J} / k_{\mathrm{B}}, T=0,93 \mathrm{~J} / k_{\mathrm{B}}$ e $T=1,50 \mathrm{~J} / k_{\mathrm{B}}$, respectivamente nas cores preto, azul e vermelho. 


\section{Capítulo 4}

\section{Conclusão}

O modelo para pontes líquidas na escala macroscópica mostrou a diferença de energia livre existente entre a ponte líquida e uma gota formada na placa plana para diferentes ângulos de contato $\Theta$, como pode ser verificado na Fig. 3.1. Os resultados numéricos ficaram em acordo com a solução analítica o que válida o modelo possibilitando sua aplicação em geometrias mais complexas. A validação do modelo também ocorreu a nível termodinâmico. O estudo do sistema simplificado de duas partículas de líquido mostrou que a metodologia utilizada para estudar a energia interna e o calor específico do modelo numérico corresponde ao obtido analiticamente.

Pode-se hipotetizar que a perda de energia causa instabilidade mecânica e pode gerar o ruído de crepitação. Pode-se notar da Fig. 3.1 que $\Delta \mathcal{F}$ aumenta à medida que $\Theta$ diminui. A alteração em $\Theta$ pode corresponder a uma alteração nas propriedades do fluido que recobrem as vias aéreas. Consequentemente, a diferença de energia livre $\Delta \mathcal{F}$ pode corresponder a uma mudança na intensidade no ruído de crepitação emitido. A conexão entre as 
propriedades das superfícies dos fluídos no pulmão e o ruído de crepitação gerado durante a ruptura da ponte líquida ou sua formação é crucial para o entendimento de patologias de muitas doenças respiratórias e para aprimorar o diagnostico clínico. Entretanto, esse modelo para pontes líquidas macroscópicas no presente momento não pode ser aplicado para a fisiologia pulmonar principalmente devido às diferenças topológicas entre nosso estudo e o caso real das pontes líquidas nas vias aéreas. Pode-se notar que a ponte líquida na via aérea formará não somente devido à contração geométrica da via aérea, mas também devido à flutuação no fluxo de ar. Além disso, uma mistura complexa de fluidos orgânicos recobrindo a via aérea não pode ser tratado como um fluido Newtoniano. Portanto, uma comparação direta entre a energia perdida durante a ruptura da ponte líquida na via aérea e o sistema que está sendo trabalhado aqui não é clara ainda.

Com relação ao efeito de escala, conseguiu-se interpretar parâmetros que estão relacionados a relação área volume do sistema além da distância efetiva em que a ponte se forma. Esses parâmetros podem ser utilizados para mapear as dimensões utilizadas no modelo em dimensões físicas reais.

Com relação ao cálculo da energia livre, não foi encontrado nenhuma dependência da entropia na energia livre utilizando a metodologia "Overlaping Distribution". Entretanto, existe uma discussão sobre a precisão deste método. O método de "Umbrela" pode corrigir este problema e pode dar uma melhor estimativa sobre a energia livre do sistema.

Com relação ao potencial de interação, como discutido no corpo do texto, foi utilizado interação de primeiro, segundo e terceiros vizinhos. Esse potencial foi ajustado de forma a imitar um potencial de longo alcance servindo 
também de ajuste para a obtenção de um ângulo de contato $\Theta$ desejado. A ideia do modelo é de que cada sítio da rede seja interpretada como uma parcela microscópica do sistema, ou seja uma gotícula. Nesse caso essa interação poderia ser vista como um potencial de Hamaker [57, 58] que é normalmente utilizado para estudar a estabilidade de emulsões. 


\section{Apêndice A}

\section{A Equação de Kelvin}

A equação de Kelvin relaciona a pressão de vapor sobre a superfície de um fluido de forma esférica $P_{S}$ e de área $A$ com a pressão normal de vapor $P_{0}$ desse fluido em uma superfície plana de mesma área $A$ [36]. A pressão normal de vapor é a pressão exercida pelo vapor em equilíbrio termodinâmico com sua fase condensada. Para deduzir a equação de Kelvin deve-se considerar um gás ideal, cuja equação de estado pode ser escrita como:

$$
P V=n R T
$$

em que $P$ é a pressão, $V$ é o volume, $n$ é o número de mols, $R$ é a constante universal dos gases e $T$ é a temperatura.

Então, deve-se calcular a variação da energia livre de Gibbs $\Delta G$ do gás ideal. A energia livre de Gibbs é dada por:

$$
G=U-T S+P V
$$


onde $U$ é a energia interna e Sé a entropia. Considerando a temperatura e o número de mols constante, a variação da energia livre de Gibbs do gás ideal é dado por:

$$
\Delta G=\int_{P_{0}}^{P_{S}} P d V
$$

em que $P_{0}$ é a pressão normal de vapor e $P_{S}$ é a pressão sobre toda a superfície da gota. Utilizando a equação (A.1) obtem-se:

$$
\Delta G=n R T \ln \frac{P_{S}}{P_{0}}
$$

Agora, é necessário calcular a variação da energia livre de Gibbs $\Delta G$ para a gota. Pode-se escrever que a variação da energia livre, assumindo que o volume da gota permanece constante, é dado por:

$$
\Delta G=\int V d P=V \Delta P
$$

Utilizando a equação (2.2):

$$
\Delta G=\frac{2 \gamma V}{r}
$$

Assim, igualando as equações (A.4) e (A.6), obtem-se a equação de Kelvin que relaciona a pressão normal de vapor com a pressão em uma superfície de uma esfera:

$$
\ln \frac{P_{S}}{P_{0}}=\frac{\gamma v}{R T} \frac{2}{r}
$$





\title{
Apêndice B
}

\section{Artigo Submetido}

Crackling Sound Generation During the Formation of Liquid Bridges: A Lattice Gas Model

Alexandre B. de Almeida

Instituto de Fisica, Universidade de São Paulo, SP, Brazil

Sergey V. Buldyrev

Department of Physics, Yeshiva University, New York, NY, 10033 USA

Adriano M. Alencar

Instituto de Fisica, Universidade de São Paulo, SP, Brazil

\begin{abstract}
Due to abnormal mechanical instabilities, liquid bridges may form in the small airways blocking airflow. Liquid bridge ruptures during inhalation are the major cause of the crackling adventitious lung sound, which can be heard using a simple stethoscope. Recently, Vyshedskiy and collegeus [1] described and characterized a crackle sound originated during expiration. However, the mechanism and origin of the expiratory crackle are still controversial. Thus, in this paper, we propose a mechanism for expiratory crackles. We hypothesize that the expiratory crackle sound is a result of the energy released in the form of acoustic waves during the formation of the liquid bridge. The magnitude of the energy released is proportional to the difference in free energy prior and after the bridge formation. We use a lattice gas model to describe the liquid bridge formation between two parallel planes. Specifically, we determine the surface free energy and the conditions of the liquid bridge formation between two parallel planes separated by a distance $2 h$ by a liquid droplet of volume $\Omega$ and contact angle $\Theta$, using both Monte Carlo simulation of a lattice gas model and variational calculus based on minimization of the surface area with the volume and the contact angle constrained. We numerically and analytically determine the phase diagram of the system as a function of the dimensionless parameter $h \Omega^{-1 / 3}$ and $\Theta$. We can distinguish two different phases: one droplet and one liquid bridge. We can observe a hysteresis curve for the energy changes between these two states, and ob-
\end{abstract}


served a finite size effect in the bridge formation. We compute the release of free energy during formation of the liquid bridge, and we discuss the results in terms of system size. We also calculate the force exerted from liquid bridge on the planes by studying the dependence of the free energy on the separation between the planes $2 h$. The simulation results are in agreement with the analytical solution.

Keywords: crackle, pots, instabilities, sound PACS: 04.60.Nc, 04.20.Fy, 89.20.-a

\section{Introduction}

The lung airways are coated with surfactants and other organic fluids, and the surface force generated at the air-liquid interface plays a major role in breathing mechanics $[2,3,4]$. In certain lung diseases, the surface properties of these fluids are changed and liquid bridges may form in small airways, closing them, and consequently impairing gas exchange $[5,6,7,8,9]$. The stability, the rupture, and formation conditions of the liquid bridge are strongly influenced by surface tensions at the air-liquid interface and the nature of the solid-liquid contact.

During inspiration, liquid bridges that are closing the airways may pop open due to mechanical instabilities $[10,11,12]$ and emit a discrete sound event called pulmonary crackle, which can easily be heard using a stethoscope. Lung crackle is an important adventitious lung sound for diagnosis of lung diseases $[13,14,15,16,17,18]$ and has been an interesting problem in physics, since they are caused by formation and rapture of capillary liquid bridges $[19,20,21,22]$ which have been studied extensively in other branches of physics. Recently, Vyshedskiy and collegues [1] systematically examined the relationship between inspiratory and the uncommon expiratory crackles. These observations were quantitatively consistent with stress relaxation quadrupole hypothesis of crackle generation [23], in which crackles are generated either by the opening or closing of the airways. Here, we evaluate a hypothesis that the expiratory crackles are generated by liquid bridge formation during the closing of the airway. Analogously, the inspiratory crackles are produced by the liquid bridge raptures, a problem studied numerically and analytically in Ref. [22]. To study this problem, we modeled the liquid bridge in a simple geometry: the liquid is trapped between two plane surfaces [22]. 


\subsection{Lattice Gas Model for Liquid Bridge}

Lattice gas models have been studied to analyze, interpret and predict experimental results including such diverse phenomena as atomic force microscopy [24, 25], sintering [26, 27], cellular motors [28], and lung sounds [22]. We use a simplified 3D lattice gas model that has been developed to explain inspiratory crackle generation during liquid bridge rupture [22] and validate it by an exactly solvable analytical model based on variational principles. In both models, numerical and analytical, the liquid bridges are axially symmetrical between two semi-wet planes, where the base radius $R$ at both planes is not fixed. For this particular geometry, we could distinguish two stable configurations: (i) when the liquid phase forms a bridge between the two planes, we call it a closed state, and (ii) when the liquid phase form a droplet on the plane, we call it an open state [22].

That lattice gas model includes particles of three types: liquid $(l)$, solid $(s)$, and gas $(g)$. A system consists of liquid of total volume $\Omega$, two solid planes separated by distance $2 h$, and surrounding gas. The lattice size is $60 \times 60 \times 52$ with 10000 liquid particles. We simulate the formation of the liquid bridge between the planes and its rupture using the Monte Carlo method with the Metropolis algorithm. There is no exchange between identical particles, and the exchange between gas and liquid particles are performed randomly according to the Kawasaki dynamics and weighted by the Boltzmann factor, while the solid particles are fixed to maintain the geometry of the planes. We can move the planes in $z$ direction to change their separation $2 h$. Each particle has 26 neighbors: $6+12+8$, with distances $1, \sqrt{2}$ and $\sqrt{3}$, respectively. To model the liquid-liquid interaction and liquid-solid interaction, we reduce the interaction strength at distances $\sqrt{2}$ and $\sqrt{3}$ with respect to the interaction strength between the nearest neighbors [22]. The contact angle $\Theta$ is set by adjusting the solid-liquid interaction. During this process the lattice gas model evaluates several physical properties such as energy, liquid bridge shape, and surface area.

\subsection{Analytical Solution}

We use the analytical solution to validate the lattice gas model of liquid bridge between two parallel planes [22]. All relevant characteristics of the liquid bridge between two parallel planes is found in terms of elliptic integrals, which depend on their contact angle $\Theta$ and the bridge neck to base ratio $a=A / R$, where $A$ is the radius of the neck of the bridge and $R$ is the radius of the circle of contact between the bridge and the plane [22]. 
A single droplet of liquid attached to one of the two planar surfaces will acquire a shape of a spherical segment of radius $R_{0}$, with the center at $\left(x=0, y=0, z= \pm\left[h+R_{0} \cos \Theta\right]\right)$ forming a circular intersection with the plane of the radius $\rho_{0}=R_{0} \sin \Theta$, where

$$
R_{0}=\left(\frac{6 \Omega}{2 \pi\left(2-3 \cos \Theta+\cos ^{3} \Theta\right)}\right)^{1 / 3}
$$

In this case, the minimal separation of the planes $h_{\min }$ before the formation of the liquid bridge is

$$
2 h_{\min }=R_{0}(1-\cos \Theta) .
$$

It was shown in Ref.[22] that the liquid bridge of the same volume $\Omega$ is stable at this separation and its free energy $\mathcal{F}_{b}\left(\Omega, h_{\min }\right)$ is smaller than the free energy of the single droplet $\mathcal{F}_{0,1}(\Omega)$, which is given by

$$
\mathcal{F}_{0,1}=\pi \gamma R_{0}^{2}\left[2(1-\cos \Theta)-\sin ^{2} \Theta \cos \Theta\right],
$$

and

$$
\mathcal{F}_{b}(a) \equiv 2 \pi h_{\min }^{2} \frac{2 \sigma(a, 1)-\cos \Theta}{u(a, 1)^{2}} .
$$

where $\gamma$ is the surface tension, and $a$ is a dimensionless bridge neck radius, which can be determined from the equation,

$$
\Omega=2 \pi h_{\min }^{3} \frac{v(a, 1)}{u(a, 1)^{3}},
$$

and $u(a, 1), v(a, 1)$, and $\sigma(a, 1)$ can be expressed in terms of Legendre elliptic integrals $F(\phi, k)$ and $E(\phi, k)$ as

$$
\begin{aligned}
u(a, r)= & a \tilde{F}\left(k, \phi_{r}\right)+b \tilde{E}\left(k, \phi_{r}\right) \\
\sigma(a, r)= & (b+a) b \tilde{E}\left(k, \phi_{r}\right) \\
v(a, r)= & {\left[-\mathcal{R}(a, r)-a^{2} b \tilde{F}\left(k, \phi_{r}\right)\right.} \\
& \left.+b\left[3 a b+2\left(a^{2}+b^{2}\right)\right] \tilde{E}\left(k, \phi_{r}\right)\right] / 3,
\end{aligned}
$$


where

$$
\begin{array}{ll}
b= & \left(\frac{1-a \sin \Theta}{\sin \Theta-a}\right) \\
k= & s \sqrt{b^{2}-a^{2}} / b, \\
\mathcal{R}(a, r)= & r \sqrt{\left(r^{2}-a^{2}\right)\left(b^{2}-r^{2}\right)}, \\
\phi_{r}= & \arcsin \sqrt{\left(b^{2}-r^{2}\right) /\left(b^{2}-a^{2}\right)} \\
\tilde{F}(k, \phi)= & F(k, \pi / 2)-F(k, \phi) \\
\tilde{E}(k, \phi)= & E(k, \pi / 2)-E(k, \phi) .
\end{array}
$$

This suggests that as soon as a droplet touches the opposite plane, it forms a symmetrical bridge. As soon as the bridge forms, it starts to attract the opposite planes with the force

$$
F=2 \pi \gamma \frac{h(1-a \sin \Theta)}{1\left(1-a^{2}\right) u(a, 1)},
$$

which is a monotonically decreasing function of $h$. Thus formation of the bridge may lead to the collapse of the space between the planes if the external force keeping the planes apart is less than $F$. The existing bridge loses its stability and breaks if the half distance between the planes becomes greater than

$$
h_{\max }=u\left(a_{c}, 1\right)\left(\frac{\Omega}{2 \pi v\left(a_{c}, 1\right)}\right)^{\frac{1}{3}},
$$

where $a_{c}$ is the critical neck-base ratio $a_{c}=\tan (\Theta / 2)$, for $\Theta>31^{\circ}$.

We study the dependence of the energy gap during the liquid bridge formation with contact angle $\Theta$, and relate this energy gap to the crackling bursting sound in the lung. We can compute the free energy difference $\Delta \mathcal{F}=$ $\mathcal{F}_{b}\left(\Omega, h_{\min }\right)-\mathcal{F}_{0,1}(\Omega)$ between the energy of the droplet and the bridge, which will give us the energy released during the liquid bridge formation, see Fig.1.

\section{Results and Discussion}

In order to compare the results of the lattice gas model with the analytical calculations we introduce a critical distance $2 h_{c}$ between the planes at which a single droplet of total volume $\Omega$ forms a bridge. The abrupt decrease in free energy will then disturb the system as a mechanical perturbation, generating a short bursting sound that we associate with an expiratory crackle [1]. We show that in the lattice gas model $h_{c}$ is uniquely determined by the dimensionless parameter $\eta(\Theta)=h_{c} \Omega^{-1 / 3}$ which depends only on the contact angle, see Fig. 2. 


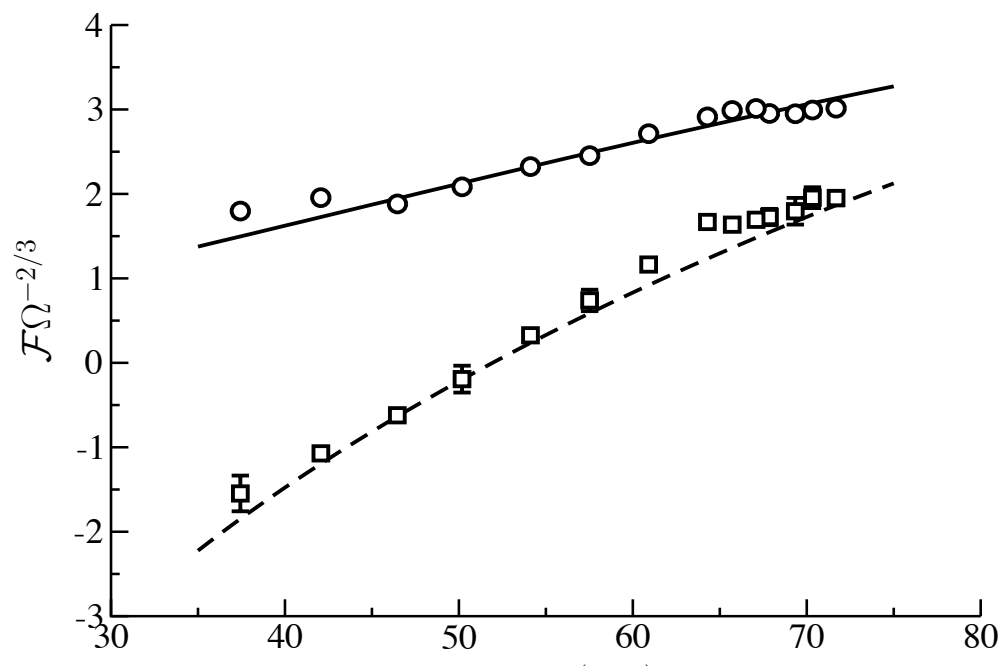

$\Theta(\operatorname{deg})$

Figure 1: The normalized free energy $\mathcal{F} \Omega^{-2 / 3}$ as a function of the contact angle $\Theta$ at the moment of the liquid bridge formation with the distance between the planes $2 h_{\min }$. The analytical and corresponding numerical maximal free energy of the droplet (continuous line Eq. (3)/open circles), and the liquid bridge (dashed lines Eq. (4)/open squares). The lattice size is $60 \times 60 \times 52$ with 10000 liquid particles. 


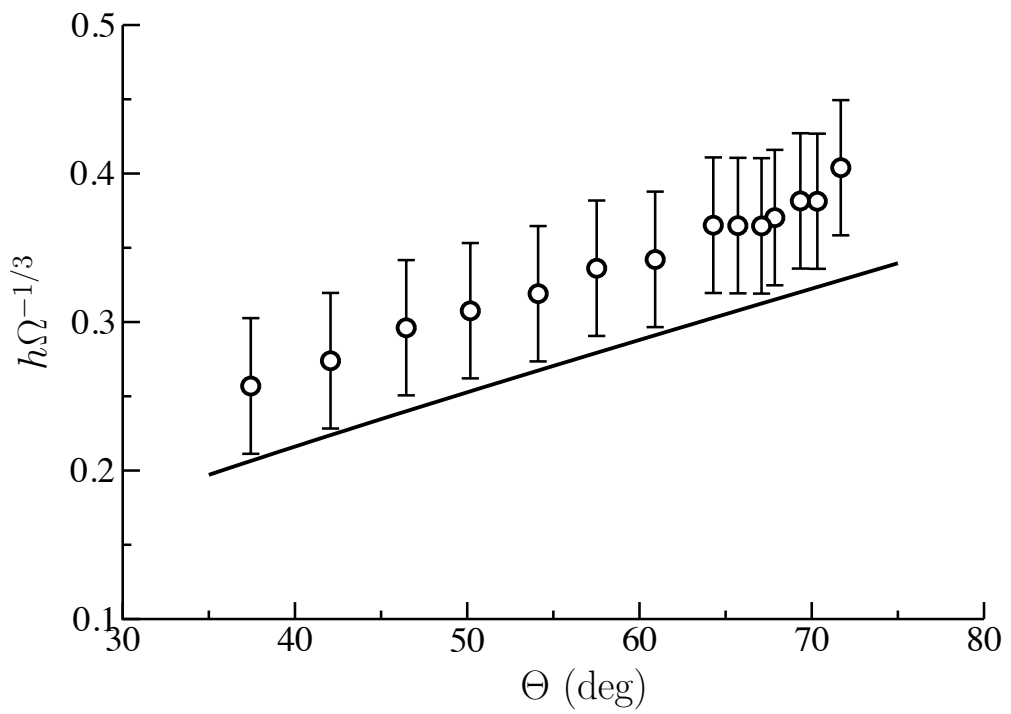

Figure 2: The minimal half-distance between the two planes $h_{\min }$, for which the droplet is stable, computed using Eq.(2) (solid line) [22]. Circles indicate the value of $h_{c}$ for which the bridge forms during the numerical simulation. The vertical error bars correspond to 1 lattice cell resolution for the distance in which the rupture is observed. The lattice size is $60 \times 60 \times 52$ with 10000 liquid particle. 
Indeed, we found similarities between simulation and analytical results (Fig. 2). However, Fig. 2, shows that the bridge always forms before the minimal $h_{\text {min }}$ position predicted by analytical calculation. In order to explain this, we notice that due to the effect of the temperature, there are aways particles of liquid at the surface of both planes and in the bulk, forming the vapor. In a system with a single droplet in one of the planes moving down, there will be a moment in which the droplet gets very close to the second plane, let's say, three sites apart, see Fig. 3. The surface of the droplet has a spontaneous deformation due to exchange particles at the liquid-gas interface, associated with the surface's free energy change in the amount of a few $k_{\mathrm{B}} T$. Furthermore, the vapor in the gap between the droplet and the opposite plane will generate an instability, attracting more vapor to the gap, starting an avalanche which will lead to the liquid bridge formation, illustrated in Fig. 3. The vapor effect is very strong and most of the bridges will be formed at this point, which explains the difference in results from the analytical calculation and the lattice gas model. We must highlight that the vapor effect we describe here is not numerical diffusion. In our simulation the exchange dynamics is not limited to neighboring sites, it can happen between any two sites of the lattice. However, we tested the model only allowing particle exchanges within neighbors range and the vapor effect were still present. Indeed, there are always some particles attached to the plane and some particles protruding from the droplet by one site. Hence the particle inserted in between the droplet and the plane may attract to both the droplet and the plane and hence has a larger chance to stay there than in the open space far away from the liquid and plane. Successful insertion of such a particle may lead to an avalanche of insertions into the nearby sites which rapidly fills the gap between the droplet and the wall. Thus, the liquid bridge always forms before the droplet touches the plane. Because of this, we found a small difference between numerical and analytical solutions. The analytical model does not have this effect, because it completely neglects the vapor, and the liquid interface roughness. Thus, the vapor effect can not be predicted in the analytical calculation, but it can be observed in our model and experimentally in the atomic force microscopy [29].

We can notice that the contact angle $\Theta$ does not change much in different system sizes. Next we study the effect of the system size on the bridge formation, see Fig. 4. Thus, we fixed all the interaction parameters $s_{i, j}$ at the value that generates a contact angle of approximately $60^{\circ}$, and then vary the system size. As we increase the system sizes, we also increase the amount 
(a)

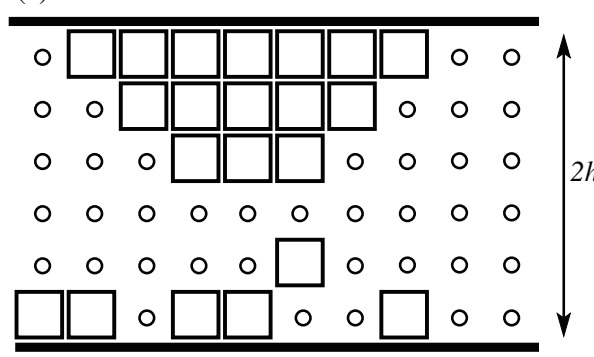

(b)

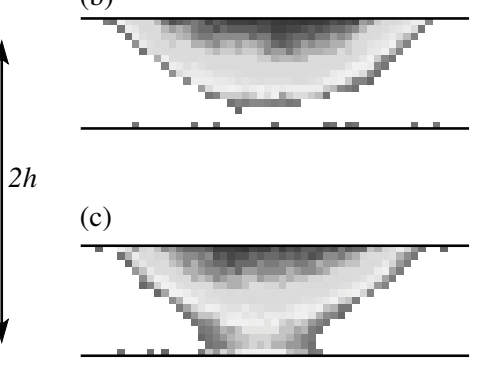

Figure 3: The illustration of the system near the liquid bridge formation, showing (a) Liquid particles (squares), air (circles) and the planes (thick lines). We call vapor the unconnected liquid particles. The liquid particles on the planes are wetting them, and all the remaining liquid particles form the droplet.

This illustration highlights the effect of the presence of vapor between the droplet and the wet bottom plane, which can lead to an early bridge formation. (b) The image of a typical case just before the liquid bridge is formed, where the top of the droplet is three lattice units from the wet bottom plane, and (c) just after the liquid bridge formation. 
of liquid particles, $\Omega^{\prime}$, always keeping constant the air/liquid fraction in the system. For each system size in the numerical model, we calculate the exact value of $\Theta$, and found the critical value where the liquid bridge is formed, numerically, $h_{c}$, and the analytical constraint $h_{\min }$. Due to the vapor effect, the liquid bridge will always form two or more sites before the droplet touches the second surface, independently of the system size. Thus, in Fig. 4 we plotted the numerical value of $h$ in which the bridge is formed, shifted by 3 sites $\left(2 h=2 h_{c}-3\right)$ and $h_{\min }$ against the system size. The plotted error bar represents the size of a lattice unit normalized by $\Omega^{1 / 3}$ and it decreases with system size. As the size of the system increases, the numerical value converges to the analytical expected value.

We can consider that the analytical and statistical liquid bridge model are composed of three kinds of elements: the planes, the liquid and the vapor. However, these objects have a different treatment in each model: in the analytical model they are continuous and are described by the surface tension in the interface between the elements; in the statistical model they are composed by particles and are described by the interaction of each particle with its neighbors. During the process of liquid bridge rupture [22], we found almost no finite size effects because the liquid bridge is formed and the whole system is almost continuous, so a single site makes a negligible effect, see Fig. 3c. However, during the process of the liquid bridge formation, we found a three-sites-effect which should only be neglected for very large systems. In other words, a single vapor particle between the droplet wall gap can make a difference, see Fig. 3b.

In Fig. 5 we plot the free energy in both situations, when we reduce the distance between the planes to form the liquid bridge (filled square) and when we separate (open circles) the planes until the liquid bridge breaks. We compare these numerical simulations with the analytical solution (red line) [22]. We can observe a hysteresis curve with two gaps in the free energy. The vertical red lines correspond to the height at the formation of the liquid bridge, $h_{\min }=0.25$, and at the rupture, $h_{\max }=0.68$ computed analytically using Eq. (2) and Eq (9), respectively. The two gaps in the free energy indicate a possible mechanism for crackling sound generation in the lung during the inspiratory and expiratory breathing [1, 23]. From both, analytical and numerical models we calculate the total free energy released during both, the formation and rupture of liquid bridges between two parallels planes. In both models, the formation has a greater loss of free energy than the rupture. In contrast with the experimental observations of Vyshed- 
skiy, in real human airways, showed the opposite results. However, in real experimental data, the generated crackle sound waves are transmitted from the generation site through the parenchyma until they reach the skin, when they are captured by an electronic stethoscope. During this process, several frequencies are attenuated as the chest and transducers work as coupled acoustic filters $[30,17,31,32]$. We hypothesize that the inspiratory crackle has lower frequencies and some of them are absorbed during the transmission, this hypothesis is supported by the experimental data [1]. Another difference is the topology. The study of Vyshedskiy and the stress relaxation quadrupole theory considers a cylindrical geometry while our model used two parallel planes.

Moreover, the continuous decrease in free energy as $h$ decreases will generate an attractive force between the two planes [33]. Our simulation is quasistatic, meaning that we move the upper plane by one step down and wait until the system reaches equilibrium. Since the plane remains in a fixed position at each step until moving to the next step, there is an external force $F_{e}$, which is equal to the resultant force due the surface tension of the liquid bridge. Applying thermodynamics, we relate the force to the partial derivative of the potential energy with respect to the distance:

$$
F_{e}=-\frac{1}{2}\left(\frac{\partial U}{\partial h}\right)_{T}+T\left(\frac{\partial F_{e}}{\partial T}\right)_{h} .
$$

We analyze the second term of the equation Eq. (10), which is related to the entropy of the system. We numerically estimate it by calculating the force at two different temperatures and constant height. We verify that this term is very small and can therefore be neglected. Thus, for a fixed distance $h$, and volume $\Omega$, we can write the external force, neglecting the entropic term, as

$$
f=-\frac{\partial U}{2 \partial h}
$$

In Fig. 6, we show $f$ computed from the liquid bridge energy and normalized half distance for three contact angles $\Theta=39^{\circ}, 50^{\circ}$, and $66^{\circ}$. The result we obtained is analogous to the analytical calculations obtained in the literature for a contact angle $\theta>30^{\circ}[33,22]$. We can notice that the force increases as $h$ decreases, which leads to the collapse of the space between the planes as observed in flexible tubes. We can interpret the calculated 


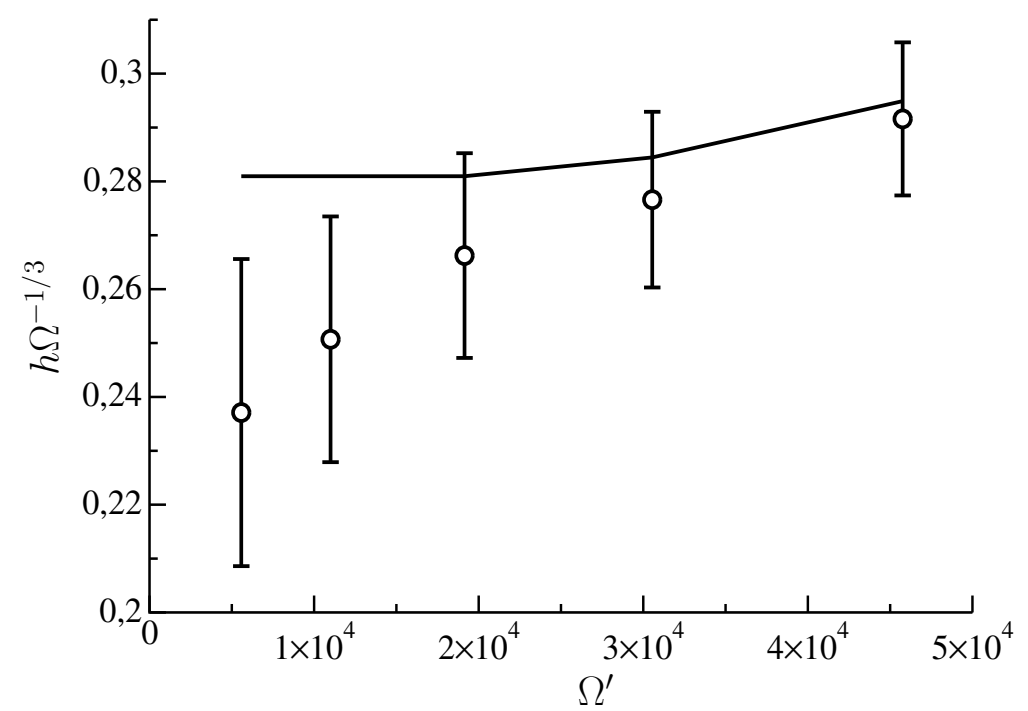

Figure 4: The system size analysis of the liquid bridge formation for $\Theta \approx 60^{\circ}$. We study five system sizes keeping the shape of a box and the ratio between liquid and gas particles at the same proportion. The edges of the cubic boxes are 40,50,60, 70 and 80 , and the corresponding total volume of liquid particles in the system $\Omega^{\prime}$ are $5574,11000,19140$, 30544, and 45760. The continuous thick line is the analytical liquid bridge formation distance $h_{\min }$

, normalized by $\Omega^{1 / 3}$, calculated based on the average volume of the droplet and average contact angle of 30 simulations. We can observe that the analytical calculation is not constant, due to the fact that, both the contact angle and the ratio $\Omega / \Omega^{\prime}$, change with system size. The open circles are the normalized half distance $h$ subtracted by three lattice sites for each system size. The error bars represent the normalized size of one lattice site above and bellow the average point. 


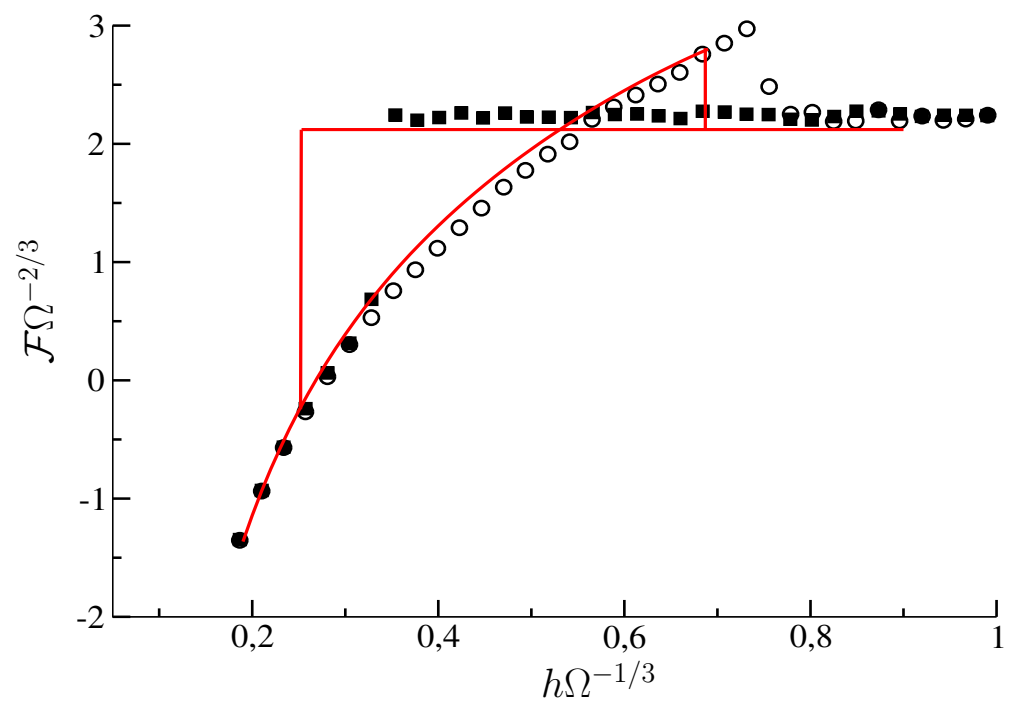

Figure 5: The normalized total free energy $\mathcal{F} \Omega^{-2 / 3}$ versus the normalized half distance $h \Omega^{-1 / 3}$ during liquid bridge formation (filled squares) and liquid bridge rupture (open circles). The vertical red lines represent the values of $h_{c}=0.687$ and $h_{\min }=0.247$ obtained analytically [22]. The continuous red lines are the analytical calculation of the normalized total free energy $\mathcal{F} \Omega^{-2 / 3}$ for the liquid bridge and for the droplet. Here we use $\Theta \approx 50^{\circ}, 10000$ particles of liquid and lattice size of $60 \times 60 \times 52$. The hysteresis in energy highlights the two regions of instability, when we stretch the bridge or compress the droplet. Each point in the curve is an average of ten simulations. 
force shown in Fig. 6 as the force needed to reopen a collapsed airway. This force is very high for small half distances $h$, and decreases as we increase $h$. Therefore, once the external force pulling the planes apart becomes larger the force attaching the planes to the bridge decreases and the bridge loses its stability.

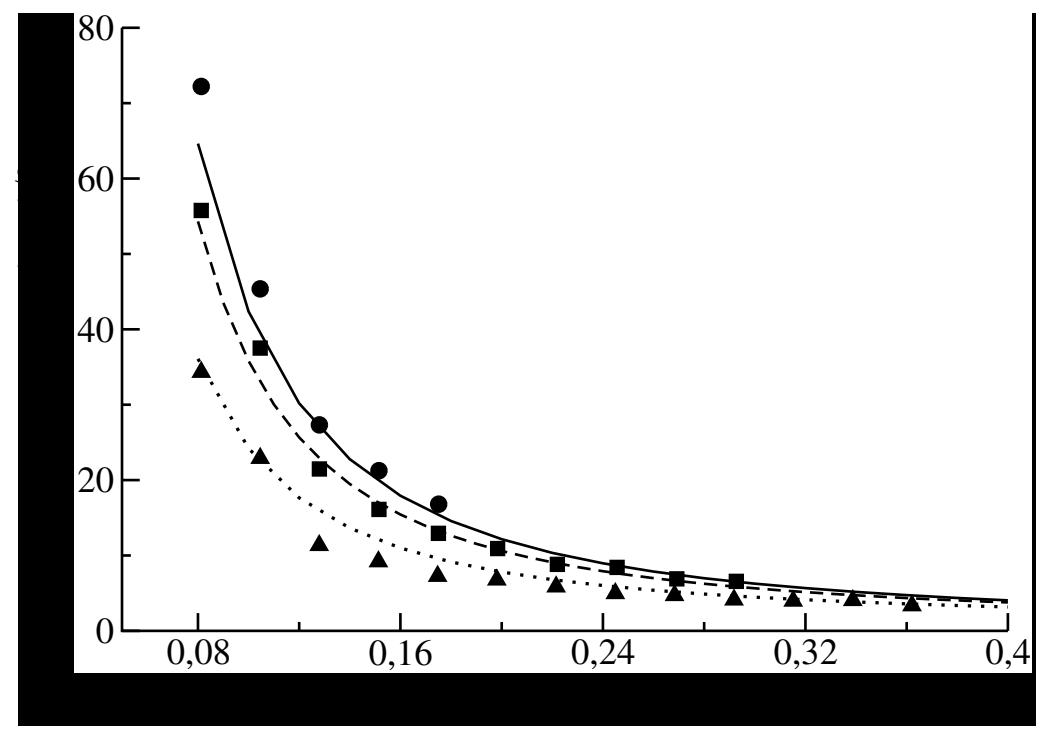

Figure 6: The force between the planes for three different contact angles $\Theta$ computed using Eq. (11). Circle, square and triangle represent $\Theta$ equal to $39^{\circ}, 50^{\circ}$, and $66^{\circ}$, respectively. The continuous, dashed and dotted lines are the analytical calculation force using Eq. (8) for $\Theta$ equal to $39^{\circ}, 50^{\circ}$, and $66^{\circ}$, respectively. We use a lattice size of $60 \times 60 \times 52$ and 10000 liquid particles.

\section{Conclusions}

From Fig. 1 we show the free energy difference between the two states, before and just after the liquid bridge formation, $\Delta \mathcal{F}$. The abrupt release of this energy causes mechanical instabilities and generates the bursting crackle sound. We can notice from Fig. 1 that $\Delta \mathcal{F}$ increases as $\Theta$ decreases, which 
may also correspond to the change in the pulmonary crackles strength as the properties of coating fluids change with the progression of the disease. The connection between the surface properties of the fluids in the lung and the crackle sound generated during the liquid bridge rupture or formation is crucial for understanding the pathology of several respiratory diseases and for improving clinical diagnosis $[34,9]$. However, our model in its present state cannot be directly applied to lung physiology mainly due the topological differences between our study and the real liquid bridge in the airways. In fact, in our model the liquid bridge forms between two planes, while in real airways the bridges block the cylindrical tubes.

We can also notice that the liquid bridge in the airways will form not only due to geometrical contraction of the airways but also due to pressure fluctuations in airflow. It is known from the Bernoulli theory, that the air flow reduces local hydrostatic pressure in proportion to the velocity squared. In a flexible tube, if a given region has a smaller diameter, the local pressure at that point has even lower pressure since the velocity increases inverse proportionally to the crossection area. The lung airways are not only flexible but are also coated with a liquid layer which can easily move to the lower pressure regions generating instabilities and leading to airway closure. In addition, a complex mixture of organic fluids coating the airways may not be treated as a Newtonian fluid. Thus, a direct comparison between the energy released during the rupture of liquid bridges in the lung's airways and the system which we use here is not straightforward. However, our numerical algorithm can be easily implemented in arbitrary geometries, for example, the cylindrical geometry of bronchioles, since it is a standard lattice gas model with low computational cost. The main conclusion of this study is that our hypothesis that the expiratory crackles are generated by liquid bridge formation is a plausible one. Another conclusion is that that numerical model gives accurate results in a situation when it can be tested analytically and thus can be reliably used in more complex situations where analytical solutions are not possible.

\section{Acknowledgments}

This work was supported by Conselho Nacional de Desenvolvimento Científico e Tecnológico (CNPq), Fundação de Amparo a Pesquisa do Estado de São Paulo (FAPESP). Sergey V. Buldyrev acknowledges the partial support 
of this research through the Yeshiva College Bernard Cambson Computational Center.

\section{References}

[1] A. Vyshedskiy, R. M. Alhashem, R. Paciej, M. Ebril, I. Rudman, J. J. Fredberg, R. Murphy, Mechanism of inspiratory and expiratory crackles, Chest 135 (1) (2009) 156-164.

[2] R. H. Notter, Lung Surfactants: basic science and clinical applications, Marcel Dekker, Inc., New York, 2000.

[3] S. P. Arold, B. Suki, A. M. Alencar, K. R. Lutchen, E. P. Ingenito, Variable ventilation induces endogenous surfactant release in normal guinea pigs, Am. J. Physiol. Lung Cell. Mol. Physiol. 285 (2003) L370L375.

[4] R. Skartlien, K. Furtado, E. Sollum, P. Meakin, I. Kralova, Latticeboltzmann simulations of dynamic interfacial tension due to soluble amphiphilic surfactant, Physica A: Statistical Mechanics and its Applications 390 (12) (2011) $2291-2302$.

[5] R. D. Kamm, R. C. Schroter, Is airway-closure caused by a liquid-film instability?, Resp. Physiol. 75 (2) (1989) 141-156.

[6] D. P. Gaver III, R. W. Samsel, J. Solway, Efects of surface tension and viscosity on airway reopening, J. Appl. Physiol. 69 (1) (1990) 74-85.

[7] D. R. Otis, F. Petak, Z. Hantos, J. J. Fredberg, R. D. Kamm, Airway closure and reopening assessed by the alveolar capsule oscillation technique, J. Appl. Physiol. 80 (6) (1996) 2077-2084.

[8] D. P. Gaver III, D. Halpern, O. E. Jensen, J. B. Grotberg, The steady motion of a semi-infinite bubble through a flexible-walled channel, J. Fluid. Mech. 319 (1996) 25-65.

[9] M. Heil, A. L. Hazel, J. A. Smith, The mechanics of airway closure, Respir. Physiol. \& Neurobiology 163 (1-3) (2008) 214 - 221.

[10] A. M. Alencar, S. Arold, S. V. Buldyrev, A. Majumdar, D. Stamenović, H. E. Stanley, B. Suki, Dynamic instabilities in the inflating lung, Nature 417 (2002) 809-811. 
[11] Z. Hantos, J. Tolnai, A. M. Alencar, A. Majumdar, B. Suki, Acoustic evidence of airway opening during recruitment in excised dog lungs, J. Appl. Physiol. 97 (2) (2004) 592-598.

[12] A. M. Alencar, A. Majumdar, Z. Hantos, S. V. Buldyrev, H. E. Stanley, B. Suki, Crackles and instabilities during lung inflation, Physica A 357 (1) (2005) 18-26.

[13] R. T. H. Laënnec, De l'auscultation médiate ou traité du diagnostic de maladies des poumons et du coeur, fondé principalement sur ce nouveau moyen d'exploration, Brosson et Chaudé, Paris, 1819.

[14] R. L. H. Murphy, Jr., S. K. Holford, W. C. Knowler, Visual lung sound characterization by time expanded wave form analysis, The New England J. of Med. 296 (17) (1977) 968-971.

[15] D. Halpern, J. B. Grotberg, Fluid-elastic instabilities of liquid-lined flexible tubes, J. Fluid. Mech. 244 (1992) 615-632.

[16] H. Pasterkamp, S. S. Kraman, G. R. Wodicka, Respiratory sounds, advances beyond the stethoscope, Am. J. Respir. Crit. Care Med. 156 (1997) 974-987.

[17] A. M. Alencar, Z. Hantos, F. Peták, J. Tolnai, T. Asztolos, S. Zapperi, J. S. Andrade, Jr., S. V. Buldyrev, H. E. Stanley, B. Suki, Scaling behavior in crackle sound during lung inflation, Phys. Rev. E 60 (4) (1999) 4659-4663.

[18] A. M. Alencar, S. V. Buldyrev, A. Majumdar, H. E. Stanley, B. Suki, Avalanche dynamics of crackle sound, Phys. Rev. Lett. 87 (2001) 088101.

[19] M. P. Mahajan, M. Tsige, S. Zhang, J. I. Alexander, P. L. Taylor, C. Rosenblatt, Collapse dynamics of liquid bridges investigated by timevarying magnetic levitation, Phys. Rev. Lett. 84 (2) (2000) 338-341.

[20] M. Heil, J. P. White, Airway closure: surface-tension-driven nonaxisymmetric instabilities of liquid-lined elastic rings, J. Fluid. Mech. 462 (2002) 79-109.

[21] D. Langbein, F. Falk, R. Grossbach, Oscillations of liquid columns under microgravity, Microgravity Sciences: Results and Analysis of Recent Spaceflights Advances in Space Research 16 (7) (1995) 23-26. 
[22] A. M. Alencar, E. Wolfe, S. V. Buldyrev, Monte carlo simulation of liquid bridge rupture: Application to lung physiology, Phys. Rev. E 74 (2) (2006) 026311.

[23] J. J. Fredberg, S. K. Holford, Discrete lung sounds: crackles (rales) as stress-relaxation quadrupoles, J. Acoust. Soc. Am. 3 (1983) 1036-1046.

[24] Y. Men, X. Zhang, W. Wang, Capillary liquid bridges in atomic force microscopy: Formation, rupture, and hysteresis, The Journal of Chemical Physics 131 (18) (2009) 184702.

[25] H. Kim, L. C. Saha, J. K. Saha, J. Jang, Molecular simulation of the water meniscus in dip-pen nanolithography, Scanning 32 (1) (2010) 2-8.

[26] R. Heady, J. Cahn, An analysis of the capillary forces in liquid-phase sintering of spherical particles, Metallurgical and Materials Transactions B 1 (1970) 185-189.

[27] R. German, S. Farooq, C. Kipphut, Kinetics of liquid sintering, Materials Science and Engineering: A 105106, Part 1 (0) (1988) 215 - 224.

[28] A. M. Alencar, J. P. Butler, S. M. Mijailovich, Thermodynamic origin of cooperativity in actomyosin interactions: The coupling of short-range interactions with actin bending, Physical Review E 79 (2009) 041906.

[29] L. Sirghi, R. Szoszkiewicz, E. Riedo, Volume of a nanoscale water bridge, Langmuir 22 (3) (2006) 1093-1098.

[30] D. A. Rice, Sound speed in pulmonary parenchyma, J. Appl. Physiol. 54 (1983) 304-308.

[31] A. Leung, S. Sehati, J. D. Young, C. McLeod, Sound transmission between 50 and $600 \mathrm{hz}$ in excised pig lungs filled with air and helium, Journal of Applied Physiology 89 (6) (2000) 2472-2482.

[32] D. F. Ponte, R. Moraes, D. C. Hizume, A. M. Alencar, Characterization of crackles from patients with fibrosis, heart failure and pneumonia, Medical Engineering \& Physics (0) (2012) INPRESS.

[33] W. C. Carter, The forces and behavior of fluids constrained by solids, Acta Metall. 36 (1988) 2283-2292. 
[34] A. M. Alencar, S. V. Buldyrev, A. Majumdar, H. E. Stanley, B. Suki, Relation between crackle sound and the perimeter growth of a cayley tree: Application to lung inflation, Phys. Rev. E 68 (2003) 011909. 


\section{Referências Bibliográficas}

[1] R.M. German. Sintering Theory and Practice. A Wiley-Interscience Publication. Wiley, 1996.

[2] R.M. German, S. Farooq, and C.M. Kipphut. Kinetics of liquid sintering. Materials Science and Engineering: A, 105-106, Part 1(0):215 - 224, 1988.

[3] Ronald Heady and John Cahn. An analysis of the capillary forces in liquid-phase sintering of spherical particles. Metallurgical and Materials Transactions B, 1:185-189, 1970.

[4] Brian A. Hills. An alternative view of the role(s) of surfactant and the alveolar model. Journal of Applied Physiology, 87:1567-1583, 1999.

[5] R.H. Notter. Lung Surfactants: Basic Science and Clinical Applications. Lung Biology in Health and Disease. Marcel Dekker, 2000.

[6] S. P. Arold, B. Suki, A. M. Alencar, K. R. Lutchen, and E. P. Ingenito. Variable ventilation induces endogenous surfactant release in normal guinea pigs. Am. J. Physiol. Lung Cell. Mol. Physiol., 285:L370-L375, 2003. 
[7] Sollum E. Skartlien R., Furtado K. Lattice-boltzmann simulations of dynamic interfacial tension due to soluble amphiphilic surfactant. Physica A - Statistcal mechanics and its application, 390:2291-2302, 2011.

[8] R. D. Kamm and R. C. Schroter. Is airway-closure caused by a liquidfilm instability. Resp. Physiol., 75(2):141-156, 1989.

[9] D. P. Gaver III, R. W. Samsel, and Julian Solway. Efects of surface tension and viscosity on airway reopening. J. Appl. Physiol., 69(1):7485, 1990.

[10] Adriano M. Alencar, Stephen P. Arold, Sergey V. Buldyrev, Arnab Majumdar, Dimitrije Stamenovic, H. Eugene Stanley, and Bela Suki. Physiology: Dynamic instabilities in the inflating lung. Nature, 417(6891):809-811, June 2002.

[11] Zoltan Hantos, József Tolnai, Adriano M. Alencar, Arnab Majumdar, and Béla Suki. Acoustic evidence of airway opening during recruitment in excised dog lungs. J. Appl. Physiol., 97(2):592-598, 2004.

[12] A. M. Alencar, A. Majumdar, Z. Hantos, S. V. Buldyrev, H. E. Stanley, and B. Suki. Crackles and instabilities during lung inflation. Physica A, 357(1):18-26, 2005.

[13] Jeffrey J. Fredberg and Stephen K. Holford. Discrete lung sounds: Crackles (rales) as stress-relaxation quadrupoles. The Journal of the Acoustical Society of America, 73(3):1036-1046, 1983.

[14] Raymond L. H. Murphy, Stephen K. Holford, and William C. Knowler. 
Visual lung-sound characterization by time-expanded wave-form analysis. New England Journal of Medicine, 296(17):968-971, 1977.

[15] Adriano M. Alencar, Zoltán Hantos, Ferenc Peták, József Tolnai, Tibor Asztalos, Stefano Zapperi, José S. Andrade, Sergey V. Buldyrev, H. Eugene Stanley, and Béla Suki. Scaling behavior in crackle sound during lung inflation. Phys. Rev. E, 60:4659-4663, Oct 1999.

[16] Adriano Mesquita Alencar. Crescimento e transporte em estruturas ramificadas. Tese de doutorado, Universidade Federal do Ceará, 1999.

[17] Lucel Sirghi, Robert Szoszkiewicz, and Elisa Riedo. Volume of a nanoscale water bridge. Langmuir, 22(3):1093-1098, 2006.

[18] David L. Malotky and Manoj K. Chaudhury. Investigation of capillary forces using atomic force microscopy. Langmuir, 17(25):7823-7829, 2001.

[19] Brandon L. Weeks, Mark W. Vaughn, and James J. DeYoreo. Direct imaging of meniscus formation in atomic force microscopy using environmental scanning electron microscopy. Langmuir, 21(18):8096-8098, 2005.

[20] T. Thundat, X.-Y. Zheng, G.Y. Chen, and R.J. Warmack. Role of relative humidity in atomic force microscopy imaging. Surface Science, 294(1-2):L939 - L943, 1993.

[21] Mika M. Kohonen and Hugo K. Christenson. Capillary condensation of water between rinsed mica surfaces. Langmuir, 16(18):7285-7288, 2000. 
[22] Leonard R Fisher and Jacob N Israelachvili. Experimental studies on the applicability of the kelvin equation to highly curved concave menisci. Journal of Colloid and Interface Science, 80(2):528 - 541, 1981.

[23] Joonkyung Jang, George C. Schatz, and Mark A. Ratner. Liquid meniscus condensation in dip-pen nanolithography. The Journal of Chemical Physics, 116(9):3875-3886, 2002.

[24] Joonkyung Jang, George C. Schatz, and Mark A. Ratner. Capillary force on a nanoscale tip in dip-pen nanolithography. Phys. Rev. Lett., 90:156104, Apr 2003.

[25] Joonkyung Jang, George C. Schatz, and Mark A. Ratner. How narrow can a meniscus be? Phys. Rev. Lett., 92:085504, Feb 2004.

[26] Joonkyung Jang, George C. Schatz, and Mark A. Ratner. Capillary force in atomic force microscopy. The Journal of Chemical Physics, 120(3):1157-1160, 2004.

[27] Joonkyung Jang, M. A. Ratner, and George C. Schatz. Atomic-scale roughness effect on capillary force in atomic force microscopy. The Journal of Physical Chemistry B, 110(2):659-662, 2006.

[28] Pavel B. Paramonov and Sergei F. Lyuksyutov. Density-functional description of water condensation in proximity of nanoscale asperity. The Journal of Chemical Physics, 123(8):084705, 2005.

[29] Joonkyung Jang, Jonggu Jeon, and Sungu Hwang. Monte carlo study on the water bridge that produces the pull-off force in atomic force 
microscopy. Colloids and Surfaces A: Physicochemical and Engineering Aspects, 300(1-2):60 - 64, 2007.

[30] Yumei Men, Xianren Zhang, and Wenchuan Wang. Capillary liquid bridges in atomic force microscopy: Formation, rupture, and hysteresis. The Journal of Chemical Physics, 131(18):184702, 2009.

[31] Yumei Men, Xianren Zhang, and Wenchuan Wang. Rupture kinetics of liquid bridges during a pulling process: A kinetic density functional theory study. The Journal of Chemical Physics, 134(12):124704, 2011.

[32] H.J. Choi, J.Y. Kim, S.D. Hong, M.Y. Ha, and J. Jang. Molecular simulation of the nanoscale water confined between an atomic force microscope tip and a surface. Molecular Simulation, 35(6):466-472, 2009.

[33] Hyojeong Kim, Leton C. Saha, Joyanta K. Saha, and Joonkyung Jang. Molecular simulation of the water meniscus in dip-pen nanolithography. Scanning, 32(1):2-8, 2010.

[34] Adriano M. Alencar, Elie Wolfe, and Sergey V. Buldyrev. Monte carlo simulation of liquid bridge rupture: Application to lung physiology. Phys. Rev. E, 74:026311, Aug 2006.

[35] P.G. De Gennes, F. Brochard-Wyart, and D. Quéré. Capillarity and Wetting Phenomena: Drops, Bubbles, Pearls, Waves. Springer, 2004.

[36] Drew Myers. Surfaces, Interfaces, and Colloids Principles and Applications. VCH, 1991. 
[37] D.W. Langbein. Capillary surfaces: shape-stability-dynamics, in particular under weightlessness. Springer tracts in modern physics. Springer, 2002.

[38] D Langbein, F Falk, and R Großbach. Oscillations of liquid columns under microgravity. Advances in Space Research, 16(7):23 - 26, 1995.

[39] A.D. Myshkis. Low-gravity fluid mechanics: mathematical theory of capillary phenomena. Springer-Verlag, 1987.

[40] Lianmin Zhou. On stability of a catenoidal liquid bridge. Pac. J. Math, $178,1997$.

[41] Thomas I. Vogel. Stability of a liquid drop trapped between two parallel planes. SIAM Journal on Applied Mathematics, 47(3):pp. 516-525, 1987.

[42] Thomas I. Vogel. Stability of a liquid drop trapped between two parallel planes ii: General contact angles. SIAM Journal on Applied Mathematics, 49(4):pp. 1009-1028, 1989.

[43] W.C. Carter. The forces and behavior of fluids constrained by solids. Acta Metallurgica, 36(8):2283 - 2292, 1988.

[44] David Megias-Alguacil and Ludwig J. Gauckler. Analysis of the capillary forces between two small solid spheres binded by a convex liquid bridge. Powder Technology, 198(2):211 - 218, 2010.

[45] Youchuan Chen, Yongzhi Zhao, Hongli Gao, and Jinyang Zheng. Liquid bridge force between two unequal-sized spheres or a sphere and a plane. Particuology, 9(4):374 - 380, 2011. 
[46] A.H. Nazemi and A. Majnooni-Heris. A mathematical model for the interactions between non-identical rough spheres, liquid bridge and liquid vapor. Journal of Colloid and Interface Science, 369(1):402 - 410, 2012.

[47] Matthias Heil, Andrew L. Hazel, and Jaclyn A. Smith. The mechanics of airway closure. Respiratory Physiology Eamp; Neurobiology, $163(1-3): 214-221,2008$.

[48] Matthias Heil and Joseph P. White. Airway closure: surface-tensiondriven non-axisymmetric instabilities of liquid-lined elastic rings. $J$. Fluid Mech, 462:79-109, 2002.

[49] F. M. Orr, L. E. Scriven, and A. P. Rivas. Pendular rings between solids: meniscus properties and capillary force. Journal of Fluid Mechanics, 67(04):723-742, 1975.

[50] Nicholas Metropolis and S. Ulam. The monte carlo method. Journal of the American Statistical Association, 44(247):335-341, September 1949.

[51] Nicholas Metropolis, Arianna W. Rosenbluth, Marshall N. Rosenbluth, Augusta H. Teller, and Edward Teller. Equation of state calculations by fast computing machines. The Journal of Chemical Physics, 21(6):10871092, 1953.

[52] D.P. Landau and K. Binder. A Guide To Monte Carlo Simulations In Statistical Physics. Cambridge University Press, 2005.

[53] K. Binder and D.W. Heermann. Monte Carlo Simulation in Statisti- 
cal Physics: An Introduction. Springer Series in Solid-State Sciences. Springer, 2010.

[54] D. Frenkel and B. Smit. Understanding Molecular Simulation: From Algorithms to Applications. Computational Science. Academic Press, 2002.

[55] Charles H Bennett. Efficient estimation of free energy differences from monte carlo data. Journal of Computational Physics, 22(2):245 - 268, 1976.

[56] Andrey Vyshedskiy, Ruqayyah M. Alhashem, Rozanne Paciej, Margo Ebril, Inna Rudman, Jeffrey J. Fredberg, and Raymond Murphy. Mechanism of inspiratory and expiratory crackles. CHEST, 135:156-164, 2009.

[57] H.C. Hamaker. The london - van der waals attraction between spherical particles. Physica, 4(10):1058 - 1072, 1937.

[58] D. Myers. Surfaces, interfaces, and colloids: principles and applications. Wiley-VCH, 1999. 